OPEN ACCESS

Edited by:

Sujogya Kumar Panda, KU Leuven, Belgium

Reviewed by: Rahul Kumar Dhaka, Chaudhary Charan Singh Haryana Agricultural University, India Roberto Vazquez Munoz, UCONN Health, United States

*Correspondence: Bismita Nayak nayakb@nitrkl.ac.in

${ }^{t}$ These authors have contributed equally to this work

Specialty section: This article was submitted to Biomedical Nanotechnology, a section of the journal Frontiers in Nanotechnology

Received: 10 July 2021 Accepted: 04 October 2021 Published: 25 October 2021

Citation:

Das P, Ghosh S and Nayak B (2021) Phyto-fabricated Nanoparticles and Their Anti-biofilm Activity: Progress and Current Status.

Front. Nanotechnol. 3:739286. doi: 10.3389/fnano.2021.739286

\section{Phyto-fabricated Nanoparticles and Their Anti-biofilm Activity: Progress and Current Status}

\author{
Puja Das ${ }^{\dagger}$, Sayantan Ghosh ${ }^{\dagger}$ and Bismita Nayak* \\ Immunology and Molecular Medicine Laboratory, Department of Life Science, National Institute of Technology, Rourkela, India
}

Biofilm is the self-synthesized, mucus-like extracellular polymeric matrix that acts as a key virulence factor in various pathogenic microorganisms, thereby posing a serious threat to human health. It has been estimated that around $80 \%$ of hospital-acquired infections are associated with biofilms which are found to be present on both biotic and abiotic surfaces. Antibiotics, the current mainstream treatment strategy for biofilms are often found to be futile in the eradication of these complex structures, and to date, there is no effective therapeutic strategy established against biofilm infections. In this regard, nanotechnology can provide a potential platform for the alleviation of this problem owing to its unique sizedependent properties. Accordingly, various novel strategies are being developed for the synthesis of different types of nanoparticles. Bio-nanotechnology is a division of nanotechnology which is gaining significant attention due to its ability to synthesize nanoparticles of various compositions and sizes using biotic sources. It utilizes the rich biodiversity of various biological components which are biocompatible for the synthesis of nanoparticles. Additionally, the biogenic nanoparticles are eco-friendly, cost-effective, and relatively less toxic when compared to chemically or physically synthesized alternatives. Biogenic synthesis of nanoparticles is a bottom-top methodology in which the nanoparticles are formed due to the presence of biological components (plant extract and microbial enzymes) which act as stabilizing and reducing agents. These biosynthesized nanoparticles exhibit anti-biofilm activity via various mechanisms such as ROS production, inhibiting quorum sensing, inhibiting EPS production, etc. This review will provide an insight into the application of various biogenic sources for nanoparticle synthesis. Furthermore, we have highlighted the potential of phytosynthesized nanoparticles as a promising antibiofilm agent as well as elucidated their antibacterial and antibiofilm mechanism.

Keywords: metal oxide nanoparticles, green synthesis, phyto-synthesis, anti-biofilm activity, biofilm related complications

\section{INTRODUCTION}

The discussion regarding biofilms was first initiated in the 17th century by Antonie Van Leeuwenhoek when he reported the presence of microbial aggregates in the plaque scraped from his teeth (Gebreyohannes et al., 2019). In 1978, Costerton and his fellows coined the term bacterial biofilm and described it as an organized community of microorganisms that remain adhered to a surface and enclosed by an extracellular matrix (Costerton et al., 1978). Numerous bacterial and 
fungal species are known to exhibit two modes of development i.e. free-living/planktonic mode and the sessile, surface-adhered mode within biofilms (Oshiro et al., 2019) (Rumbaugh and Sauer, 2020). Biofilm can be defined as "A microbially derived sessile community characterized by cells that are irreversibly attached to a substratum or interface or to each other are embedded in a matrix of extracellular polymeric substances that they have produced, and exhibit an altered phenotype with respect to growth rate and gene transcription" (Gebreyohannes et al., 2019). The extracellular polymeric substance (EPS) of biofilm is generally composed of cellulose, proteins, nucleic acids, lipids, extracellular DNA, alginates, poly$\mathrm{N}$-acetyl glucosamine, polysaccharides, extracellular teichoic acid, and other organic molecules (Costa et al., 2018). EPS has several crucial functions within the biofilm, like developing physical and social interactions, enhancing the rate of gene transfer, and protection from antimicrobials (Flemming et al., 2016). Biofilm is one of the prominent causes of most chronic infections (Lahiri et al., 2021). The microbes present within the biofilm varies from their planktonic equivalents with respect to phenotypic behavior, antimicrobial resistance, and gene regulations. The central role of biofilm is to safeguard the microorganisms present within it from external hostile factors such as temperature, UV, biocides, desiccation, host's immune system, antibiotics, nutrient deficiency, thereby making them resistive to stressful conditions (Yin et al., 2019). Moreover, biofilms form an essential part of the human body, having both constructive and destructive effects on human health depending upon the microbial species and its localization (Macfarlane and Dillon, 2007). Mucosal microflora can exist in both planktonic as well as in sessile forms within biofilms. They play a crucial role in maintaining homeostasis and act as a bacterial reservoir. Various diseases, including inflammatory bowel diseases, are caused by dysregulation or disturbance in the mucosal biofilm of healthy bacteria (IBD) (Xavier and Podolsky, 2007). Further, a microbial biofilm of the gut helps in water retention and prevents any microbial infection (Dunne, 2002) (von Rosenvinge et al., 2013). Moreover, Lactobacillus biofilms on vaginal or intestinal epithelia may play an essential role in protecting healthy people from sexually transmitted diseases and intestinal or urinary infections (Watters et al., 2016) (Santos et al., 2016) (Emanuel et al., 2010).

Apart from rendering protection to microbial cells, biofilms can implement various tactics to escape the defense mechanism of the host. Microorganisms within a biofilm can survive in anoxic conditions with limited nutrition due to alteration in metabolism, protein synthesis, and gene expression which lead to reduced metabolic activity and a low rate of cell proliferation (Donlan and Costerton, 2002) (Hall-Stoodley and Stoodley, 2009). Furthermore, these changes make the bacteria more resistant to antimicrobial drugs due to the inactivation of the antimicrobial targets or lowering the cellular function that antimicrobials tend to obstruct. Neither innate nor active immune responses are capable to eradicate the biofilm completely, rather it stimulates the process of the collateral damage of tissue. As a consequence, biofilm facilitates the establishment of chronic infections (Vestby et al., 2020).
According to the reports of the U.S. National Institutes of Health, $80 \%$ of all microbial infections within the human body are caused due to biofilms (Khatoon et al., 2018). Microbial biofilms can affect different parts of the body like the respiratory system, reproductive organs, oral cavity, and urinary tract; also, it may develop infections on implanted medical devices (Ramage and Williams, 2013) (Mohammad Reza, 2018). Further, studies have suggested that fungal infections, in particular, caused by the genera Candida, Cryptococcus, and Aspergillus are responsible for more than a million deaths per year (Janbon et al., 2019). With the increased cases of immunocompromised patients and augmented application of medical implants, which forms the potential substrate for biofilm development, people are becoming more susceptible to such infections (Cauda, 2009), (Lebeaux et al., 2014). The bacteria which are commonly associated with biofilmassociated infections include Pseudomonas fluorescens, Pseudomonas aeruginosa, Vibrio cholera, Escherichia coli among Gram-negative bacteria. Among Gram-positive, Staphylococcus aureus, Staphylococcus epidermidis, and enterococci are the common biofilm producers which are the leading cause of nosocomial infections (Barbosa et al., 2016). Apart from providing resistance against the antibiotic, biofilms play an important role in the pathogenicity of different bacteria. For example, in P. aeruginosa, there is upregulation of various virulence factors during biofilm formation (Landini et al., 2010). Apart from the hospital environment, biofilm also affects various other sectors such as water treatment (microbial population of active silt can be destroyed by biofilm), and food manufacturing industries (biofilm formation by spoilage and pathogenic microorganism), and textiles industries (Borzenkov et al., 2020).

Antibiofilm approaches which are currently being applied to combat biofilms are the application of biofilm degrading agents, anti-adhesion compounds, or disruption of biofilm development at initial stages. Typically, infections transmitted via medical equipment are cured using traditional antimicrobial agents. These standard antimicrobials are generally susceptible to planktonic cells but often fail to treat sessile counterparts within the biofilm, thereby necessitates surgical elimination of implants (Nadell et al., 2009). Moreover, the application of disinfectants and sanitizers is ineffective against biofilm (Sharahi et al., 2019) (Malhotra et al., 2015). Several approaches, such as maintaining appropriate hygiene and applying antibiotic prophylaxis, are commonly used to manage and prevent biofilm development on medical equipment. Further, incorporation of antimicrobial agents (antiseptics, antibiotics, or metals) on the surface or development of extremely smooth surfaces are some of the techniques to develop biofilm resistive medical equipment (Camargo et al., 2009). Most of them could not fulfill the criteria for long-term application. Also, to prevent biofilm development on catheters, a new approach called the Antimicrobial lock technique (ALT) has been established, in which antibacterial agents are incorporated on the catheter surface profusely. But this technique has certain drawbacks like toxicity and the development of secondary infections which obstruct its effective applications (Banerjee et al., 2020).

Antibiotics are the primary approach to treat a bacterial infection, but due to the rapid increase in bacterial resistance 
against antibiotics, the search for new tools has become a hot research topic worldwide. The conventional therapy for treating bacterial biofilm includes the cocktail of different antibiotics which has various killing mechanisms. However, with the increase in resistance against the antibiotic, traditional treatments are collapsing in their efforts to combat biofilm. Likewise, the prime anti-mycotics which are used to treat fungal infections include azoles (e.g., fluconazole), polyenes (e.g., amphotericin B), echinocandins (e.g., caspofungin), allylamines (e.g., terbinafine) (Tits et al., 2020); among these, only liposomal formulations of amphotericin $\mathrm{B}$ and echinocandins can be used to treat biofilm-based infections (Kuhn et al., 2002) (Uppuluri et al., 2011). Further, the EPS matrix inhibits the penetration of antibiotics within the biofilm (Pinto et al., 2020). Also, the modifications in the microhabitat within biofilm lead to a change in nutrition supply, development of anoxic conditions, reduced water accessibility, temperature modification. As a result, adaptive stress responses in bacterial cells are enhanced. Consequently, the bacterial cells transform into persisters, which are highly protected spore-like structures, which promotes the emergence of drug resistance (Lahiri et al., 2021).

With the failure of the traditional approach to eliminate biofilm, it is now imperative to explore the possibilities of new drugs and approaches to combat biofilm. The application of nanotechnology in the field of medicine has shown promising results in recent years due to its multidisciplinary approach thereby giving rise to a new arena called "nanomedicine." Lately, The use of various nanoparticles is emerging as a potentially promising alternative to antibiotics for combatting and treating biofilm-producing pathogens, as nanoparticles follow different mechanisms of action to target, against which the bacteria are unable to develop resistance. Nanoparticles impart antibiofilm activity via various mechanisms such as generation of ROS, EPS destruction, inhibition of quorum sensing, etc (Baptista et al., 2018). Even though numerous outstanding research has been published in this area, to the best of our knowledge, no comprehensive review of the current developments has been published yet. To this end, this literature reviews the various biogenic approaches employed for the fabrication of nanoparticles. Herein, this review offers an update on the phyto-synthesized nanoparticles and their role in combatting microbial biofilms. This survey might open the way for further progress in this area and we can expect that the application of phyto-synthesized nanomaterials will expand the spectrum of new possibilities for metal oxide nanomaterials and widen the research domains of combating harmful bacteria and biofilms.

\section{DEVELOPMENT OF BIOFILM}

The event of biofilm formation is a multistep mechanism involving series of biological, chemical, and physical changes depending upon different external stimuli such as extreme $\mathrm{pH}$, excessive temperature, high pressure, increased salt concentration, desiccation, UV radiation, limited nutrition, and antimicrobial agents (Galié et al., 2018). The formation of biofilm takes place with a reversible adhesion of microbial cells to the substratum, followed by the irreversible attachment, which is facilitated by adhesive components of bacteria and short-range interactions. This process of attachment is progressed further with the secretion of EPS. Subsequently, the bacterial cells develop into a systematized structure enclosed within the EPS matrix. The bacterial cells can leak out from the mature biofilm and get disperse into the environment and colonize new sites. This overall event can be classified into five different phases, viz. 1) reversible attachment 2) Irreversible adhesion 3) microcolonies formation 4) biofilm maturation, and 5) dissemination (Figure 1; Kirmusaoglu, 2016).

Typically, the reversible attachment is initiated with adherence of microbial cells to the preconditioned substrate in a way that bacteria continue to be in 2D-Brownian motion and can get easily separated from the surface either due to bacterial motion or shearing effects of a fluid flowing over the surface. This initial attachment is aided by various interactions like hydrophobic forces, electrostatic forces, Lifshitz-van der Waals interactions, and microbial cell surface appendages like flagella, fimbriae, pili, and curli-fibers. The second stage is characterized by immobilization and irreversible attachment of microbial cells and thereby monolayer formation of microbial cells due to the involvement of several interactions like hydrophobic interactions, dipole-dipole interactions, hydrogen, covalent and ionic bonding. Subsequently, in the third stage, irreversible adhesion is proceeded by EPSs secretion which forms the vital component of biofilm's extracellular matrix. Further, the development of microcolonies via rapid cell proliferation characterizes this phase. EPS plays vital roles in biofilm development, surface attachment, water retention, nutrition entrapment, exchange of genetic material, and protection of microbial cells. As the width of biofilm increases, the fourth stage is reached i.e. maturation which is facilitated by quorum sensing. This stage is characterized by the development of an intricate $3 \mathrm{D}$ structure with water-filled channels which circulate nutrients to different cells and remove unwanted waste material. The terminal step of biofilm development is the detachment process or the dispersal process. In this stage, due to the dynamic instability of the biofilm matrix, the microbial cells detach either passively or actively and enter into the surroundings as planktonic cells. These released cells can also propagate to another surface where they may get adsorb and develop new environmental niches. The detachment process is aided by the secretion of numerous saccharolytic enzymes which facilitate the release of microbes from the EPS surface. For example, alginate lyase secreted by Pseudomonas aeruginosa and Pseudomonas fluorescens, N-acetyl-heparosan lyase secreted by Escherichia coli, hyaluronidase secreted by Streptococcus equi, causes lysis of EPS and thereby facilitate detachment (Renner and Weibel, 2011) (Ramasamy and Lee, 2016) (Kirmusaoglu, 2016) (Muhammad et al., 2020).

\section{INFECTIONS ASSOCIATED WITH BIOFILM}

With the ability to grow on both living and non-living surfaces, biofilm forms one of the primary causes of chronic as well as 


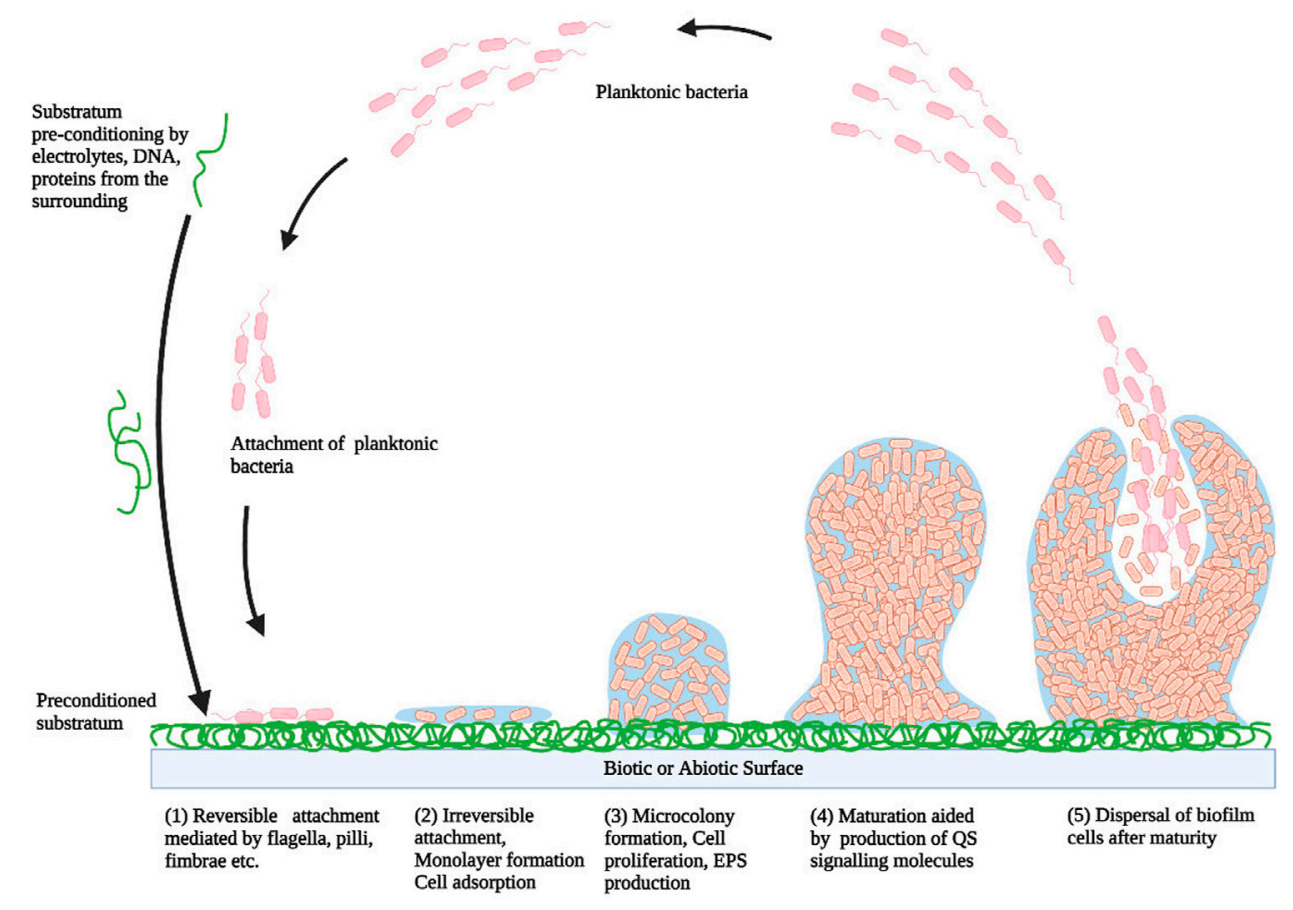

FIGURE 1 | A model showing the typical step-wise development of biofilm (1) Reversible attachment, (2) Irreversible attachment (3) Microcolonies formation (4) Biofilm maturation (5) Dissemination (Kirmusaoglu, 2016).

hospital-acquired infections. These include both, device- and non-device-associated infections (Lewis, 2001).

\section{Device-Associated Infections}

Biofilms are generally found to be present on or inside indwelling therapeutic devices like mechanical heart valves, central venous catheters, prosthetic joints, peritoneal dialysis catheters, contact lenses, pacemakers, voice prostheses, and urinary catheters (Donlan, 2001). Its microbial composition depends upon the type and the duration of action of residing devices (Donlan, 2002).

Microbial cells tend to adhere to both soft and hard varieties of contact lenses. The extent of adherence depends on various criteria vis. the nature of substrate, electrolyte concentration, water content, bacterial strain involved, and the material used in contact lenses. The microorganism commonly affecting contact lenses includes E. coli, Staphyloccocus aureus, Pseudomonas aeruginosa, Staphylococcus epidermidis, Proteus, Serratia, Candida species, and so forth (Donlan and Costerton, 2002). Prosthetic valve endocarditis is another biofilm-related complication in which biofilms are developed on mechanical heart valves and nearby organs (Mrsic et al., 2018). A wide range of microorganisms which include Staphylococcus epidermidis, Staphylococcus aureus, Acinetobacter baumannii, Pseudomonas aeruginosa, Klebsiella pneumoniae, Propionibacterium acnes, Enterococcus, Escherichia coli, Candida species, and yeasts, is known to infect different cardiac implants such as pacemakers, defibrillator, prosthetic valves, coronary artery bypass grafts, which gradually forms denser biofilms in vivo as compared to in vitro (Viola and Darouiche, 2011). These cardiac devices associated with biofilms lowers the rate of blood flow and promote hematogenous spread thereby infecting and developing biofilms in other organs (Bosio et al., 2012). Urinary catheters which are generally constructed of latex or silicone are utilized to accumulate urine during operation, prevent urine retention, and measure urine generation, in ICU. These catheters are generally administered up to the urinary bladder via the urethra. Bacterial contamination due to periurethral skin colonization results in bladder migration of the microbial cells thereby leading to biofilms establishment on these catheters (Kokare et al., 2009). Pseudomonas aeruginosa, Klebsiella pneumoniae, Enterococcus faecalis, Streptococcus epidermidis, Proteus mirabilis, and other Gram (-ve) bacteria are among the microorganisms that develop biofilms on these devices (Pelling et al., 2019). Further, these microbes develop an alkaline condition by increasing the $\mathrm{pH}$ which facilitates the formations of struvite biofilms inside these catheters (Neethirajan et al., 2014). Moreover, Biofilm formation on central venous catheters is quite frequent, although the location and intensity of biofilm formation are both dependent on the duration of action of these catheters. Patients with bone marrow transplants are at higher risk of biofilm infections as they require long-term vascular catheters. The development of the bacterial community depends on the fluid composition which is being administered through these catheters. For instance, grampositive bacteria like Staphylococcus epidermidis and Staphylococcus aureus, show poor development in the intravenous fluids, while gram-negative aquatic bacteria, such 
as Klebsiella sp., Enterobacter sp., and P. aeruginosa, can grow well in such liquids (Raad et al., 1993) (Jamal et al., 2018). Many research reports have indicated that biofilm infections have been related to aseptic loosening of joint prostheses. Further, infections in prosthetic joints caused by Propionibacterium acnes or Staphylococcus epidermidis can lead to serious problems and a high death rate, post joint replacement operation (Del Pozo and Patel, 2009). Moreover, numerous studies have reported that microorganisms like Staphylococcus aureus, Bacillus species, Klebsiella pneumoniae, Escherichia coli, Pseudomonas aeruginosa, etc. are found to contaminate and develop biofilms in endotracheal tubes (Vandecandelaere and Coenye, 2015). Also, different bacteria of breast ducts and tissue lead to biofilm development on breast implants (Pajkos et al., 2003).

\section{Non-Device Associated Infections}

Most of the chronic infections are related to biofilms, as microbial cells present within the biofilms are resistive to the host defense system, antibiotics, and other therapies. Some of the non-devicerelated bacterial infections are periodontitis, osteomyelitis, cystic fibrosis, otitis media, and chronic wounds (Vestby et al., 2020).

Periodontitis is the infection of gums, generally caused due to poor oral health. Injury of soft tissues, damage to the bones supporting the teeth, and occasional tooth loss are the common characteristics of this infection (Guiglia et al., 2010). It is caused by biofilm-forming bacteria such as $P$. aerobicus and Fusobacterium nucleatum which colonize the teeth surface followed by mucosal cell invasion (Jamal et al., 2018). They may also alter the calcium flow within epithelial cells as well as release toxins. This may lead to the development of plaque within few weeks which can be mineralized with phosphate and calcium ions, thereby leading to the development of calculus or tartar (Overman, 2000). Also, Candida albicans are known to form biofilms on the mucosal layer of the oral cavity. And the synergistic relationship between C. albicans and Streptococcus mutans within the biofilm of oral plaque facilitates bacterial colonization and thereby promotes the formation of dental caries (Ponde et al., 2021). In addition, Osteomyelitis is the disease of bones caused by fungal or bacterial cells. Bacteria invade the bone via blood vessels, injury, or prior infections, thereby causing infection in the bone's metaphysis. This leads to infiltration of WBCs at the site of infection which attacks the invading bacteria via phagocytosis or secretion of lytic enzymes thereby resulting in the formation of pus and further spread through bone blood vessels. This leads to blockage of proper blood flow and thereby tissue damage in the infected site of the bone (Goodrich, 2019). Cystic fibrosis is one of the most studied biofilm-associated infections which primarily affects the respiratory and the digestive system and is characterized by the generation of viscous mucus and chronic infections (Ciofu et al., 2015). In earlier stages, the airways are primarily colonized by Staphylococcus aureus and Haemophilus influenzae, while at later stages Pseudomonas aeruginosa dominates (Hector et al., 2016). Large numbers of polymorphonuclear leukocytes (PMNs) are being recruited to the infected site in response to the presence of biofilm, thereby causing persistent inflammation, airway blockage, loss of lung function, and tissue damage. Further, the anaerobic condition developed due to the metabolic activity of bacteria facilitates the biofilm mode of $P$. aeruginosa even more (Rada, 2017). Otitis media (OM) is a condition in which the middle ear chamber gets inflamed, commonly affecting pre-school-aged children (DeAntonio et al., 2016). OM can further be classified into chronic supportive OM (CSOM), OM with effusion (OME), and acute OM (AOM) (Schilder et al., 2017). Generally, biofilm develops in the middle-ear mucosa and middle-ear fluid in case of chronic otitis media (COM) patients. The microbial community consisting of Escherichia coli, Haemophilus influenzae, Staphylococcus aureus, Pseudomonas aeruginosa, and Moraxella catarrhalis as well as other pathogenic bacteria is responsible for the biofilm formation (Homøe et al., 2009). Any damage to living tissue is generally referred to as wounds which can be caused due to various reasons like burns, abrasions, cuts, and surgery or due to other underlying conditions like diabetes (Fijan et al., 2019). Recent studies have indicated that the biofilm mode of bacterial growth is the prime cause of chronic wound infections. Chronic wounds are generally colonized by several bacterial communities among which Staphylococcus aureus is found in majority (Brackman et al., 2013). Aerobic bacteria such as Staphylococcus aureus, Staphylococcus epidermidis, and Pseudomonas aeruginosa are generally isolated from the surface of chronic wounds whereas anaerobic bacteria such as Bacteroides sp., Clostridium sp., Peptostreptococcus sp., and Fusobacterium sp. are generally found in deeper tissue (Percival et al., 2012).

\section{DIFFERENT METHODS OF NANOPARTICLES SYNTHESIS}

In the last decade, nanoparticle research activities grew dramatically, with a primary focus on the synthesis of nanoparticles. The synthesis of nanoparticles is important to understand the particulate formation process, fine-tune the physicochemical characteristics of the nanoparticles, and enable specific functionalities and applications (Jeevanandam et al., 2016). For the synthesis of nanoparticles, a variety of physical, chemical, and biological techniques are available. These three methods can further be classified into two categories: 1) the top-bottom approach and 2) the bottom-top approach (Yadi et al., 2018) (Figure 2).

In top-bottom approaches, the bulk counterpart of the compound is broken down systematically thereby leading to the synthesis of fine nanostructures (Dhand et al., 2015). Although this approach is easier to carry out; it is not appropriate to generate informal shaped and very minute particles (Jamkhande et al., 2019). Some of the typically applied top-bottom methods for bulk production of nanoparticles are electron beam lithography, photolithography, thermal/laser ablation, electro explosion, sputtering, anodization, ion and plasma etching, and milling techniques (Ovais et al., 2017). The most significant drawback of the top-bottom method is surface structure imperfections, which impose significant limitations since a material's surface structure has a crucial 


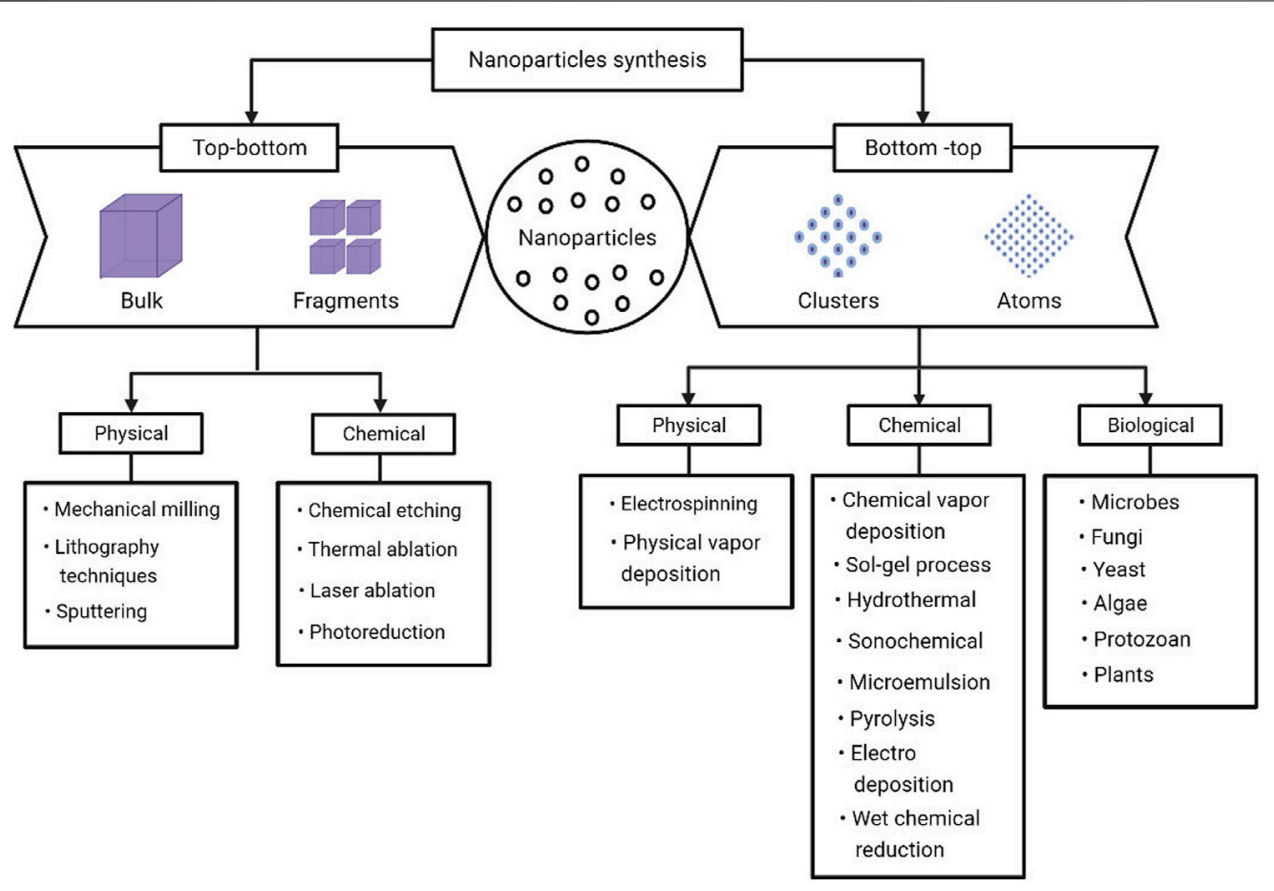

FIGURE 2 | Various approaches available for the synthesis of metal nanoparticles (Patra and Baek, 2014).

role in surface chemistry and the physical characteristics of the material. For example, lithographed nanowires are not necessarily smooth and may have many impurities and structural defects on their surface (Mittal et al., 2013).

The bottom-top approach is an alternative that has the merit to produce less waste and hence is more economical. The synthesis process in bottom-top methods begins with the amalgamation or amassing of atoms and molecules into nuclei, followed by the fabrication of a diverse range of nanoscale particles (Mukherjee et al., 2001). Self-assembly of monomer/polymer molecules, sol-gel processing, chemical vapor deposition (CVD), chemical or electrochemical nanostructural precipitation, laser pyrolysis, plasma or flame spraying synthesis, and biosynthesis are some of the techniques which fall under the bottom-top approach (Daraio and Jin, 2012).

Among the physical, chemical, and biological modes of synthesis, the most effective are the ones that utilize environment-friendly techniques. Even though these nanoparticles have enormous applications in numerous fields, they tend to impart severe toxic effects which nullify the benefits of the material itself. Further, in the field of medicine where sustainability and safety have a vital role to play, the application of green nanotechnology would be more preferred. A green nanotechnology is a multidisciplinary approach that aims to manufacture nanomaterials in an efficient, responsible, and sustainable way thereby holding great potential in biomedicine with special importance on the environment, health, and safety (Kumar et al., 2015). To overcome the issue of toxicity, nanotechnology and green chemistry amalgamate to develop environment-friendly nanoparticles (Lateef et al., 2016). The crucial parameters for the synthesis of nanoparticles are the selection of an eco-friendly solvent, a harmless stabilizing agent, and an effective material for reduction (Jadoun et al., 2021). Biosynthesis is a division of chemistry called "Green Chemistry" which designs and develops chemical components and processes that minimize or exclude the application of hazardous substances (Anastas and Warner, 1998). Biosynthesis fabricates nanoparticles by utilizing the benefits of nature and the environment via non-toxic, clean, ecofriendly green chemistry methods which include the use of organisms such as bacteria, plants, fungi (Duan et al., 2015).

\section{Physical Method}

Physical methods used for the synthesis of nanoparticles include lithography techniques, mechanical milling, sputtering, electrospinning, physical vapor deposition (PVD), and so on (Khodashenas and Ghorbani, 2014). Although physical techniques are relatively quicker and do not require the use of hazardous chemicals, they have certain major limitations like the requirement of expensive equipment and infrastructure, maintenance of high pressure, and temperature (Thakkar et al., 2010).

\section{Chemical Method}

Among chemical methods, the most widely applied methods for nanoparticles synthesis include chemical vapor deposition, solgel process, hydrothermal, sonochemical, wet chemical reduction, microemulsion/colloidal, pyrolysis, chemical etching, thermal ablation, laser ablation, photoreduction, and electrodeposition (Nam and Luong, 2019). The composition and the size of the produced nanoparticles depend on the reducing agents 
employed, reaction time, temperature, and the length of the surfactant molecule (Simeonidis et al., 2007). The use of inorganic and organic salts as reducing agents is one of the most common methods which is frequently employed due to simple procedures and minimal equipment requirements (Vijayaraghavan and Ashokkumar, 2017). The incorporation of hazardous chemicals in the synthesis, as well as the disposal of these reagents, is a key drawback of chemical techniques (Khodashenas and Ghorbani, 2014).

\section{Biological Method}

The biological method employs the use of various biological agents for the synthesis of nanoparticles, such as bacteria, fungus, yeast, virus, protozoan, microalgae, macroalgae, and plant biomass/extract (Shah et al., 2015) (Singh et al., 2016c). Although the biological method takes a longer time to produce nanoparticles compared to chemical and physical methods; the biological synthesis of nanoparticles has the benefits of low toxicity, cost-effectiveness, quick synthesis, ease of scaling up, and simple synthesis procedure (Sharma et al., 2019). One of the prime advantages of plant-mediated biosynthesis is that no hazardous residue products are left on the particles. When people consume them directly, such as through creams or clothing, even minute quantities of hazardous residues can make their safe application unfeasible (Sau and Rogach, 2010) (Fakhari et al., 2019) (Bhattacharya et al., 2019). Also, chemical and physical methods of nanoparticles synthesis are exorbitant due to the fixed price of reagents and apparatus. Moreover, the enormous amount of secondary products produced offers a safe disposal issue (Mirzaei and Darroudi, 2017). Unlike microorganism-assisted nanoparticle synthesis, phyto-synthesis procedures do not involve isolation of microorganisms, identification, optimization of growth conditions, culture preparation, and preservation, which are all complicated and tiresome processes (Patil and Kim, 2017). Rather, plant-assisted green synthesis is quick and straightforward, thereby offers a shorter production time, which indicates potential for scaling-up (Shankar et al., 2004) (Song and Kim, 2009) (Salunke et al., 2014). Due to these advantages, numerous research work has been done to explore the potential of biological materials for the production of metallic nanoparticles. These biologically synthesized nanomaterials have the potential to be applied in various fields like therapeutics, diagnostics, surgical nanodevice development, and commercial product production (Kuppusamy et al., 2015). Among the biological approaches of nanoparticles synthesis, the methods based on plants and microbes have been extensively reported in the literature (Gardea-Torresdey et al., 2003) (Kaler et al., 2011) (Dhillon et al., 2012) (Singh et al., 2016c). Microbial synthesis of nanoparticles is, of course, easily scalable, ecologically friendly, and compatible with the product's usage in medicinal applications, but is often more expensive than nanoparticles synthesis from plant extract (Mittal et al., 2013).

\section{Synthesis of Nanoparticles Using Microorganisms}

Although the employment of microorganisms to synthesize nanoparticles is a modern technique; the interaction between metallic ions and microorganisms has been known for years such as the ability of microbial cells to extract and accumulate metals in bioleaching and bioaccumulation procedures (Lombardi and Garcia, 1999). Microorganisms are significant nano factories with enormous potential as they can store and detoxify heavy metals due to a variety of peptides, proteins, organic materials, reducing cofactors, and reductase enzymes that not only convert metallic ions to metal nanoparticles but also provide a natural capping to the synthesized nanoparticles and thereby improving its polydispersity and stability (Singh et al., 2016c). Further, the microorganism-assisted synthesis of nanoparticles is an environment-friendly and cost-effective approach, avoiding the use of harmful, hazardous chemicals as well as the high energy demands of physiochemical synthesis.

Microorganisms can synthesize nanoparticles both intra- and extracellularly via various mechanisms (Mandal et al., 2006). The intracellular synthesis of nanoparticles initiates with electrostatic interaction of the positively charged metallic ions with negatively charged cell wall followed by the entry of the metal ion inside the cell via a special ion transporter. The ions then undergo enzymatic reduction thereby leading to the formation of nanoparticles, which are then dispersed out through the cell wall (Hulkoti and Taranath, 2014). While the extracellular nanoparticle synthesis involves the accumulation of metallic ions on the cell surface followed by its reduction to nanoparticles in the presence of microbial enzymes. The extracellular synthesis is way more advantageous as compared to intracellular methodologies as it eliminates the downstream processing steps which include multiple centrifugation and washing steps, sonication, and so on which make the intracellular synthesis time-consuming and cumbersome (Zhang et al., 2011).

Bacteria are unicellular living creatures that belong to the prokaryotes family and are found in soil, water, and as symbionts in other species (Lemfack et al., 2020). The following genus includes some of the most significant bacteria investigated for the biosynthesis of nanoparticles: Bacillus (Saravanan et al., 2011), Pseudomonas (Husseiny et al., 2007), Klebsiella (Fesharaki et al., 2010), Escherichia (Gurunathan et al., 2009), Enterobacter (Karthik and Radha, 2012), Aeromonas (Jayaseelan et al., 2013), Corynebacterium (Gowramma et al., 2015), Lactobacillus (Mohammed et al., 2021), Weissella (İspirli et al., 2021), Rhodobacter (Bai et al., 2011), Rhodococcus (Otari et al., 2012), Brevibacterium (Kalishwaralal et al., 2010b), Bhargavaea (Singh et al., 2015), Streptomyces (Bukhari et al., 2021), Desulfovibrio (Gong et al., 2018), Shewanella (Kimber et al., 2018), Rhodopseudomonas (He et al., 2007), Pediococcus (Moodley et al., 2018) and others.

Fungi are easy to culture and pose higher bioaccumulation capability thereby forms an efficient, low-cost with simple downstream processing approach for nanoparticles biosynthesis (Pal et al., 2019). Three probable methods have been proposed to elucidate the mycosynthesis of metal nanostructures i.e. electron shuttle quinones, nitrate reduction action, or both (Gahlawat and Choudhury, 2019). Some of the most important fungi studied for the biosynthesis of nanoparticles belong to the following genera: Fusarium (Shafiq et al., 2016), Aspergillus (Jain et al., 2011), Neurospora (Castro- 
Longoria et al., 2011), Mucor (Sathishkumar et al., 2015), Pestalotia (Raheman et al., 2011), Hypocrea (Bhimba et al., 2011), Trichoderma (Prameela Devi et al., 2013), Colletotrichum (Suryavanshi et al., 2017), Lecanicillium (Namasivayam and Chitrakala, 2011), Rhizopus (AbdelRahim et al., 2017), and others. Yeast, which belongs to the fungus class Ascomycetes, has shown promising potential for the production of nanoparticles. Yeasts, like other microbes, have been extensively studied for large-scale extracellular nanoparticle production with simple downstream processing. Yarrowia (Agnihotri et al., 2009), Rhodosporidium (Seshadri et al., 2011), Candida (Rahimi et al., 2016), Saccharomyces (Korbekandi et al., 2016), Rhodotorula (Cunha et al., 2018), etc. are the genera that include some of the most studied yeast for nanoparticles biosynthesis.

Further, algae are excellent candidates for nanoparticles production since they can accumulate metals and reduce metal ions. They also offer several benefits, like low-temperature nanoparticles synthesis, ease of handling, and low toxicity (Priyadarshini et al., 2019). Algae can generate nanoparticles from different metal salts by using enzymes and functional groups found in their cell walls; even edible algae can make metallic nanoparticles ( $\mathrm{H}$ Madkour, 2017). The following genera contain some of the most significant algae investigated for nanoparticle biosyntheses such as Ulva (Ishwarya et al., 2018), Sargassum (Azizi et al., 2014), Caulerpa (Kathiraven et al., 2015), Spirulina (Kalabegishvili et al., 2012), Galaxaura (Abdel-Raouf et al., 2017), Sargassum (Kumar et al., 2012), Padina (Bhuyar et al., 2020), Chlorella (Arsiya et al., 2017), Scendesmus (Aziz et al., 2014), Chlorococcum (Jena et al., 2013), Bifurcaria (Abboud et al., 2014), Gracilaria (de Aragão et al., 2019), Turbinaria (Rajeshkumar et al., 2013), and others.

The protozoan-mediated biosynthesis of nanoparticles has been least studied. Tetrahymena (Mortimer et al., 2011) and Leishmania (Ramezani et al., 2012) are the two genera that have been studied extensively for the biosynthesis of nanoparticles.

Microorganisms mediated synthesis of nanoparticles can occur via two methods i.e extracellular or intracellular. In the case of extracellular synthesis, the microbial cells are cultured in a rotating shaker under optimal conditions for 1-2 days, followed by centrifugation for the removal of biomass. The supernatant obtained is then again incubated with a filter-sterilized metal salt solution which leads to the synthesis of nanoparticles which is indicated by a change in color of the reaction mixture. Subsequently, the reaction mixture is centrifuged at high speed, washed thoroughly several times with water or solvent (ethanol/methanol) and the synthesized nanoparticles are collected as a pellet. In the intracellular synthesis, the microbial culture is incubated for an optimum amount of growth period, followed by its centrifugation and washing with sterile water. The microbial mass is again incubated in a water medium along with the filter-sterilized solution of metal salt. Alike extracellular synthesis, the synthesis of nanoparticles is monitored by a change in color of the reaction mixture. Followed by the incubation period, the nanoparticles are obtained by removal of the biomass with the help of repeated ultrasonication, subsequent washing, and centrifugation which promotes cell wall disruption and thereby a collection of the released nanoparticles (Singh et al., 2016c). However, most microorganism-based nanoparticles synthesis is slow and lowproductive, and nanoparticle recovery involves tedious downstream processing. Additionally, limitations with microorganism-based nanoparticle production include difficult procedures including microbiological samples isolation, culture, and maintenance.

\section{Synthesis of Nanoparticles Using Plants}

Phytonanotechnology, which employs plant-based compounds for the synthesis of nanoparticles, has recently opened up new opportunities in the field of biomedicine, attributed to its superior biocompatibility, scalability, and simple synthesizing method (Noruzi, 2015). Plants can be used either in their live or dead/ inactive form for nanoparticle biosynthesis. Different plants are recognized for their ability to collect metals, which are then reduced to nanoparticles within the cell (Kuppusamy et al., 2016). Numerous research work has been done utilizing different parts of plants as a reductant for nanoparticles synthesis such as leaf, stem, fruit, latex, root, flower, seed, and seed coat (Bhati-Kushwaha and Malik, 2013). The reduction of metal ions into nanoparticles is aided by different plant biomolecules that include organic acids, proteins, vitamins, amino acids as well as secondary metabolites like flavones, ketones, alkaloids, terpenoids, phenolics, saponins, aldehydes, tannins, and polysaccharides. As per numerous reports, these plant-derived metabolites can prevent the agglomeration and aggregation of metallic nanoparticles by reducing and stabilizing the reaction in a non-toxic manner (Nath and Banerjee, 2013) (Duan et al., 2015) (Kuppusamy et al., 2016). Numerous studies have shown that polyphenols derived from plants impart a prominent anti-inflammatory response as well as can be used as potential immunonutrient supplements against inflammatory and autoimmune diseases (Campbell et al., 2019). Carbonyl, methoxide, amino, and hydroxyl are some of the prime functional groups which reduce the metal ions into metallic NPs via electrostatic interaction (Küünal et al., 2018). Phytosynthesised nanoparticles are diverse in shapes and sizes due to variation in concentration and composition of these bioactive molecules among different plants and their consequent interaction with an aqueous solution of metal ions (Li et al., 2011). Plants have several benefits over other biological systems in terms of nanoparticles synthesis: they facilitate largescale nanoparticles production, no requirement of culture maintenance, provide natural capping agents, have a wider array of secondary metabolites, safe to handle, are cheap, and readily accessible (Küünal et al., 2018). The actual mechanism and components that cause plant-mediated synthesized nanoparticles are still unknown (Drummer et al., 2021). Popular plant genera studied extensively for the biosynthesis of nanoparticles are Euphorbia (Elumalai et al., 2010), Ginkgo (Elumalai et al., 2010), Panax (Singh et al., 2016b), Cymbopogon (Ajayi and Afolayan, 2017), Azadirachta (Ahmed et al., 2016), Nigella (Amooaghaie et al., 2015), Cocos (Roopan et al., 2013), Catharanthus (Ahmad et al., 2020), Pistacia (Sadeghi et al., 2015), Nyctanthes (Sundrarajan and Gowri, 2011), Anogeissus (Kora 
et al., 2012), Abutilon (Mata et al., 2015), Pinus (Iravani and Zolfaghari, 2013), Artocarpus (Manik et al., 2020), Citrus (Sujitha and Kannan, 2013), Lawsonia (Naseem and Farrukh, 2015), Gardenia (Karade et al., 2019), Allium (Velsankar et al., 2020), Averrhoa (Isaac et al., 2013), Sinapis (Khatami et al., 2015), Cucurbita (Hu et al., 2019), Santalum (Swamy and Prasad, 2015), Carissa (Joshi et al., 2018), Avena (Amini et al., 2017), Piper (Paulkumar et al., 2014), Onosma (Doğan Çalhan and Gündoğan, 2020), and others.

Briefly, phytosynthesis of nanoparticles is initiated by the addition of plant extracts (root, leaf, flower, or seed) to metal salt solutions in specific ratios. To obtain plant extract, the plant part is properly washed using distilled water to make sure there is no dust or other contaminants, it is then followed by drying and cutting them into small pieces. Subsequently, the pieces are converted into a fine paste using a mixer blender. The paste is diluted using Milli-Q water and heated/boiled for a specific period. Alternatively, the same procedure can be followed using plant extract obtained from dried powdered plant parts. The plant extract is then centrifuged and filtered using filter paper to obtain pure supernatant which is then stored in a refrigerator for further experimental use. Various ratios and concentrations of metal salt solution, the plant extract is used for developing nanoparticles. The reaction mixture is incubated for a longer duration of time and the synthesis of nanoparticles is indicated by the color change of the reaction mixture. Following incubation, the reaction mixture is centrifuged, washed, and collected for further characterization (Singh et al., 2016c) (Dwivedy et al., 2018).

The overall bioreduction process of nanoparticles synthesis utilizing plant extract can be categorized into three stages. The process starts with the activation step characterized by nucleation and reduction of the metal ions. This is followed by the growth phase which involves the coalescence of very tiny particles to form large size nanoparticles with enhanced thermodynamic stability. With the progression of the growth phase, nanoparticles accumulate to form a diverse range of morphologies like spheres, cubes, hexagons, triangles, pentagons, wires, rods, and so on. Finally, in the termination phase, the plant extract stabilizes the nanoparticles and determines its most thermodynamically favorable morphology (Akhtar et al., 2013) (Makarov et al., 2014). The characteristics like quality, morphology, size of the fabricated nanoparticles depend on various parameters like concentration of plant extract, reaction mixture $\mathrm{pH}$, reaction time, metal salt concentration, and temperature (Dwivedi and Gopal, 2010) (Mittal et al., 2013).

\section{PLANT SYNTHESIZED NANOPARTICLES AGAINST BIOFILMS}

Different plants and their parts have been exploited for the phytogenic synthesis of various metal/metal oxide nanoparticles. These phytofabricated nanoparticles have shown excellent antibacterial properties which have paved the way for future research work assessing its antibiofilm properties. Regarding this, various phytosynthesized nanoparticles (Ag,
$\mathrm{Au}, \mathrm{ZnO}, \mathrm{CeO}_{2}, \mathrm{Fe}_{2} \mathrm{O}_{3}, \mathrm{TiO}_{2}, \mathrm{CuO}, \mathrm{Pd}, \mathrm{Se}, \mathrm{NiO}$ ) have been evaluated for their antibiofilm potential which is discussed below (Table 1). Table 2 represents the list of microbially synthesized nanoparticles exhibiting anti-biofilm activity for comparative analysis.

\section{Silver Nanoparticles}

Silver nanoparticles are the most widely studied nanoparticles for their broad-spectrum antimicrobial properties against a wide variety of pathogens. They are known to impart antibacterial properties via several mechanisms such as cell wall and membrane rupture and intracellular biomolecules damage, oxidative stress, etc (Tang and Zheng, 2018). Among various routes employed, plant-mediated synthesis of AgNPs is most preferred due to its several advantages. Numerous research has been carried out using different plants and their parts to study the antibiofilm properties of phytosynthesised AgNPs.

Regarding this, Malaikozhundan et al. have synthesized silver nanoparticles using Momordica charantia fruit extracts (McAgNPs) and evaluated their antibiofilm efficacy against Aeromonas hydrophila and Enterococcus faecalis. Light microscopy and CLSM observations revealed that Mc-AgNPs exhibited significant biofilm inhibitory properties $(100 \mu \mathrm{g} / \mathrm{ml})$ against $E$. faecalis when compared to that of $A$. hydrophila. Further, the application of Mc-AgNPs reduced the hydrophobicity index by $76 \%$ and $88 \%$ in A. hydrophila and E. faecalis respectively as compared to untreated bacteria (Malaikozhundan et al., 2016).

A similar study done by Arya et al. indicated that AgNPs prepared using aqueous leaf extract of Acacia nilotica inhibited biofilm formation by $70 \%$ and $53 \%$ in Pseudomonas aeruginosa and Staphylococcus aureus respectively at a concentration of $0.25 \mu \mathrm{g}$ (Arya et al., 2018).

Accordingly, Choi et al. screened the antibiofilm and antifungal properties of phytofabricated silver nanoparticles (AgNPs) synthesized using Lycopersicon esculentum extracts against Candida species. SEM analysis revealed the biofilm inhibitory effect of the fabricated AgNPs. They suggested that binding of $\mathrm{Ag}$ ions to cell surface resulted in membrane abnormalities, reduced biofilm development, and inhibited growth (Choi et al., 2019). Further, it has been wellestablished that quorum sensing plays a pivotal role in the development of biofilm. Several studies have reported inhibition of biofilm development via anti-QS agents. Regarding this, Ravindran et al. assessed the potential of Vetiveria zizanioides root extract-mediated silver nanoparticles (VzAgNPs) as a promising antibiofilm and anti-QS agent against S. marcescens. The results revealed that VzAgNPs inhibited the production of various QS-mediated virulence factors such as protease, swarming motility, lipase, prodigiosin, EPS productions, and biofilm formation in S. marcescens, thereby reducing its pathogenicity. Further, the genomic studies supported that VzAgNPs target and remarkably inhibit the expression of genes involved in virulence such as $\mathrm{flhD}$, fimC, and bsbB (Ravindran et al., 2018).

Accordingly, Shah et al. evaluated the anti-QS property of Piper betle leaf extract $(\mathrm{Pb})$ mediated AgNPs (Pb-AgNPs) 
TABLE 1 | List of phytosynthesized nanoparticles exhibiting antibiofilm activity

\begin{tabular}{|c|c|c|c|c|c|c|}
\hline Nanoparticles & Plants source & Plant tissue & Size $(n m)$ & $\begin{array}{l}\text { Concentration for } \\
\text { anti-biofilm } \\
\text { activity }\end{array}$ & $\begin{array}{l}\text { Biofilm producing } \\
\text { microorganisms }\end{array}$ & References \\
\hline Ag NPs & Momordica charantia & Fruit extracts & 16 & $100 \mu \mathrm{g} / \mathrm{ml}$ & $\begin{array}{l}\text { Enterococcus faecalis and Aeromonas } \\
\text { hydrophila }\end{array}$ & $\begin{array}{l}\text { Malaikozhundan } \\
\text { et al. (2016) }\end{array}$ \\
\hline Ag NPs & Mimusops elengi & Fruit extract & 10 & $0.125 \mathrm{mg} / \mathrm{ml}$ & Escherichia coli & $\begin{array}{l}\text { Korkmaz et al. } \\
(2020)\end{array}$ \\
\hline GO-Ag NPs & Lagerstroemia speciosa & Floral extract & $60-100$ & 24 and $12 \mu \mathrm{g} / \mathrm{ml}$ & $\begin{array}{l}\text { Enterobacter cloacae and } \\
\text { Streptococcus mutans }\end{array}$ & $\begin{array}{l}\text { Kulshrestha et al. } \\
(2017)\end{array}$ \\
\hline Ag NPs & Punica granatum & $\begin{array}{l}\text { Fruit fleshy } \\
\text { pericarp }\end{array}$ & $5-30$ & - & $P$. aeruginosa & $\begin{array}{l}\text { Govindappa et al. } \\
\text { (2021) }\end{array}$ \\
\hline Ag NPs & Rhododendron ponticum & Leaf extracts & $12-19$ & $1.0 \mathrm{mg} / \mathrm{ml}$ & Biofilm producing bacteria & Nesrin et al. (2020) \\
\hline Ag NPs & Carum copticum & Seed extract & 21.48 & $\begin{array}{l}10,20 \text {, and } 30 \mu \mathrm{g} / \\
\text { ml respectively }\end{array}$ & $\begin{array}{l}\text { C. violaceum, S. marcescens, and P. } \\
\text { aeruginosa }\end{array}$ & Qais et al. (2020) \\
\hline Ag NPs & Mespilus germanica & Leaf extract & 17.60 & $6.25-100 \mu \mathrm{g} / \mathrm{ml}$ & Klebsiella pneumoniae & $\begin{array}{l}\text { Foroohimanjili et al. } \\
\text { (2020) }\end{array}$ \\
\hline $\mathrm{Ag} N \mathrm{NP}$ & garlic & Clove extract & $10-50$ & $100 \mu \mathrm{g} / \mathrm{ml}$ & $\begin{array}{l}\text { Methicillin-resistant } S \text {. aureus and } P \text {. } \\
\text { aeruginosa }\end{array}$ & $\begin{array}{l}\text { Vijayakumar et al. } \\
\text { (2019) }\end{array}$ \\
\hline Ag NPs & Acacia nilotica & Leaf extract & $10-20$ & $0.25 \mu \mathrm{g} / \mathrm{ml}$ & $P$. aeruginosa and $S$. aureus & Arya et al. (2018) \\
\hline Ag NPs & Moringa oleifera & Leaf extract & 30 & $50 \mu \mathrm{g} / \mathrm{ml}$ & $P$. aeruginosa and S. epidermidis & $\begin{array}{l}\text { Gokul Brindha et al. } \\
(2020)\end{array}$ \\
\hline Ag NPs & Prosopis juliflora & Leaf extract & $10-20$ & - & $\begin{array}{l}\text { Bacillus subtilis and Pseudomonas } \\
\text { aeruginosa }\end{array}$ & Arya et al. (2019) \\
\hline Ag NPs & Psidium guajava & Leaf extract & 60 & $250 \mathrm{mM}$ & S. aureus, E. coli and C. albicans & $\begin{array}{l}\text { Gupta et al. } \\
(2014 b)\end{array}$ \\
\hline Ag NPs & Nigella sativa & Seed extract & 55 & $12.5 \mu \mathrm{g} / \mathrm{ml}$ & $\begin{array}{l}\text { Enterococcus faecalis, E. coli, } \\
\text { Klebsiella pneumoniae, } \\
\text { Staphylococcus aureus, and } \\
\text { Pseudomonas aeruginosa }\end{array}$ & $\begin{array}{l}\text { Almatroudi et al. } \\
(2020)\end{array}$ \\
\hline Ag NPs & Nardostachys jatamansi & $\begin{array}{l}\text { Rhizome } \\
\text { extract }\end{array}$ & $10-15$ & $64 \mu \mathrm{M}$ & $P$. aeruginosa and $S$. aureus & $\begin{array}{l}\text { Muthuraman et al. } \\
\text { (2019) }\end{array}$ \\
\hline Ag NPs & $\begin{array}{l}\text { Semecarpus anacardium, } \\
\text { Glochidion lanceolarium, } \\
\text { and Bridelia retusa }\end{array}$ & Leaf extract & $\begin{array}{l}62.72,93.23 \\
\text { and } 74.56 \\
\text { respectively }\end{array}$ & - & $\begin{array}{l}\text { Pseudomonas aeruginosa, } \\
\text { Escherichia coli, and Staphylococcus } \\
\text { aureus }\end{array}$ & $\begin{array}{l}\text { Mohanta et al. } \\
(2020)\end{array}$ \\
\hline Ag NPs & Cordia dichotoma & Fruit extract & $2-60$ & $100 \mu \mathrm{g} / \mathrm{ml}$ & S. aureus and E. coli & $\begin{array}{l}\text { Bharathi et al. } \\
\text { (2018) }\end{array}$ \\
\hline Ag NPs & Citrus macroptera & Fruit extract & 16 & $260 \mathrm{nM}$ & $\begin{array}{l}\text { Bacillus subtilis and Pseudomonas } \\
\text { aeruginosa }\end{array}$ & $\begin{array}{l}\text { Majumdar et al. } \\
(2020)\end{array}$ \\
\hline Ag NPs & Thymus serpyllum & Leaf extract & 25.2 & $100 \mu \mathrm{g} / \mathrm{ml}$ & S. aureus & $\begin{array}{l}\text { Erci and Torlak, } \\
\text { (2019) }\end{array}$ \\
\hline Ag NPs & Eucalyptus globulus & Leaf extract & $5-25$ & $30 \mu \mathrm{g} / \mathrm{ml}$ & S. aureus and $P$. aeruginosa & Ali et al. (2015) \\
\hline Ag NPs & $\begin{array}{l}\text { Dodonaea viscosa and } \\
\text { Hyptis suoveolens }\end{array}$ & Leaf extract & $40-55$ & $10 \mu \mathrm{g} / \mathrm{ml}$ & Candida spp. & $\begin{array}{l}\text { Muthamil et al. } \\
\text { (2018) }\end{array}$ \\
\hline Ag NPs & Azadirachta indica & Latex & $17.4-40.9$ & $4.12-3.25 \mu \mathrm{g} / \mathrm{ml}$ & Candida tropicalis & Al Aboody (2019) \\
\hline Ag NPs & Rosmarinus officinalis & Leaf extract & 20 & - & Parachlorella kessleri & $\begin{array}{l}\text { Velgosova et al. } \\
(2021)\end{array}$ \\
\hline Ag NPs & Styrax benzoin & Gum & $18.7 \pm 1.2$ & - & E. coli & Du et al. (2016) \\
\hline Ag NPs & Acorus calamus $L$ & Plant extracts & 20 & $350 \mu \mathrm{g} / \mathrm{ml}$ & Helicobacter pylori & Prasad et al. (2019) \\
\hline Ag NPs & Lycopersicon esculentum & Fruit extracts & 108.5 & $8 \mu \mathrm{g} / \mathrm{ml}$ & Candida species & Choi et al. (2019) \\
\hline Ag NPs & Vetiveria zizanioides & Root extract & $20-60$ & $2 \mu \mathrm{g} / \mathrm{ml}$ & Serratia marcescens & $\begin{array}{l}\text { Ravindran et al. } \\
\text { (2018) }\end{array}$ \\
\hline Ag NPs & Piper betle & Leaf extract & - & - & $\begin{array}{l}\text { Chromobacterium violaceum and } P \text {. } \\
\text { aeruginosa }\end{array}$ & Shah et al. (2019) \\
\hline Ag NPs & Syzygium cumini & Leaf extract & $10-20$ & $250 \mu \mathrm{g} / \mathrm{ml}$ & $\begin{array}{l}P . \text { aeruginosa, S. aureus, and } C . \\
\text { albicans }\end{array}$ & Gupta et al. (2014a) \\
\hline Ag NPs & Piper betle & Leaf extract & 156.4 & 16 and $32 \mu \mathrm{g} / \mathrm{ml}$ & S. marcescens and P. mirabilis & $\begin{array}{l}\text { Srinivasan et al. } \\
(2018)\end{array}$ \\
\hline Ag NPs & Curcuma longa & Plant extracts & $20-40$ & - & S. aureus and S. pneumoniae & $\begin{array}{l}\text { Kamble and } \\
\text { Shinde (2018) }\end{array}$ \\
\hline Ag NPs & Lilium lancifolium & Leaf extract & 45 & $25-50 \mu l$ & S. pneumoniae and P.aeruginosa & $\begin{array}{l}\text { Al-Ansari et al. } \\
\text { (2019) }\end{array}$ \\
\hline Ag NPs & Ludwigia octovalvis & Leaf extract & $28-50$ & $100 \mu \mathrm{g} / \mathrm{ml}$ & P. aeruginosa and S. epidermidis & $\begin{array}{l}\text { Sarathi Kannan } \\
\text { et al. (2021) }\end{array}$ \\
\hline Ag NPs & Holarrhena pubescens & Bark extracts & 13.15 & $20-25 \mu \mathrm{g} / \mathrm{ml}$ & P. aeruginosa & Ali et al. (2018) \\
\hline Ag NPs & Artemisia scoparia & Plant extracts & 26.68 & $12.81-8.45 \mu \mathrm{g} / \mathrm{ml}$ & $\begin{array}{l}\text { Methylation-dependent restriction } \\
\text { system (MDRS) strain }\end{array}$ & $\begin{array}{l}\text { Moulavi et al. } \\
\text { (2019) }\end{array}$ \\
\hline
\end{tabular}


TABLE 1 | (Continued) List of phytosynthesized nanoparticles exhibiting antibiofilm activity

\begin{tabular}{|c|c|c|c|c|c|c|}
\hline Nanoparticles & Plants source & Plant tissue & Size (nm) & $\begin{array}{l}\text { Concentration for } \\
\text { anti-biofilm } \\
\text { activity }\end{array}$ & $\begin{array}{l}\text { Biofilm producing } \\
\text { microorganisms }\end{array}$ & References \\
\hline Ag NPs & Zataria multiflora & Aerial parts & 25.5 & $4 \mu \mathrm{g} / \mathrm{ml}$ & S. aureus & $\begin{array}{l}\text { Barabadi et al. } \\
\text { (2021) }\end{array}$ \\
\hline Au NPs & Tinospora cordifolia & Stem extract & 16.1 & $150 \mu \mathrm{g} / \mathrm{ml}$ & Pseudomonas aeruginosa PAO1 & Ali et al. (2020) \\
\hline Au NPs & Nigella sativa & Essential oil & - & - & $\begin{array}{l}\text { Staphylococcus aureus and Vibrio } \\
\text { harveyi }\end{array}$ & Manju et al. (2016) \\
\hline Au NPs & Musa paradisiaca & Peel extract & 50 & $100 \mu \mathrm{g} / \mathrm{ml}$ & Enterococcus faecalis & $\begin{array}{l}\text { Vijayakumar et al. } \\
\text { (2017) }\end{array}$ \\
\hline Au NPs & Capsicum annuum & Fruit extract & 19.97 & - & $\begin{array}{l}\text { Pseudomonas aeruginosa and Serratia } \\
\text { marcescens }\end{array}$ & Qais et al. (2021) \\
\hline Au NPs & Trachyspermum ammi & Seed extract & 16.63 & 16 and $32 \mu \mathrm{g} / \mathrm{ml}$ & S. marcescens and L. monocytogenes & $\begin{array}{l}\text { Perveen et al. } \\
(2021)\end{array}$ \\
\hline $\mathrm{ZnO} \mathrm{NPs}$ & Artemisia & Leaf extract & 17 & - & $P$. aeruginosa & $\begin{array}{l}\text { Galedari and } \\
\text { Teimouri (2020) }\end{array}$ \\
\hline $\mathrm{ZnO} \mathrm{NPs}$ & Plectranthus amboinicus & Leaf extract & $20-50$ & $8-10 \mu \mathrm{g} / \mathrm{ml}$ & $\begin{array}{l}\text { Methicillin-resistant Staphylococcus } \\
\text { aureus }\end{array}$ & $\begin{array}{l}\text { Vijayakumar et al. } \\
(2015)\end{array}$ \\
\hline $\mathrm{ZnO}$ NPs & Veronica multifida & Leaf extract & $10-100$ & $10-50 \mu \mathrm{g} / \mathrm{ml}$ & P. aeruginosa and S. aureus & $\begin{array}{l}\text { Doğan and } \\
\text { Kocabaş (2020) }\end{array}$ \\
\hline $\mathrm{ZnO}$ NPs & Artemisia haussknechtii & Leaf extract & $50-60$ & $100 \mu \mathrm{g} / \mathrm{ml}$ & S. epidermidis and $P$. aeruginosa & Alavi et al. (2019) \\
\hline $\mathrm{ZnO} \mathrm{NPs}$ & Costus igneus & Leaf extract & 26.55 & $75-100 \mu \mathrm{g} / \mathrm{ml}$ & $\begin{array}{l}\text { S. mutans, L. fusiformis, P. vulgaris, } \\
\text { and V. parahaemolyticus }\end{array}$ & $\begin{array}{l}\text { Vinotha et al. } \\
\text { (2019) }\end{array}$ \\
\hline $\mathrm{ZnO}$ NPs & Musa balbisiana & $\begin{array}{l}\text { Pseudostem } \\
\text { extract }\end{array}$ & $45-65$ & $100 \mu \mathrm{g} / \mathrm{ml}$ & $P$. aeruginosa & $\begin{array}{l}\text { Basumatari et al. } \\
(2021)\end{array}$ \\
\hline ZnO NPs & Eucalyptus globulus & Essential oil & 40 & $100 \mu \mathrm{g} / \mathrm{ml}$ & S. aureus and $P$. aeruginosa & Obeizi et al. (2020) \\
\hline $\mathrm{ZnO} \mathrm{NPs}$ & Withania somnifera & Leaf extract & 15.6 & $100 \mu \mathrm{g} / \mathrm{ml}$ & E. faecalis and S. aureus & $\begin{array}{l}\text { Malaikozhundan } \\
\text { et al. (2020) }\end{array}$ \\
\hline $\mathrm{ZnO}$ NPs & Myristica fragrans & Leaf ester & $48.32 \pm 2.5$ & - & $\begin{array}{l}\text { MDR-Escherichia coli (E. coli-336), } \\
\text { Methicillin-resistant Staphylococcus } \\
\text { aureus (MRSA-1) }\end{array}$ & $\begin{array}{l}\text { Cherian et al. } \\
(2019)\end{array}$ \\
\hline ZnO NPs & Ochradenus baccatus & Leaf extract & 50 & - & $\begin{array}{l}\text { Chromobacterium violaceum, } \\
\text { Escherichia coli, P. aeruginosa, } \\
\text { Klebsiella pneumoniae, Serratia } \\
\text { marcescens, and Listeria } \\
\text { monocytogenes }\end{array}$ & $\begin{array}{l}\text { Al-Shabib et al. } \\
(2018)\end{array}$ \\
\hline $\mathrm{ZnO} N P s$ & Nigella sativa & Seed extract & 24 & $128-512 \mu \mathrm{g} / \mathrm{ml}$ & $\begin{array}{l}\text { L. monocytogenes, E. coli, C. } \\
\text { violaceum, and P. aeruginosa PAO1 }\end{array}$ & $\begin{array}{l}\text { Al-Shabib et al. } \\
(2016)\end{array}$ \\
\hline CuO NPs & $\begin{array}{l}\text { Cassia fistula and Meia } \\
\text { azedarach }\end{array}$ & Leaf extract & - & $1 \mu \mathrm{g} / \mathrm{ml}$ & K. pneumoniae and H. pylori & Naseer et al. (2021) \\
\hline Cu NPs & $\begin{array}{l}\text { Cochlospermum } \\
\text { gossypium }\end{array}$ & $\begin{array}{l}\text { Gum } \\
\text { Kondagogu } \\
\text { extract }\end{array}$ & 15 & - & Klebsiella Pneumoniae & Suresh et al. (2016) \\
\hline CuO NPs & Thymbra spicata & Leaf extract & 26.8 & $100-200 \mu \mathrm{g} / \mathrm{ml}$ & S.aureus & Erci et al. (2020) \\
\hline CuO NPs & Cymbopogon citratus & Leaf extract & $14.5 \pm 2.0$ & $2000 \mu \mathrm{g} / \mathrm{ml}$ & E. coli-336 and MRSA-1 & $\begin{array}{l}\text { Cherian et al. } \\
(2020)\end{array}$ \\
\hline $\mathrm{Cu} N \mathrm{NP}$ & $\begin{array}{l}\text { Cardiospermum } \\
\text { halicacabum }\end{array}$ & Leaf extract & $30-40$ & $100 \mu \mathrm{g} / \mathrm{ml}$ & P. aeruginosa, S. aureus, and E. coli & $\begin{array}{l}\text { Punniyakotti et al. } \\
\text { (2020) }\end{array}$ \\
\hline CuO NPs & Eucalyptus globulus & Leaf extract & $16.78-22.5$ & $125-2000 \mu \mathrm{g} / \mathrm{ml}$ & $\begin{array}{l}\text { E. coli-336, P. aeruginosa-621 and } \\
\text { MRSA-1 }\end{array}$ & Ali et al. (2019) \\
\hline $\mathrm{TiO}_{2}$ & Withania somnifera & Root extract & 247 & - & $\begin{array}{l}\text { P.aeruginosa, E.coli, Listeria } \\
\text { monocytogenes, MRSA, serratia } \\
\text { marcescens, and Candida albicans }\end{array}$ & $\begin{array}{l}\text { Al-Shabib et al. } \\
\text { (2020) }\end{array}$ \\
\hline $\mathrm{TiO}_{2}$ & Ocimum sanctum & Leaf extract & $90-100$ & $250-650 \mu \mathrm{g} / \mathrm{ml}$ & $\begin{array}{l}\text { P. aeruginosa, E. coli, S. aureus and } C \text {. } \\
\text { albicans }\end{array}$ & $\begin{array}{l}\text { Dan and Khan } \\
\text { (2019) }\end{array}$ \\
\hline $\mathrm{TiO}_{2}$ & Rosa davurica & Leaf extract & $146 \pm 3$ & $15.62 \mu \mathrm{g} / \mathrm{ml}$ & $\begin{array}{l}\text { S. aureus, B. cereus, S. enterica, and } \\
\text { E. coli }\end{array}$ & Jin et al. (2021) \\
\hline $\mathrm{TiO}_{2}$ & Ochradenus arabicus & Leaf extract & - & - & $P$. aeruginosa & Zubair et al. (2021) \\
\hline $\mathrm{TiO}_{2}$ & Aloe barbadensis & Leaf extract & 20 & $31.25 \mu \mathrm{g} / \mathrm{ml}$ & P. aeruginosa & $\begin{array}{l}\text { Rajkumari et al. } \\
\text { (2019) }\end{array}$ \\
\hline $\mathrm{TiO}_{2}$ & $\begin{array}{l}\text { Ficus benghalensis, } \\
\text { Azadirachta indica twigs, } \\
\text { and Syzygium aromaticum }\end{array}$ & $\begin{array}{l}\text { Twigs and bud } \\
\text { extract }\end{array}$ & $10-33$ & $100 \mu \mathrm{g} / \mathrm{ml}$ & $\begin{array}{l}\text { Citrobacter freundii, Streptococcus } \\
\text { mutans and, Candida albicans }\end{array}$ & $\begin{array}{l}\text { Achudhan et al. } \\
\text { (2020) }\end{array}$ \\
\hline $\mathrm{Fe}_{2} \mathrm{O}_{3} \mathrm{NPs}$ & $\begin{array}{l}\text { polyherbal ayurvedic drug } \\
\text { formulation, Liv } 52\end{array}$ & Plant extract & - & - & $\begin{array}{l}\text { MRSA, MDR P. aeruginosa and } C \text {. } \\
\text { albicans }\end{array}$ & $\begin{array}{l}\text { Ansari and Asiri, } \\
(2021)\end{array}$ \\
\hline
\end{tabular}


TABLE 1 | (Continued) List of phytosynthesized nanoparticles exhibiting antibiofilm activity

\begin{tabular}{|c|c|c|c|c|c|c|}
\hline Nanoparticles & Plants source & Plant tissue & Size (nm) & $\begin{array}{l}\text { Concentration for } \\
\text { anti-biofilm } \\
\text { activity }\end{array}$ & $\begin{array}{l}\text { Biofilm producing } \\
\text { microorganisms }\end{array}$ & References \\
\hline FeO NPs & Avicennia marina & Leaf extract & $10-25$ & $200 \mu \mathrm{g} / \mathrm{ml}$ & $\begin{array}{l}\text { Escherichia coli, Staphylococcus } \\
\text { aureus, and Pseudomonas aeruginosa }\end{array}$ & $\begin{array}{l}\text { Ramalingam et al. } \\
\text { (2019) }\end{array}$ \\
\hline FeO NPs & Thymbra spicata & Leaf extract & 93.9 & $100 \mu \mathrm{g} / \mathrm{ml}$ & S. aureus & $\begin{array}{l}\text { Erci and Cakir-Koc, } \\
(2020)\end{array}$ \\
\hline $\mathrm{CeO}_{2}-\mathrm{NPS}$ & Acorus calamus & Plant extract & 22.03 & - & Biofilm producing bacteria & Altaf et al. (2021) \\
\hline $\mathrm{Sn}-\mathrm{CeO}_{2} \mathrm{NPs}$ & Pometia pinnata & Leaf extract & $15-20$ & $512 \mu \mathrm{g} / \mathrm{ml}$ & S. aureus and Listeria monocytogenes & Naidi et al. (2021b) \\
\hline $\begin{array}{l}\mathrm{Zr} / \mathrm{Sn}- \\
\mathrm{CeO}_{2} \mathrm{NPs}\end{array}$ & Pometia pinnata & Leaf extract & $12-17$ & $512 \mu \mathrm{g} / \mathrm{ml}$ & S. aureus and Listeria monocytogenes & Naidi et al. (2021a) \\
\hline $\mathrm{Ti}-\mathrm{CeO}_{2}$ & Phoenix dactylifera & Fruit extract & $7-9$ & $1000 \mu \mathrm{g} / \mathrm{ml}$ & P. aeruginosa & Ahmed et al. (2020) \\
\hline Pd NPs & Orthosiphon stamineus & Leaf extract & 110 & $\begin{array}{l}15.25 \pm \\
0.012 \mu \mathrm{g} / \mathrm{ml}\end{array}$ & $\begin{array}{l}\text { Methicillin sensitive staphylococcus } \\
\text { aureus (MSSA) strain }\end{array}$ & (N et al., 2021) \\
\hline Se NPs & Psidium guajava & Leaf extract & $30-50$ & $25 \mathrm{mg} / \mathrm{ml}$ & Enterococcus faecalis & $\begin{array}{l}\text { Miglani and } \\
\text { Tani-Ishii (2021) }\end{array}$ \\
\hline $\mathrm{NiO}$ NPs & Eucalyptus globulus & Leaf extract & $10-20$ & $0.1-1.6 \mathrm{mg} / \mathrm{ml}$ & $\begin{array}{l}\text { ES } \beta L(+) E \text {. coli and } P \text {. aeruginosa and } \\
\text { methicillin-sensitive and resistant } S \text {. } \\
\text { aureus }\end{array}$ & Saleem et al. (2017) \\
\hline
\end{tabular}

TABLE 2 | List of microbially synthesized nanoparticles exhibiting antibiofilm activity.

\begin{tabular}{|c|c|c|c|c|c|}
\hline Nanoparticles & Microbial source & Size (nm) & $\begin{array}{l}\text { Concentration for anti- } \\
\text { biofilm activity }\end{array}$ & Biofilm producing microorganisms & References \\
\hline Ag NPs & Lactobacillus fermentum & 6 & $7.8 \mathrm{mg} / \mathrm{L}$ & $P$. aeruginosa & Zhang et al. (2014) \\
\hline Ag NPs & Escherichia coli & 33.6 & $10 \mathrm{mM}$ & E. coli, S. aureus, P. aeruginosa, K. pneumoniae & $\begin{array}{l}\text { Neihaya and Zaman } \\
(2018)\end{array}$ \\
\hline Ag NPs & Klebsiella oxytoca & - & - & S. aureus, $P$. aeruginosa & $\begin{array}{l}\text { Cusimano et al. } \\
(2020)\end{array}$ \\
\hline Ag NPs & Weissella oryzae & $10-30$ & $5-6 \mu \mathrm{g} / \mathrm{ml}$ & S. aureus and $P$. aeruginosa & Singh et al. (2016a) \\
\hline Ag NPs & Sporosarcina forensis & 102 & $3-6.9 \mu \mathrm{g} / \mathrm{ml}$ & S. aureus, $P$. aeruginosa, E. coli & Singh et al. (2016d) \\
\hline Ag NPs & $\begin{array}{l}\text { Pseudomonas } \\
\text { deceptionensis }\end{array}$ & $10-30$ & $5 \mu \mathrm{g} / \mathrm{L}$ & S. aureus and $P$. aeruginosa & Jo et al. (2016) \\
\hline Ag NPs & Streptomyces sp. & 11 & $3.9-31.25 \mathrm{lg} / \mathrm{ml}$ & P. aeruginosa & $\begin{array}{l}\text { Bakhtiari-Sardari et al. } \\
(2020)\end{array}$ \\
\hline Ag NPs & Pseudomonas sp. & $10-40$ & $10 \mu \mathrm{g} / \mathrm{ml}$ & S. aureus, $P$. aeruginosa & Singh et al. (2018a) \\
\hline $\mathrm{ZnO} \mathrm{NPs}$ & Pseudomonas putida & $25-45$ & $10 \mu \mathrm{g} / \mathrm{ml}$ & $\begin{array}{l}\text { Pseudomonas otitidis, Pseudomonas oleovorans, } \\
\text { Acinetobacter baumannii, Bacillus cereus, and } \\
\text { Enterococcus faecalis }\end{array}$ & $\begin{array}{l}\text { Jayabalan et al. } \\
\text { (2019) }\end{array}$ \\
\hline ZnO NPs & E. faecalis & $16-96$ & $2-128 \mu \mathrm{g} / \mathrm{ml}$ & E. coli, K. pneumoniae, P. aeruginosa & $\begin{array}{l}\text { Ashajyothi et al. } \\
\text { (2016) }\end{array}$ \\
\hline Selenium NPs & Bacillus sp & $80-220$ & $0-100 \mu \mathrm{g} / \mathrm{ml}$ & $\begin{array}{l}\text { Staphylococcus aureus, Pseudomonas aeruginosa, and } \\
\text { Proteus mirabilis }\end{array}$ & Shakibaie et al. (2015) \\
\hline TiO2 NPs & Bacillus sp. & $10-30$ & - & Environmental aquatic strains & $\begin{array}{l}\text { Dhandapani et al. } \\
\text { (2012) }\end{array}$ \\
\hline Cu NPs & E. faecalis & $12-90$ & $2-128 \mu \mathrm{g} / \mathrm{ml}$ & $\begin{array}{l}\text { E. coli, K. pneumoniae, } P \text {. aeruginosa, S. flexneri, S. } \\
\text { aureus, E. faecalis }\end{array}$ & $\begin{array}{l}\text { Ashajyothi et al. } \\
\text { (2016) }\end{array}$ \\
\hline Au NPs & $\begin{array}{l}\text { Caldicellulosiruptor } \\
\text { changbaiensis }\end{array}$ & $\begin{array}{l}1.03,2.43 \\
\text { and } 20\end{array}$ & - & S. aureus, $P$. aeruginosa & Bing et al. (2018) \\
\hline Au NPs & Acinetobacter baumannii & 15 & $0.002 \mathrm{~mol} / \mathrm{L}$ & P. aeruginosa, V. cholera, Brevibacterium linens & $\begin{array}{l}\text { Rajput and Bankar } \\
(2017)\end{array}$ \\
\hline
\end{tabular}

against Chromobacterium violaceum and further studied the potential effect of $\mathrm{Pb}$-AgNPs on QS-regulated phenotypes in PAO1 Pseudomonas aeruginosa. The data revealed that the phytofabricated $\mathrm{Pb}$-AgNPs remarkably inhibited the QS-mediated virulence factors such as violacein of $C$. violaceum, elastase, and pyocyanin of $P$. aeruginosa. Moreover, these nanostructures imparted significant antibiofilm activity against $P$. aeruginosa, thereby suggesting their potential role in overcoming bacterial resistance against conventional antibiotics (Shah et al., 2019). In a similar study done by Srinivasan et al., the anti-QS and antibiofilm potential of Piper betle leaf extract mediated silver nanoparticles (PbAgNPs) against S. marcescens 
and P. mirabilis was evaluated. The data revealed PbAgNPs mediated inhibition of QS-related virulence factors such as protease, prodigiosin, exopolysaccharides, biofilm formation, and hydrophobicity productions in uropathogens. Further, the genomic analysis reported downregulated expression of $\mathrm{flhD}$, $\mathrm{flhB}$, and rsbA genes in $P$. mirabilis and fimC, fimA, flhD, and bsmB genes in S. marcescens respectively (Srinivasan et al., 2018).

Ali et al. have developed biogenic silver nanoparticles (AgNPs) employing aqueous leaf extract of Eucalyptus globulus (ELE) and evaluated their antibiofilm and antibacterial potential. After $24 \mathrm{~h}$ of treatment, biofilms produced by $S$. aureus and $P$. aeruginosa were shown to be inhibited by $82 \pm 3 \%$ and $81 \pm 5 \%$, respectively, at $30 \mu \mathrm{g} / \mathrm{ml}$ (Ali et al., 2015). In 2018, research was done by Muthamil et al., phyto-synthesized silver nanoparticles (AgNPs), prepared using methanolic leaf extracts of Dodonaea viscosa and Hyptis suoveolens were assessed for their antibiofilm properties against Candida spp. AgNPs obtained from both the extract showed significant biofilm inhibitory properties with about $88 \%$ of biofilm reduction at a concentration of $10 \mu \mathrm{g} / \mathrm{ml}$, as evidenced by microscopic analysis and in vitro virulence assays (Muthamil et al., 2018).

Sarathi Kannan et al. demonstrated the potential biofilm inhibitory activity of Ludwigia octovalvis leaf extracts derived silver nanoparticles (AgNPs) against Staphylococcus epidermidis and Pseudomonas aeruginosa. It was noticed that at $100 \mu \mathrm{g} / \mathrm{ml}$, AgNPs imparted the highest antibiofilm activity of 62.42 and $50.62 \%$ against $S$. epidermidis and $P$. aeruginosa respectively (Sarathi Kannan et al., 2021). Moreover, Ali et al. biofabricated silver nanoparticles using bark extract of medicinal plant Holarrhena pubescens (HP-AgNPs) and screened their antibacterial and antibiofilm activity against imipenem-resistant Pseudomonas aeruginosa clinical isolates. The HP-AgNPs exhibited antibacterial and antibiofilm activity in a dose-dependent fashion as confirmed by the confocal laser scanning microscopy (Ali et al., 2018).

Also, Vijayakumar et al. prepared silver nanoparticles using garlic clove extract (G-AgNPs) and evaluated its broad-spectrum therapeutic activity including antibiofilm properties. They demonstrated that G-AgNPs $(100 \mu \mathrm{g} / \mathrm{ml})$ imparted significant antibacterial as well as anti-biofilm activity against clinically relevant pathogens like methicillin-resistant $S$. aureus and Pseudomonas aeruginosa (Vijayakumar et al., 2019). Arya et al. fabricated bio-inspired AgNPs utilizing aqueous leaf extract of Prosopis juliflora and evaluated its biofilm inhibitory properties. The data obtained from congo red agar (CRA) plate assay showed the synthesized AgNPs exhibited significant antibiofilm activity against Bacillus subtilis and Pseudomonas aeruginosa (Arya et al., 2019).

Moreover, Bharathi and her coworkers reported that silver nanoparticles synthesized using fruit extract of Cordia dichotoma (Cd-AgNPs) lead to a significant reduction in S.aureus and E.coli biofilms by 92 percent and 95 percent, respectively, at $100 \mu \mathrm{g} / \mathrm{ml}$ concentration (Bharathi et al., 2018). Also, Majumdar et al. developed silver nanoparticles (CM-AgNPs) using fruit extract of Citrus macroptera (CM) and explored their antibiofilm potential. The biosynthesized CMAgNPs efficiently inhibited the development of biofilm in a dose-dependent manner with
70 and 80 percent of biofilm inhibition in Bacillus subtilis and Pseudomonas aeruginosa respectively, at the highest concentration (260 nM) (Majumdar et al., 2020). Interestingly, Eric and Torlak synthesized Ag nanoparticles (AgNPs) by using the aqueous leaf extract of Thymus serpyllum. The antibiofilm data revealed that AgNPs $(100 \mu \mathrm{g} / \mathrm{ml})$ inhibited the biofilm formation by $73 \%$ in S. aureus (Erci and Torlak, 2019).

Juan et al. synthesized silver nanoparticles by using extract of benzoin gum as bioreductant and capping agent and studied their antibiofilm activity against biofilm-producing E. coli strain. At 1, 2,5 , and $10 \mu \mathrm{g} / \mathrm{ml}$ concentrations, treatment for $24 \mathrm{~h}$ resulted in a reduction in biofilm development of about 10.9, 44.1, 54.0, and 65.5 percent, respectively thereby indicating that the AgNPs were capable to inhibit the biofilm development in E. coli (Du et al., 2016). Further, Prasad and his coworkers reported phytogenic synthesis of silver nano bactericides by utilizing Acorus calamus $L$. extracts. The biofilm inhibitory activity was evaluated against Helicobacter pylori clinical isolates. The synthesized NPs exhibited significant antibiofilm activity at concentration $350 \mu \mathrm{g} / \mathrm{ml}$, as evidenced by crystal violet and ruthenium red assays (Prasad et al., 2019).

Interestingly, Gupta and his coworkers showed silver nanoparticles synthesized using methanolic leaf extract of Syzygium cumini have the potential to impede biofilm development of $P$. aeruginosa, S. aureus, and C. albicans in a concentration-dependent manner. At $250 \mu \mathrm{g} / \mathrm{ml}$, AgNPs inhibited more than $90 \%$ biofilm formation, while at $125 \mu \mathrm{g} /$ $\mathrm{ml}$ concentration, $85 \%$ biofilm inhibition was recorded (Gupta et al., 2014a). Moreover, Al-Ansari et al. reported the antibiofilm and antibacterial activity of Lilium lancifolium leaf extract mediated silver nanoparticles against Streptococcus pneumonia and Pseudomonas aeruginosa. Treatment with $25 \mu \mathrm{l}$ of AgNPs imparted antibacterial activity while treatment with $50 \mu$ l showed significant antibiofilm properties, as evident from crystal violet assay and confocal micrographic images (Al-Ansari et al., 2019).

Interestingly, Barbadi et al. compared the antibiofilm activity of Zataria multiflora aerial extract-mediated silver nanoparticles (P-AgNPs) and commercial silver nanoparticles (C-AgNPs) against Staphylococcus aureus ATCC 25923 bacteria. The data showed dose-dependent biofilm inhibitory activity, with excellent inhibition at concentration $\geq 8 \mu \mathrm{g} / \mathrm{ml}$ for both P-AgNPs and C-AgNPs. Further, they showed that plant-derived AgNPs (P-AgNPs) imparted higher biofilm inhibitory properties at lower concentrations as compared to C-AgNPs (Barabadi et al., 2021). Similarly, Moulavi et al. stated that phytofabricated AgNPs using Artemisia scoparia as bioreductant imparted relatively more antibiofilm activity as compared to commercial AgNPs against methylationdependent restriction system (MDRS) strains. The mean MIC value for the commercial and biosynthetic AgNPs was found to be 12.81 and $8.45 \mu \mathrm{g} / \mathrm{ml}$ respectively, on MDRS isolates. Also, the biofabricated AgNPs exhibited superior antibiofilm activity against MDRS via unknown mechanisms (Moulavi et al., 2019).

Further, Gupta et al. showed that silver nanoparticles (AgNPs) obtained via green synthesis using leaf biomass of Psidium guajava have a remarkable ability to inhibit biofilm development of S. aureus, E. coli, and C. albicans by 96, 90, 
and $75 \%$ respectively, as evident from crystal violet assay (Gupta et al., 2014b). Recently, Almatroudi et al. assessed the antibiofilm activity of silver nanoparticles (Ns-AgNPs) biosynthesized using seed extract of Nigella sativa. They showed that at concentration $12.5 \mu \mathrm{g} / \mathrm{ml}$, Ns-AgNPs limited the biofilm development by $84.92 \%$ for E. coli, $88.42 \%$ for Enterococcus faecalis, $81.86 \%$ for Klebsiella pneumonia, $82.84 \%$ for Staphylococcus aureus, and 49.9\% for Pseudomonas aeruginosa, respectively (Almatroudi et al., 2020).

Interestingly, Kulshrestha et al. developed a bioinspired graphene oxide-silver nanocomposite (GO-Ag) by employing an eco-friendly route using Lagerstroemia speciosa (L.) Pers floral extract. It was observed that at concentrations 24 and $12 \mu \mathrm{g} / \mathrm{ml}$, GO-Ag resulted in 90 and $49 \%$ biofilm reduction respectively, in Enterobacter cloacae. Similarly, 89 and 34\% biofilm declination was observed in Streptococcus mutans when exposed to 47 and $24 \mu \mathrm{g} / \mathrm{ml}$ of $\mathrm{GO}-\mathrm{Ag}$ respectively (Kulshrestha et al., 2017). Accordingly, Korkmaz et al. have synthesized silver nanoparticles (AgNPs) with the help of Mimusops elengi liquid fruit extract. According to their results, AgNPs $(1250 \mu \mathrm{g} / \mathrm{ml})$ inhibited $86.36 \%$ of the biofilm formation in Escherichia coli (Korkmaz et al., 2020).

In a recent study, Nersin et al. evaluated the antibiofilm activity of biosynthesized AgNPs prepared using aqueous leaf extracts of Rhododendron ponticum. They found that almost at each concentration AgNPs imparted biofilm inhibitory activity in a dose-dependent manner. The highest concentration of AgNPs which showed maximum antibiofilm activity was $1.0 \mathrm{mg} / \mathrm{ml}$ (Nesrin et al., 2020).

Accordingly, biogenic silver nanoparticles (Ag@CC-NPs) were fabricated using aqueous extract of Carum copticum seed by Qais et al. The synthesized Ag@CC-NPs imparted biofilm inhibitory activity with $86.3 \%, 77.6 \%$, and $75.1 \%$ biofilm inhibition of $S$. marcescens, $P$. aeruginosa, and $C$. violaceum respectively. Further at sub-MIC, Ag@CC-NPs inhibited the production of virulence factors such as pyoverdin, pyocyanin, swimming motility, elastase activity, exoprotease activity, and rhamnolipid in $P$. aeruginosa by $49.0,76.9,89.5,53.3,71.1$, and $60.0 \%$ respectively. Moreover, virulence factors of $S$. marcescens viz. swarming motility, exoprotease activity, and prodigiosin production was reduced by $90.7,67.8$, and $78.4 \%$ respectively. Further, the SEM and CLSM observations showed a remarkable reduction in biofilm development on glass coverslip (Qais et al., 2020). Further, Foroohimanjili et al. investigated the antibiofilm, antibacterial, and anti quorum sensing properties of phytosynthesized silver nanoparticles (AgNPs) prepared using leaf extract of Mespilus germanica against multidrug-resistant Klebsiella pneumoniae strains. Their work revealed that at subMIC, AgNPs prominently inhibited the establishment of biofilm in all the biofilm-producing strains (Foroohimanjili et al., 2020).

In another study silver nanoparticles, synthesized employing aqueous leaf extracts of Moringa oleifera Lam imparted excellent biofilm eradication potential (78\%) for P. aeruginosa whereas only $43 \%$ for S. epidermidis, as reported by Gokul Brindha (Gokul Brindha et al., 2020).

Interestingly, Muthuraman et al. employed a green route for the synthesis of silver nanoparticles (AgNPs) using medicinally relevant Nardostachys jatamansi rhizome extract. They showed that AgNPs $(64 \mu \mathrm{M})$ prepared using heated plant extract exhibited superior antibiofilm activity against $P$. aeruginosa and $S$. aureus. Whereas AgNPs prepared using stirred plant extract required a relatively higher dose $(500 \mu \mathrm{M})$ to impart their anti-biofilm effect (Muthuraman et al., 2019). In another study, Mohanta and colleagues developed biocompatible silver nanoparticles (AgNPs) utilizing foliage extracts of three different plants namely Glochidion lanceolarium, Semecarpus anacardium, and Bridelia retusa. The phytosynthesized AgNPs were screened for antibacterial and anti-biofilm activity against Staphylococcus aureus, Pseudomonas aeruginosa, and Escherichia coli; their data indicated promising results (Mohanta et al., 2020).

Further, Govindappa et al. assessed the antibiofilm activity of silver nanoparticles synthesized using fleshy pericarp of Punica granatum (Pfp-AgNPs). The Pfp-AgNPs were investigated for the antibiofilm effectiveness against Pseudomonas aeruginosa. The outcome revealed that the nanoformulations significantly increased the toxicity level in $P$. aeruginosa in a concentrationdependent fashion and led to potassium leakage, cellular damage, and biofilm inhibitory activity (Govindappa et al., 2021).

Interestingly, $\mathrm{Al}$ Aboody et al. synthesized silver nanoparticles (AgNP) using latex of Azadirachta indica. They demonstrated the antibiofilm activity of AgNPs against Fluconazole resistant clinical isolate of Candida tropicalis and concluded that at concentrations of 4.12 and $3.25 \mu \mathrm{g} / \mathrm{ml}$, AgNPs inhibited the biofilms of fluconazole-resistant and fluconazole-susceptible $C$. tropicalis, respectively (Al Aboody, 2019). Velgosova et al. evaluated AgNPs and nanocomposites doped with AgNPs against biofilm development. For this, they synthesized AgNPs via green route using leaf extract of Rosmarinus officinalis. And further, doped Polyvinyl alcohol (PVA) matrix with green synthesized AgNPs to obtain polymer matrix composite (PMC) microfibers. The antibiofilm efficacy was screened against biofilm-producing one-cell green algae Parachlorella kessleri. The results revealed that there was no antibiofilm effect of pure PVA matrix whereas AgNPs and PMC (PVAAgNP composites) microfibers showed significant antibiofilm activity (Velgosova et al., 2021).

\section{Gold Nanoparticles}

Gold nanoparticles (AuNPs) are one of the most extensively exploited metal nanoparticles (NPs) paving their way in various fields of science and industry. Due to its various therapeutic properties like antibacterial, anticancer, antimalarial, and antibiofilm, there is a surge in their demand. To fulfill such demands, rigorous research is required to optimize its synthesizing approaches. Among various methods employed for the synthesis of AuNPs, a biogenic approach using plant extract is simple, effective, cheap, and eco-friendly (Ali et al., 2020). In this regard, Ali et al. developed gold nanoparticles utilizing Tinospora cordifolia plant stem (Ayurvedic medicinal plant) and assessed its biofilm inhibitory properties against Pseudomonas aeruginosa PAO1 biofilm. The SEM and crystal violet assay data revealed that the sub-MIC value of AuNPs significantly reduced the biofilm-producing capability of $P$. aeruginosa in a concentration-dependent manner. Their data 
showed that biofilm formation was inhibited up to 59.9, 36.6, $27.1 \%$ at 150,100 , and $50 \mu \mathrm{g} / \mathrm{ml}$ concentrations of AuNPs respectively. The CLSM analysis further indicated that the structure of the biofilm in the sub-MIC of AuNPs had abnormalities. Also, treatment of PAO1 with AuNPs at $150 \mu \mathrm{g} / \mathrm{ml}$ displayed internalization of the nanoparticles (Ali et al., 2020). Similarly, Manju et al., showed that gold nanoparticles biosynthesized applying essential oil of Nigella sativa (NsEO-AuNPs), efficiently inhibited the biofilm establishment of Staphylococcus aureus and Vibrio harveyi by reducing the hydrophobicity index (78 and $46 \%$ respectively) (Manju et al., 2016).

In another study, Vijayakumar et al. developed AuNPs using Musa paradisiaca peel extract (MPPE-AuNPs) and studied its biofilm inhibitory properties in multiple antibiotic-resistant Enterococcus faecalis. Confocal light microscopic observations demonstrated that the MPPE-AuNPs meritoriously repressed the biofilm formed by E. faecalis at concentration $100 \mu \mathrm{g} / \mathrm{ml}$ (Vijayakumar et al., 2017). Interestingly, Qais et al. biosynthesized gold nanoparticles by employing an aqueous fruit extract of Capsicum annuum (AuNPs-CA) and assessed its efficacy against the QS-regulated virulence factors and biofilms of Serratia marcescens and Pseudomonas aeruginosa. The result showed a significant reduction of QS-mediated virulent traits of $P$. aeruginosa PAO1 such as pyoverdin, elastase activity, exoprotease activity, pyocyanin, swimming motility, and rhamnolipids production by $72.16,65.72,81.12,91.94,46.09$, $46.66 \%$, respectively. Further, microscopic studies showed that the growth and synthesis of exopolysaccharides have been suppressed due to reduced bacterial adhesion and colonization on a solid substrate (Qais et al., 2021).

Accordingly, Perveen and colleagues fabricated biogenic gold nanoparticles by utilizing the seed extract of Trachyspermum ammi (TA-AuNPs), followed by assessing its effectiveness against biofilms of Serratia marcescens and Listeria monocytogenes. It was demonstrated that there was substantial anti-biofilm activity against both $S$. marcescens (81\%) and L. monocytogenes (73\%). Moreover, the key factors of biofilm development and maintenance such as cell surface hydrophobicity, exopolysaccharide, and motility were considerably inhibited at sub-MICs. Also, the NPs efficiently eradicated preformed established biofilms of L. monocytogenes and S. marcescens by 58 and 64\%, respectively (Perveen et al., 2021).

\section{Zinc Oxide Nanoparticles}

Among several metallic nanoparticles, zinc oxide nanoparticles have held the attention of several researchers owing to their multifunctional properties and versatility. The antibacterial efficacy of zinc oxide nanoparticles has been well established against both gram-negative and gram-positive bacteria as well as against fungi. It has already been reported that $\mathrm{ZnO}$ NPs are nontoxic to human cells while imparting antibacterial properties via several mechanisms like the generation of ROS, the release of metal ions, etc (Gudkov et al., 2021). Further, green synthesized zinc oxide nanoparticles have been known to impart superior properties due to their eco-friendly characteristics. Regarding this several plant-based syntheses of $\mathrm{ZnO}$ NPs have been carried and their antibacterial and antibiofilm efficacies have been evaluated.

Interestingly, Vijayakumar et al. biologically developed Plectranthus amboinicus mediated zinc oxide nanoparticles (Pam-ZnO NPs). Their data showed that the synthesized nanoparticles exhibited antibiofilm activity at concentrations range $8-10 \mu \mathrm{g} / \mathrm{ml}$ against the biofilms of methicillin-resistant Staphylococcus aureus. Further, Confocal laser scanning microscopy analysis suggested that the biofilm-forming ability of $S$. aureus is intensely inhibited by Pam-ZnO NPs (Vijayakumar et al., 2015).

Similarly, in their other study, they reported the QS inhibitory property of zinc oxide nanoparticles synthesized using seed extract of Nigella sativa (NS-ZnNPs). Their fabricated nanoparticles inhibited QS-mediated functions of $C$. violaceum and inhibited the production of pyocyanin, alginate, protease, and elastase in $P$. aeruginosa PAO1 evidently. Also, at sub-MICs, NSZnNPs prevented the biofilm development as well as eradicated pre-formed biofilms of food-borne bacteria viz. C. violaceum 12472, PAO1, L. monocytogenes, E. coli. Preformed biofilms were eradicated by $66,78,68$, and $72 \%$ in E. coli, $P$. aeruginosa, $C$. violaceum, and, L. monocytogenes respectively as observed by $\mathrm{CV}$ assay. Further, CLSM also confirmed the antibiofilm property of NS-ZnNPs (Al-Shabib et al., 2016).

Further, Al-Shabib et al. investigated the protein-binding and antibiofilm activity of zinc oxide nanoparticles synthesized from Ochradenus baccatus leaf extract (OB-ZnNPs). The synthesized OB-ZnNPs imparted considerable antibiofilm properties against human and foodborne pathogens (Listeria monocytogenes Chromobacterium violaceum, Serratia marcescens, $P$. aeruginosa, Klebsiella pneumoniae, and Escherichia coli) at sub-MICs. Further, the NPs significantly impaired swarming motility and EPS production which aids in biofilm development. Their data showed the highest inhibition of biofilm in $P$. aeruginosa by $84 \%$; in $E$. coli by $67 \%$; in $L$. monocytogenes by $78 \%$; in $K$. pneumonia by $70 \%$; in $S$. marcescens by $80 \%$; and, in C. violaceum by $64 \%$ (Al-Shabib et al., 2018).

Accordingly, Dogan and Kocabas synthesized zinc oxide ( $\mathrm{ZnO})$ nanoparticles (NPs) using foliage extracts of Veronica multifida under various physical conditions. The antibiofilm activity of the prepared NPs was evaluated against $S$. aureus and $P$. aeruginosa. The results revealed that in $S$. aureus, $10 \mu \mathrm{g} / \mathrm{ml}$ of $\mathrm{ZnO}$ NPs at $\mathrm{pH} 7$ inhibited $88 \%$ of biofilm development, and $50 \mu \mathrm{g} / \mathrm{ml} \mathrm{ZnO} \mathrm{NPs}$ at $\mathrm{pH} 12$ inhibited $87 \%$ of the biofilm development. While in $P$. aeruginosa, at both $\mathrm{pH} 7$ and $\mathrm{pH}$ 12 , the maximum biofilm inhibitory activity was observed at a concentration of $50 \mu \mathrm{g} / \mathrm{ml}$ (Doğan and Kocabaş, 2020). Further, Alavi et al. demonstrated the antibiofilm efficacy of zinc oxide NPs synthesized using Artemisia haussknechtii leaves extract. Their crystal violet (CV) assay analysis revealed a significant reduction in biofilm formation for S. epidermidis with $63.43 \%$ $(0.562 \pm 0.015)$ and $P$. aeruginosa with $62.88 \%(0.582 \pm 0.025)$ at $100 \mu \mathrm{g} / \mathrm{ml} \mathrm{ZnO} \mathrm{NPs.} \mathrm{Further,} \mathrm{Light} \mathrm{microscopic} \mathrm{observations}$ showed there was a significant reduction of biofilm formation with the increase in ZnO NPs concentration (Alavi et al., 2019). 
Interestingly, Vinotha et al. reported a novel fabrication of $\mathrm{ZnO}$ nanoparticles by utilizing foliage extract of Costus igneus (Ci-ZnO NPs). Their prepared $\mathrm{Ci}-\mathrm{ZnO}$ NPs displayed encouraging antibiofilm and antibacterial activities against $L$. fusiformis, S. mutans, V. parahaemolyticus, and P. vulgaris bacteria at concentrations 75 and $100 \mu \mathrm{g} / \mathrm{ml}$. Further, the light microscopy and CLSM analysis revealed disintegration and reduced growth of biofilm in a concentration-dependent fashion. Also, they suggested that the biofilm inhibitory activity of $\mathrm{ZnO}$ NPs was due to the generation of ROS and the action of surface ions released from the nanoparticles (Vinotha et al., 2019).

Similarly, Malaikozhundan et al. synthesized Withania somnifera leaf extract-assisted $\mathrm{ZnO} \mathrm{NPs}$ (Ws-ZnO NPs) and evaluated its antibiofilm efficacy against $S$. aureus and $E$. faecalis. They showed that at concentration $100 \mu \mathrm{g} / \mathrm{ml}$, the WsZnO NPs imparted significant biofilm inhibitory activity. Further, Ws-ZnO NP's surface activity resulted in disruption of the bacterial cell wall and biofilm inhibition. Also, excessive generation of ROS leads to enhanced antibacterial properties (Malaikozhundan et al., 2020). Accordingly, Cherian et al. developed bio-inspired zinc oxide nanoparticles (MFLE-ZnONPs) fabricated by using Myristica fragrans leaf ester (MFLE) and assessed its antibiofilm activity against methicillin-resistant Staphylococcus aureus and multi-drug resistant Escherichia coli clinical isolates. It was found that the synthesized nanoparticles showed dosedependent declination of biofilm development in both the tested bacterial strain (Cherian et al., 2019).

Employing the alcoholic extract of Artemisia, Galederi and Teimouri fabricated $\mathrm{ZnO}-\mathrm{NPs}$ and studied its inhibitory effects on biofilm development by $P$. aeruginosa strains. The outcomes suggested that $\mathrm{ZnO}-\mathrm{NPs}$ were effective on the isolates at the minimum and maximum viscosities of 3.125 and $100 \mathrm{mg} / \mathrm{ml}$, respectively. (Galedari and Teimouri, 2020).

Moreover, Obeizi et al. reported the antibiofilm efficacy of biogenic zinc oxide nanoparticles prepared by using Eucalyptus globulus essential oil. Their results revealed that $\mathrm{ZnO}$ NPs effectively inhibited biofilm development in S. aureus ATCC 25923 and $P$. aeruginosa ATCC 27853 in a dose-dependent manner. The percentage of biofilm inhibition at $100 \mu \mathrm{g} / \mathrm{ml}$ $\mathrm{ZnO}$ NPs was observed to be 97 and $85 \%$ against $P$. aeruginosa biofilm and S. aureus, respectively (Obeizi et al., 2020).

Accordingly, Basumatari et al. fabricated zinc oxide nanoparticles (ZnONPs) by utilizing an aqueous pseudostem extract of Musa balbisiana Colla ash (AEPA), a plant biowaste, and evaluated its efficacy as an antibiofilm and antibacterial agent. The AEPA mediated $\mathrm{ZnO}$ NPs exhibited note-worthy antibiofilm activity against $P$. aeruginosa, as revealed by 96-microtitre well plate and Congo red agar method. At a dosage of $100 \mu \mathrm{g} / \mathrm{ml}$, the percent inhibition for $P$. aeruginosa was $95.13 \%$, indicating an enhanced inhibitory impact on bacterial biofilm breakdown (Basumatari et al., 2021).

\section{Copper and Copper Oxide Nanoparticles}

The U.S. Environmental Protection Agency (USEPA) has designated elemental copper and its compounds as antibacterial materials. Nanoformulations of Copper oxide exhibit enhanced antimicrobial activity towards pathogenic microorganisms. Further, numerous reports have also discussed the antibiofilm activity of copper oxide nanoparticles (Mahmoodi et al., 2018). Green synthesized $\mathrm{CuO}$ nanoparticles have been known to impart superior properties due to their less toxic nature.

Regarding this, Naseer et al. demonstrated the antibiofilm efficacy of biogenic copper oxide nanoparticles (CuO NPs) prepared by utilizing Melia azedarach and Cassia fistula leaf extracts. The results revealed that at concentration $1 \mu \mathrm{g} / \mathrm{ml}$ the $M$. azadarech derived NPs the prevented biofilm development of H. pylori and K. pneumoniae by 99.5 and $92.5 \%$ respectively. While, at the same concentration, the C. fistula-derived NPs inhibited biofilm inhibition by 100 and $99.8 \%$ for $H$. pylori and $K$. pneumoniae, respectively (Naseer et al., 2021). In another report, Suresh et al., synthesized CuNPs applying a two-stage chemical reduction method, where Hydrazine Hydrate $(\mathrm{HH})$ was used as a reducing agent and Gum kondagogu extracts were used as a stabilizing agent. The SEM results revealed complete destruction of biofilm with destabilized cell masses in Klebsiella pneumoniae clinical isolates (ATCC 27736) treated with Gum kondagogu extract mediated CuNPs (Suresh et al., 2016).

Similarly, Punniyakotti et al., prepared copper nanoparticles (Cu NPs) using leaf extracts of Cardiospermum halicacabum and evaluated their antibiofilm efficacy against three clinical strains of $S$. aureus, E. coli, and $P$. aeruginosa. They demonstrated that at $100 \mu \mathrm{g} / \mathrm{ml}, \mathrm{Cu}$ NPs exhibited maximum antibiofilm activity with 79,78 , and $72 \%$ of biofilm inhibition in P. aeruginosa, E. coli, and S. aureus respectively (Punniyakotti et al., 2020). Similarly, Ali and his coworkers biosynthesized terpenoids entrapped copper oxide nanoparticles (ELE-CuONPs) prepared using Eucalyptus globulus (ELE) leaf extract. The results revealed that application of ELE-CuONPs in a dose ranging from 125 to $2000 \mu \mathrm{g} / \mathrm{ml}$, prevents biofilm development by $19.03 \pm 9 \%$ to $60.93 \pm 8 \%$, $44.41 \pm 7 \%$ to $70.75 \pm 8 \%$, and $34.41 \pm 7 \%$ to $62.29 \pm 8 \%$ in $P$. aeruginosa-621, E. coli-336, and MRSA-1, respectively, hence suggesting the promising potential of ELE-CuONPs as an antibiofilm therapeutic (Ali et al., 2019).

Interestingly, Erci and colleagues synthesized CuONPs by using various concentrations of aqueous leaf extract of Thymbra spicata to obtain Ts1CuONPs (40 ml plant extract) and Ts2CuONPs ( $80 \mathrm{ml}$ plant extract). Their data showed, at a dosage of $100 \mu \mathrm{g} / \mathrm{ml}$, the biofilm inhibition value for S.aureus was found to be $57.6 \pm 1.03 \%$ and $49.1 \pm 4.0 \%$ for Ts2CuONPs, Ts1CuONPs respectively (Erci et al., 2020). Accordingly, Cherian et al. manufactured CuONP via the one-pot green method by employing Cymbopogon citratus (CLE) leaf extract. The results revealed that the biofilm growth in MRSA-1 and E.coli was decreased to $49.0 \pm 3.1 \%$ and $33.0 \pm 3.2 \%$, respectively on exposure to CLE-CuONPs $(2000 \mu \mathrm{g} / \mathrm{ml})$. Further, CLSM data suggested superior antibiofilm properties against E.coli followed by $S$. aureus owing to the variation in their cell wall compositions (Cherian et al., 2020).

\section{Titanium Dioxide Nanoparticles}

Titanium oxide nanoparticles have a broad spectrum of applications like antibacterial, cosmetics, photocatalyst, 
wastewater treatment, and other medical fields. In fact, they are among the most extensively utilized nanoparticles attributed to their superior properties like non-toxic, stable, safe, and having surface activity (Al-Shabib et al., 2020). For instance, a study done by Narayanan et al. showed that $\mathrm{TiO}_{2} \mathrm{NPs}$ prevented the growth of bacterial pathogens via the generation of ROS, DNA, and cell membrane damage (Narayanan et al., 2021).

Accordingly, Al-Shabib and his colleagues fabricated $\mathrm{TiO}_{2}$ NPs using root extract of Withania somnifera and evaluated its antibiofilm efficacy. Their results showed that at a concentration below MIC $(0.5 \times \mathrm{MIC}), \mathrm{TiO}_{2}$ NPs exhibited a significant inhibitory effect on biofilm development (43-71\%) and mature biofilms (24-64\%) in pathogens like P. aeruginosa, E. coli, Listeria monocytogenes, MRSA, Serratia marcescens, and Candida albicans. Consequently, they inferred that excessive ROS generation followed by cell death could be the possible cause for the compromised biofilm development in $\mathrm{TiO}_{2} \mathrm{NP}$-treated pathogens (Al-Shabib et al., 2020).

Rajkumari and coworkers fabricated titanium dioxide nanoparticles $\left(\mathrm{TiO}_{2} \mathrm{NPs}\right)$ employing Aloe barbadensis leaf extract. Their results showed treatment of $P$. aeruginosa in biofilm mode with $\mathrm{TiO}_{2}$ NPS leads to a significant reduction in cell viability by $30.76 \pm 3.96 \%$. Further, the MIC value of $\mathrm{TiO}_{2}$ NPs imparted prominent antibiofilm activity against $P$. aeruginosa by hindering the adhesion of planktonic cells to the substratum (Rajkumari et al., 2019). Similarly, Achudhan and colleagues fabricated $\mathrm{TiO}_{2}$ NPs using twigs and bud extract of Ficus benghalensis, Azadirachta indica twigs, and Syzygium aromaticum and evaluated their antibacterial and antibiofilm efficacy against bacteria (Citrobacter freundii and Streptococcus mutans) and fungi (Candida albicans). The green synthesized $\mathrm{TiO}_{2}$ NPs inhibited biofilms of both the pathogens at a concentration of $100 \mu \mathrm{g} / \mathrm{ml}$ (Achudhan et al., 2020).

Interestingly, Dan et al., studied the antibiofilm activity of green synthesized titanium oxide using the leaf extract of Ocimum sanctum against $P$. aeruginosa, E. coli, S. aureus, and C. albicans. Their results showed the effective range of these NPs was between 250 and $650 \mu \mathrm{g} / \mathrm{ml}$ with a minimum effective dosage of $450 \mu \mathrm{g} / \mathrm{ml}$ against $E$. coli while for the remaining microbes $250 \mu \mathrm{g} / \mathrm{ml}$ was sufficient (Dan and Khan, 2019). Further, Jin et al. synthesized titanium dioxide nanostructures with the help of Rosa davurica leaf extract $\left(\mathrm{RDL}-\mathrm{TiO}_{2} \mathrm{NPs}\right)$ and evaluated its antibiofilm potential against different biofilm-producing bacteria. The minimum inhibitory concentration (MIC) of $\mathrm{RDL}-\mathrm{TiO}_{2} \mathrm{NPs}$ was $15.62 \mu \mathrm{g} / \mathrm{ml}$ for $S$. aureus and B. cereus, $62.5 \mu \mathrm{g} / \mathrm{ml}$ for S. enterica, and $31.25 \mu \mathrm{g} / \mathrm{ml}$ for E. coli. Also, the IC50 value for S. enterica and S. aureus were 158.75 and $76.84 \mu \mathrm{g} /$ $\mathrm{ml}$ respectively. Further, the TEM and CV assay results indicated that RDL-TiONPs prevented bacterial biofilm via cell wall and membrane rupture (Jin et al., 2021).

Zubair et al. evaluated the efficacy of green synthesized $\mathrm{TiO}_{2}$ NPs using leaf extract of Ochradenus arabicus to repress biofilm development and associated pathways in MRSA and MDR strains of $P$. aeruginosa isolated from foot ulcers. They studied that the constructed NPs reduced the biofilm formation by $22-70 \%$, inhibited EPS production and cell surface hydrophobicity at sub-MICs. Further, severe impairment of motility and a prominent decrease in alginate production was also observed in $P$. aeruginosa. Also, significant damage (51-63 percent) to the preformed biofilms of all tested strains was observed. Moreover, the treatment of bacterial cells with $\mathrm{TiO}_{2}$ NPs leads to increased ROS production which could be the possible reason for biofilm inhibition (Zubair et al., 2021).

\section{Iron Oxide Nanoparticles}

Recently, iron oxide nanoparticles (IONPs) have grabbed the limelight owing to their distinctive properties like higher surface area, superparamagnetism, surface-to-volume ratio, and simple separation procedure (Ali et al., 2016). These magnetic nanoparticles are known to impart antibacterial as well as antibiofilm properties via various mechanisms such as generation of ROS, electrostatic interaction with the cell membrane and its proteins, leading to physical damage and ultimately microbial death (Thukkaram et al., 2014). Further, the use of green materials for the synthesis of IONPs offers several advantages of low toxicity, biocompatibility for medical application, and eco-friendliness (Kanagasubbulakshmi and Kadirvelu, 2017).

In this regard, Ramalingam et al., constructed plant-assisted iron oxide nanoparticles utilizing leaf extract of grey mangrove Avicennia marina and studied its antibiofilm efficacy against marine pathogenic biofilm-forming bacteria. The designed NPs restricted the initial attachment and development of biofilm in biofilm-producing bacteria like Escherichia coli, Staphylococcus aureus, and Pseudomonas aeruginosa. Their results showed that nearly, $72 \%$ of quorum sensing factors of Pseudomonas aeruginosa, 63\% of $S$. aureus, and about $46 \%$ of E.coli were inhibited by $200 \mu \mathrm{g} / \mathrm{ml}$ of FeO-NPs. Moreover, the FeO-NPs reduced the hydrophobicity index of $P$. aeruginosa, $S$. aureus, and E. coli, from $19,15,16 \%$ to 11,11 , and $9 \%$ respectively. Also, the designed FeO-NPs prevented the formation of EPS in $P$. aeruginosa, S. aureus, and E. coli from $92,86,90 \%$, to 65,60 , and $69 \%$ respectively. Hence, they concluded that their designed biogenic FeO-NPs have the promising potential to be employed as an antibiofilm drug against pathogenic biofilmproducing microbes (Ramalingam et al., 2019).

Interestingly, the iron oxide nanoparticles synthesized using Thymbra spicata leaf extract imparted remarkable antibacterial properties against Salmonella typhimurium at a concentration of $100 \mu \mathrm{g} / \mathrm{ml}$, as studied by Erci et al. Further, the NPs inhibited the growth of $S$. aureus growth and inhibited biofilm growth by $25.9 \pm 3.36 \%$ at a concentration of $6.25 \mu \mathrm{g} / \mathrm{ml}$. Therefore, they concluded that green synthesized FeONPs using T. spicata could serve as a potential antibacterial and antibiofilm therapeutic candidate (Erci and Cakir-Koc, 2020).

Accordingly, Ansari and his coworkers have used an extract of a polyherbal ayurvedic drug formulation, Liv 52 for the synthesis of superparamagnetic maghemite nanoparticles (L52E- $\gamma-\mathrm{Fe}_{2} \mathrm{O}_{3}$ NPs). Their formulated NPs imparted superior antibacterial, antifungal as well as antibiofilm properties against MRSA, MDR $P$. aeruginosa, and C. albicans. Further, there was significant damage in the biofilm structure of $C$. albicans majorly due to intense cell wall damage and inhibited hyphal growth, as observed by scanning electron microscopy. Also, the molecular docking reports showed that there was an effective 
interaction between $\mathrm{Fe}_{2} \mathrm{O}_{3}$ NPs and cell wall peptidoglycan and mannoprotein which may lead to the cell wall and membrane damage (Ansari and Asiri, 2021).

\section{Cerium Oxide Nanoparticles}

Cerium oxide is a cubic-fluorite type of oxide which is regarded as the most prime among the rare earth oxides. Lately, $\mathrm{CeO}_{2}$ nanoparticles have been widely studied for a wide array of prospective applications in various fields including nanomedicine. Although, their antioxidant property has been well studied, but their antimicrobial and antibiofilm studies are still at it infancy with very limited studies (Kannan and Sundrarajan, 2014). Even though phytosynthesised $\mathrm{CeO}_{2}$ nanoparticles have shown promising antibiofilm potential, very little research work has been done.

In this regard, Ahmed and his coworkers employed fruit extract of Phoenix dactylifera for $\mathrm{CeO}_{2}$ and Ti-decorated $\mathrm{CeO}_{2}$ nanoparticles synthesis. They evaluated the antibiofilm efficacy of undoped and Ti-doped $\mathrm{CeO}_{2} \mathrm{NPs}$ against the $P$. aeruginosa developed biofilm. Their result revealed that undoped $\mathrm{CeO}_{2}$ nanoparticles prevented biofilm development by 11.0, 18.7, 31.3 , and $54.5 \%$ at a concentration of $125,250,500$, and $1000 \mu \mathrm{g} / \mathrm{ml}$ respectively. Similarly, $5 \%$ Ti-doped $\mathrm{CeO}_{2}$ nanoparticles inhibited biofilm formation by 10.9, 25.0, 34.2, and $57.4 \%$ at a concentrations of $125,250,500,1000 \mu \mathrm{g} / \mathrm{ml}$ respectively. Also, similar trends were observed with 10, 15, and $20 \% \quad \mathrm{Ti}-\mathrm{CeO}_{2}$ nanoparticles, with about $75 \%$ biofilm inhibition by $1000 \mu \mathrm{g} / \mathrm{ml}$ of $20 \% \quad \mathrm{Ti}-\mathrm{CeO}_{2}$ nanoparticles. Hence, their data indicated increasing the titanium doping concentration in $\mathrm{CeO}_{2}$ nanoparticles, enhances the antibiofilm activity, possibly because of the increased titanium ions release from the formulated nanocomposite (Ahmed et al., 2020).

Accordingly, Altaf et al. found that biosynthesized cerium oxide nanoparticles $\left(\mathrm{CeO}_{2}\right.$-NPs) prepared using Acorus calamus extract prevented the growth of bacterial biofilms by more than 75 percent. The confocal and electron microscopic data revealed that the application of $\mathrm{CeO}_{2}$-NPs prevented the development and colonization of the bacteria on solid surfaces. Moreover, dosedependent inhibition of preformed biofilms was also observed. There was a significant reduction in the exopolysaccharides (EPS) formation by test bacteria grown in $\mathrm{CeO}_{2}$-NPs supplemented culture media (Altaf et al., 2021).

Interestingly, Naidi et al., applied a green chemistry route for the synthesis of cerium oxide $\left(\mathrm{S}-\mathrm{CeO}_{2}\right)$ and 1,5 , and $10 \%$ tindoped cerium oxide nanoparticles ( $\mathrm{Sn}$-doped $\mathrm{CeO}_{2} \mathrm{NPs}$ ) using aqueous foliage extract of Pometia pinnata as reductant. The fabricated nanoparticles imparted substantial antibiofilm properties against $S$. aureus and Listeria monocytogenes at a concentration of $512 \mu \mathrm{g} / \mathrm{ml}$. The $1 \% \mathrm{Sn}$-doped $\mathrm{CeO}_{2} \mathrm{NPs}$ did not exhibit any antibiofilm activity while there was a significant antibiofilm activity imparted by the $10 \%$ Sn-doped $\mathrm{CeO}_{2}$ at a concentration of $512 \mu \mathrm{g} / \mathrm{ml}$ nearly similar to that of S-CeO 2 NPs (Naidi et al., 2021b). Similarly, in their other research work, they synthesized zirconium/tin-coated nanoparticles $\left(\mathrm{Zr} / \mathrm{Sn}-\mathrm{CeO}_{2}\right.$ NPs) using Pometia pinnata aqueous plant extract. All $\mathrm{Zr} / \mathrm{Sn}$ doped $\mathrm{CeO}_{2}$ NPs (1, 5, and 10\%) exhibited a concentrationdependent biofilm inhibition of L. monocytogenes, and only $10 \%$
$\mathrm{Zr} / \mathrm{Sn}$-dual doped-CeO $\mathrm{Ce}_{2} \mathrm{NPs}$ were found to impart antibiofilm activity against $S$. aureus at higher concentrations. The maximum biofilm inhibition was obtained at a concentration of $512 \mu \mathrm{g} / \mathrm{ml}$ for all of the synthesized nanoparticles i.e. $\mathrm{S}-\mathrm{CeO}_{2} \mathrm{NPs}$, and $\mathrm{Zr} /$ Sn-dual doped $\mathrm{CeO}_{2}$ NPs (Naidi et al., 2021a).

\section{Other Photosynthesized Nanoparticles}

Apart from the above-mentioned nanoparticles, few other phytosynthesized nanoparticles have also been remotely evaluated for their anti-biofilm properties. Regarding this, Saleem et al. employed a green route method for the synthesis of nickel oxide nanoparticles (NiO-NPs) by utilizing Eucalyptus globulus leaf extract as a bioreductant. They prominently established the antibiofilm and antibacterial efficacy of the synthesized NPs against ES $\beta \mathrm{L}(+)$ E. coli, P.aeruginosa, and S.aureus. Further, the SEM images of NiO-NPs treated with microbial cells revealed irregular shrinkage and distortion with prominent depression/indentations with the cells. Hence, their work indicated substantial antibiofilm and antibacterial activity of plant synthesized NiO-NPs (Saleem et al., 2017).

Similarly, Prakashkumar and his coworkers fabricated biocompatible palladium nanoparticles (NG@Pd NPs) using leaf extract of Orthosiphon stamineus and evaluated its antibiofilm and antibacterial properties. The results showed enhanced bactericidal activity of NG@Pd NPs against E. coli, $S$. aureus, and extended-spectrum beta-lactamase strain relative to the PdNPs alone. Moreover, the antibiofilm study demonstrated inhibition of biofilm development in a concentration-dependent manner in methicillin-sensitive Staphylococcus aureus strain with the IC50 value of $15.25 \pm$ $0.012 \mu \mathrm{g} / \mathrm{ml}$, whereas the Pd NPs exhibited reduced inhibitory effect ( $\mathrm{N}$ et al., 2021). Accordingly, Miglani and Tani-Ishii et al., constructed a biogenic selenium nanoparticle (SeNPs) using leaf extract of Psidium guajava with an aim to study its antimicrobial and antibiofilm properties against Enterococcus faecalis. Consequently, they evaluated the relative antibiofilm efficacy of five test groups namely distilled water (control), SeNPs $(1 \mathrm{mg} / \mathrm{ml})$, Calcium hydroxide $(1 \mathrm{mg} / \mathrm{ml}), 5.25 \%$ Sodium hypochlorite, and $2 \%$ Chlorhexidine gluconate. The result revealed that SeNPs were the most efficacious among others against the biofilm of E. faecalis (Miglani and Tani-Ishii, 2021).

\section{THE MECHANISM OF NANOPARTICLES EFFICACY AGAINST BIOFILM}

There are several studies done that support the superior efficacy of nanoparticles against biofilm-forming microorganisms. Due to their unique characteristics and small size, nanoparticles form a potential alternative to conventional antibiotics to treat biofilmrelated complications. Also, due to their higher surface area, they act as a suitable drug carrier by immobilizing drugs onto its surface thereby increasing its solubility and targeted delivery (Wang et al., 2017). There are numerous mechanisms via which nanoparticles impart their antimicrobial activity against these biofilm-forming pathogens which can be summarised under three categories i.e. 1) ROS (Reactive oxygen species) 


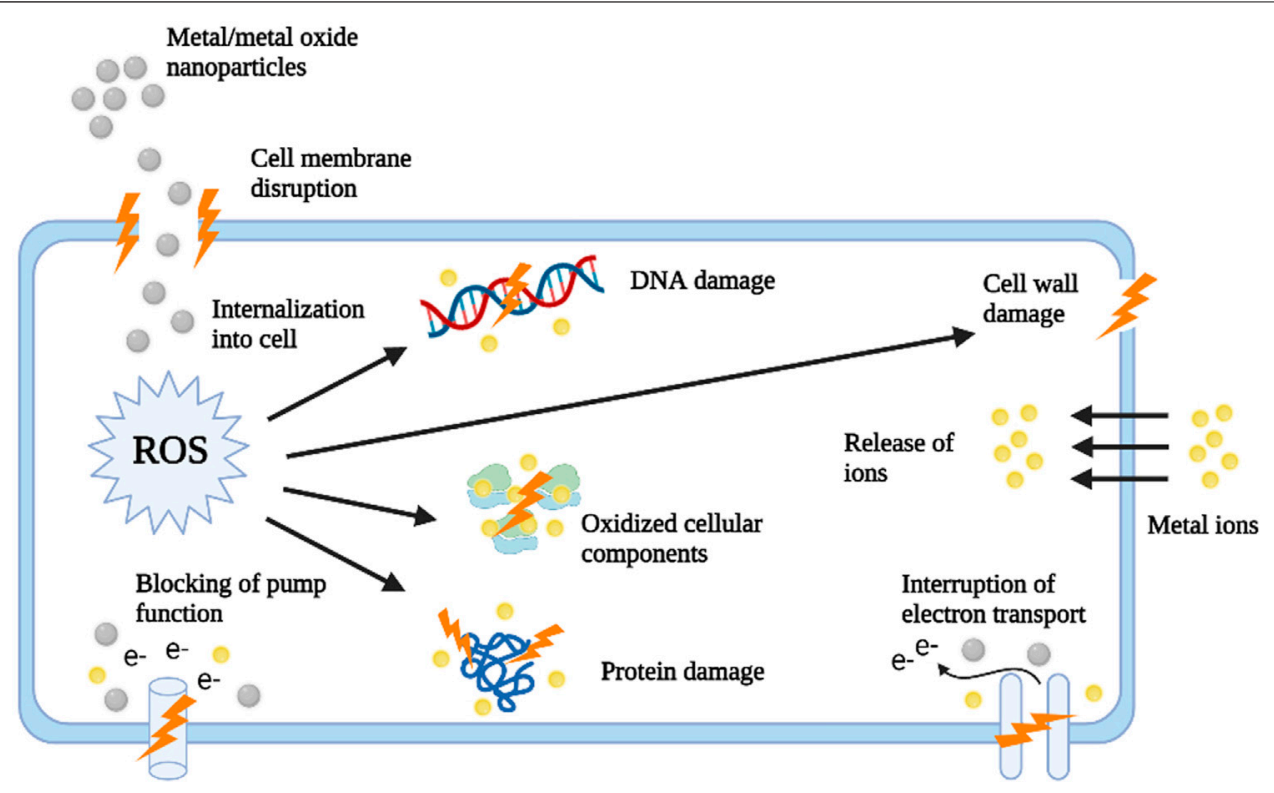

FIGURE 3 | Diagrammatic illustration representing of effects of various metal/metal oxide nanoparticles on a bacterial cell (Baptista et al., 2018).

induced oxidative stress, 2) release of metal ion thereby leading to alteration in enzyme activity, disruption of cell structures and protein denaturation, and 3) mechanical destruction of cell wall due to electrostatic interaction (Shkodenko et al., 2020) (Figure 3) (Baptista et al., 2018).

One of the prime antibacterial mechanisms of NPs includes induction of oxidative stress due to the production of ROS via electromagnetic irradiation of NPs (Dwivedi et al., 2014). Generally, ROS is formed due to aerobic respiration in bacteria which has an important role in cell death and survival modulation, cell signaling, and differentiation (Schieber and Chandel, 2014). But the extreme generation of ROS via NPs leads to imbalanced redox homeostasis thereby inducing oxidative stress which in turn affects the membrane lipids, proteins, and DNA structure. Various nanoparticles produce different ROS viz. hydrogen peroxide $\left(\mathrm{H}_{2} \mathrm{O}_{2}\right)$, superoxide $\left(\mathrm{O}_{2}{ }^{-}\right)$, and hydroxyl radical $(. \mathrm{OH})$ which is dependent on the chemical nature of the nanoparticles themselves (Dayem et al., 2017). It has been studied that while the endogenous antioxidants can neutralize the effects of superoxide $\left(\mathrm{O}_{2}^{-}\right)$and peroxide $\left(\mathrm{H}_{2} \mathrm{O}_{2}\right)$ radicals; the singlet oxygen and hydroxyl $(. \mathrm{OH})$ are fatal to bacterial cells causing acute death. Further, the production of ROS is directly proportional to the surface-to-volume ratio of the nanoparticles (L. Vega-Jiménez et al., 2019).

The metal ions of the metal oxide nanoparticles which are absorbed by the bacterial cell, can disturb the structure of the cell, alter the normal physiological functions and modify the activity of various proteins and enzymes via their interaction with functional groups of various intracellular nucleic acid and proteins such as sulfhydryl $(-\mathrm{SH})$, amino $(-\mathrm{NH})$, and carboxyl $(-\mathrm{COOH})$ groups. Metal ions also interact with DNA and modify its helical structure via cross-linking between as well as within DNA strands (Wang et al., 2017).
Non-oxidative mechanisms involve the interaction of the nanoparticles with the cell wall and cell membrane. The cell walls and membrane of bacteria function as protective shields against environmental damages. Cell membrane components provide various adsorption routes for the nanoparticles (Lesniak et al., 2013). The negatively charged bacterial cell wall interacts with the positively charged surface of NPs, via electrostatic interaction and Van der Waals interactions, this, in turn, causes alteration of cell membrane structure and permeability due to the accumulation of the nanoparticles onto the cell surface (Baptista et al., 2018).

Various research studies have indicated that NPs can obstruct biofilm formation via cell membrane disruption, thereby promoting bacterial death. Evidence from earlier reports showed that by interacting with EPS, eDNA, proteins, bacterial communication-quorum sensing (QS), and lipids of biofilm, NPs can disrupt biofilm stability (Qayyum and Khan, 2016). The surface chemistry and size of NPs play a central role in their antibiofilm activity as size regulates its effective penetration within the EPS matrix and surface chemistry guides the amount of interaction between NPs and EPS matrix. The strategies against biofilm inhibitions majorly aim to interfere with QS molecules. QS plays a vital role in bacterial pathogenesis including biofilm formation (Paluch et al., 2020). Therefore, the foundation for potential next-generation antivirulence treatments to be developed is the targeting and disruption of QS signaling systems and, as a result, biofilm formation.

Moreover, numerous nature-derived compounds are known to impart excellent antibiofilm activity but their clinical application is restricted due to their poor solubility and stability. Also, the free antibiotic fails to penetrate within these biofilms due to the EPS matrix. Further, the hydrophilic nature of commonly used antibiotics makes their cell penetration difficult. 
In this regard, NPs are known to function as an efficient delivery vehicle for the effective delivery of antibiotics/drugs within biofilm as well as within bacterial cells and thus increasing the efficacy of these drugs synergistically. Hence, in this way, NPs may be used to target bacterial biofilms in a variety of ways, including antibiotic-free and antibiotic-coated methods (Dos Santos Ramos et al., 2018).

\section{POTENTIAL ADVERSE EFFECTS OF METAL OXIDE NANOPARTICLES}

Despite displaying an array of significant applications in the field of medicine, nanoparticles can cause several prominent adverse effects on human health and the environment (Lanone and Boczkowski, 2006). Nanotoxicology is a sub-discipline of nanotechnology which encompasses the study of the toxicity of nanomaterials and evaluation of potential health risks associated with its application. The biomedical application of NPs for treating various diseases is a "double-edged sword," as the properties (small size, high reactivity, large surface area) which favor the beneficial application of nanoparticles, are also known to cause significant toxic effects (Donaldson et al., 2004). Other physicochemical properties like crystallinity, elemental composition, solubility, shape, charge, and surface derivatives may also affect the toxic potential of the nanoparticles (Dreher, 2004) (Nel et al., 2006). Moreover, agglomeration is one of the major obstructions in converting nanomaterials into medicine attributing to its potential toxic effects on various organs and organ systems (Andleeb et al., 2021). The small size makes the penetration of nanoparticles within minute capillaries feasible. Further, it has been reported that NPs with a size below $100 \mathrm{~nm}$ can penetrate cells, while sizes less than $40 \mathrm{~nm}$ can enter the nucleus, and sizes below $35 \mathrm{~nm}$ can cross the blood-brain barrier and enter the brain thereby may cause cellular damage. Further, studies have shown that these nanoparticles may exert cytotoxic effects in a cell type-, treatment time-, and dose-dependent manner (Brooking et al., 2001) (Arora et al., 2012).

When living organisms are exposed to different nanoparticles, it induces several toxic characteristics such as cytotoxicity, genotoxicity, and epigeneticity (Schrand et al., 2010) (Jennifer and Maciej, 2013). Further, The cyto-physiology of the cell may get affected by nanoparticles at various levels like genetic, organelle, or cellular levels which thereby add to the cytotoxicity, tumor development, and other side effects (Arora et al., 2012). Also, the application of NPs has made humans more prone to diseases like cancer, diabetes, allergies, bronchial asthma, inflammation, etc. Nanoparticles may impart their toxic effects on different cell structures via direct or indirect interactions with organic molecules of cells. Generation of excessive ROS and thereby disruption of intracellular redox homeostasis is considered to be one of the major mechanisms associated with NPs mediated macromolecule toxicity which leads to oxidative damage of macromolecules such as lipids, proteins, and nucleic acids (Radu et al., 2010) (De Planque et al., 2011). The oxidative DNA damage therefore may alter the interaction of DNA and methyltransferases thereby leading to DNA hypomethylation. In addition, nanoparticles may enhance the expression of different pro-inflammatory genes like TNF- $\alpha$, IL-1, IL-8, IL-6 via activation of the NF- $\kappa \mathrm{B}$ signaling pathway. Consequently, these molecular and cellular alterations lead to oxidative stress, followed by intense genotoxicity and subsequent programmed cell death (Luo et al., 2015). The alterations in hematological, oxidative enzymes, biochemical, and other histopathological criteria are regarded as the possible biomarkers for assessing the toxicity of nanoparticles in different living organisms (Kanwal et al., 2019).

Further, the enormous discharge of NPs as industrial nanowaste into the surrounding environment is proving to cause ecosystem imbalance and is hazardous to the indigenous living organisms. Also, the massive generation of NPs is distressing the food web of the ecosystem. NPs released in the atmosphere can gain excess into the plant via the stomatal openings of leaves whereas the NPs occurring in water or soil can selectively gain access via roots of the plants (Wang et al., 2013) (Tripathi et al., 2017). NPs may exert their toxicity on plants via excessive ROS production, DNA damage, a decline in plant biomass, reducing photosynthetic pigments and protein content, etc.; thereby impeding the growth and development of the plants (Zhu et al., 2019).

Also, due to their non-specific antimicrobial nature, the NPs not only kill pathogenic micro-organisms but also targets the symbiotic microbial population (Qayyum and Khan, 2016). Further, if the concentration of nanoparticles is not enough to eradicate biofilms but enough to cause mutation, then the nanoparticles can lead to the development of "super mutant" variants of bacteria (Shkodenko et al., 2020). The occurrence of NPs mutagenicity in bacterial biofilms plays an important role. The genetic material of lysed bacterial cells gets accumulated within the EPM, thereby leading to enhanced gene transfer frequency among bacterial cells (Kalishwaralal et al., 2010a). The genetic components like plasmid may contain genes for antibiotic resistance, therefore it can be assumed that nanoparticles may add to the development of antibiotic resistance bacteria (Durmus et al., 2013)

Since conventional chemical synthesis utilizes several hazardous compounds for nanoparticle synthesis, the developed nanoparticles tend to show undesirable side effects. An alternative to overcome this problem is to adopt eco-friendly methods to synthesis less toxic NPs i.e. biosynthesis of NPs. The utilization of the biological approach for the synthesis of NPs can also reduce the toxicity of nanoparticles. Studies have reported the biocompatible nature of several phytoconstituents (Singh J. et al., 2018). The toxic properties of the nanoparticles can also be regulated by incorporating free functional groups onto their surface. Moreover, toxicity can also be reduced by controlling the particle size of the designed metal nanoparticles (Singh and Lillard, 2009).

\section{CONCLUSION AND FUTURE PERSPECTIVE}

Biofilms being the most predominant mode of bacterial growth presents a significant challenge which is not been effectively 
encountered by traditional antimicrobial therapeutics. Further, its spatial and temporal diversity, both in terms of chemical and microbial structure, has added a degree of complexity to eradication methods. It has raised concerns that the usage of long-term antibiotics may lead to the occurrence of multidrugresistant strains. Biofilm infections are extremely resilient to present antimicrobial therapy and thereby is a severe matter of concern for the healthcare sector. As a consequence, the development of efficacious approaches to combat biofilms needs a multidisciplinary strategy to overcome various challenges offered by biofilms. Some of the pioneering and potential antibiofilm strategies include disruption of matured biofilms, isolation of quorum quenching compounds, an amalgamation of quorum quenching compounds with antibiotics, or a mixture of these mentioned techniques, which are still under investigation and did not reach clinical trial. Recently, the potential of nanoparticles as a promising antibiofilm agent has taken hold, attributed to its unique properties like small size, superior surface-area-to-volume ratio, and others. Among various routes employed for nanoparticles synthesis, the application of the green chemistry route has gained significant attention due to its several merits like eco-friendly, cost-effectiveness, low toxicity.

Even though the biological mode of synthesis has the advantages of green chemistry as mentioned in this literature, there are certain unsettled issues associated with it like, reproducibility of the synthesis process, consistency in particles shape and size, and the exact mechanism involved in the biosynthesis of nanoparticles. In the case of phytosynthesis, the mechanism of nanoparticles formation varies with different plant species. Further, the compositions of identical plants cultivated in various geographical locations or harvested in different climates might show variations in their active components. This, in turn, would influence the morphology of the synthesized nanoparticles as the shape and size of nanoparticles are majorly determined by the different compositions of bioactive molecules with predefined molecule sizes. This would lead to a fall in their commercial value and thereby make the marketing of phyto-synthesized nanoparticles more challenging. In the case of chemical synthesis, they have the advantage of regulated size and shape and which is why it is more preferred. Nonetheless, the beneficial facets outweigh the bad, which also pushes researchers to upgrade the ways of plant-mediated synthesis. Therefore, there is a need for a thorough understanding and evaluation of the plant-assisted synthesis mechanisms. This is a

\section{REFERENCES}

Abboud, Y., Saffaj, T., Chagraoui, A., El Bouari, A., Brouzi, K., Tanane, O., et al. (2014). Biosynthesis, Characterization and Antimicrobial Activity of Copper Oxide Nanoparticles (CONPs) Produced Using Brown Alga Extract (Bifurcaria Bifurcata). Appl. Nanosci. 4, 571-576. doi:10.1007/s13204-013-0233-x

Abdal Dayem, A., Hossain, M., Lee, S., Kim, K., Saha, S., Yang, G.-M., et al. (2017). The Role of Reactive Oxygen Species (ROS) in the Biological Activities of Metallic Nanoparticles. Ijms 18, 120. doi:10.3390/ ijms 18010120 poorly explored area and requires serious attention to fully explore and use the benefits of green synthesized metallic nanoparticles.

Various green synthesized metal/metal oxide nanoparticles such as $\mathrm{Ag}, \mathrm{Au}, \mathrm{ZnO}, \mathrm{CeO}_{2}, \mathrm{Fe}_{2} \mathrm{O}_{3}, \mathrm{CuO}, \mathrm{TiO}_{2}, \mathrm{Pd}$, Se, $\mathrm{NiO}$, have been assessed for their antibiofilm properties, among which phytosynthesized AgNPs are the most studied nanoparticles against biofilms. Yet, further research should be promoted to detect the activity of nanoparticles in industrial settings and their impact on the environment. Phytosynthesised nanoparticles have a promising future and can be applied in different biomedical fields involving nanoparticles like targeted drug delivery, fluorescent labeling in immunoassays, as antibacterial agents in bandages, and in destroying tumors via heating (hyperthermia). Even though there is increasing evidence regarding the promising potential of these green nanoparticles, it is obligatory to establish procedures to ensure their benign application to convert these preparations into reality as anti-biofilm therapeutics. It may be stated that, despite significant studies on nanoparticles across the world, many topics still need to be investigated further. Without them, the clinical introduction of these nano drugs can prove to be havoc for both mankind and the microbial community. A more structured and explicit study regarding NP's mechanism of action on planktonic cells, biofilms, mutagenicity, and genotoxicity may permit more targeted application thereby enhancing their effectiveness. Further, it is equally crucial to study the systemic in vivo effectiveness of these upcoming methodologies from the biomedical perspective. Specificity being the crucial factor for clinical applications, it is extremely important to differentiate between commensal and pathogenic bacteria and host tissue. Another key factor to be considered for investigation is how the nanoparticles are adapted in the biological setting, such as blood, and how these modifications will influence their effectiveness. Moreover, prospective directions should emphasize on the complete removal of biofilm by targeting the EPS matrix and the microbial cells simultaneously, thereby enhancing the therapeutic efficacy, while minimalizing toxic effects and development of resistance.

\section{AUTHOR CONTRIBUTIONS}

PD and SG are the first authors and they have contributed equally towards the literature survey and writing of the whole manuscript. BN is the corresponding author. She developed the concept, evaluated and verified the entire process from beginning to end, and corrected the final manuscript.

Abdel-Raouf, N., Al-Enazi, N. M., and Ibraheem, I. B. M. (2017). Green Biosynthesis of Gold Nanoparticles Using Galaxaura Elongata and Characterization of Their Antibacterial Activity. Arabian J. Chem. 10, S3029-S3039. doi:10.1016/j.arabjc.2013.11.044

AbdelRahim, K., Mahmoud, S. Y., Ali, A. M., Almaary, K. S., Mustafa, A. E.-Z. M. A., and Husseiny, S. M. (2017). Extracellular Biosynthesis of Silver Nanoparticles Using Rhizopus Stolonifer. Saudi J. Biol. Sci. 24, 208-216. doi:10.1016/j.sjbs.2016.02.025

Achudhan, D., Vijayakumar, S., Malaikozhundan, B., Divya, M., Jothirajan, M., Subbian, K., et al. (2020). The Antibacterial, Antibiofilm, Antifogging and Mosquitocidal Activities of Titanium Dioxide (TiO2) Nanoparticles green- 
synthesized Using Multiple Plants Extracts. J. Environ. Chem. Eng. 8, 104521. doi:10.1016/j.jece.2020.104521

Agnihotri, M., Joshi, S., Kumar, A. R., Zinjarde, S., and Kulkarni, S. (2009). Biosynthesis of Gold Nanoparticles by the Tropical Marine Yeast Yarrowia Lipolytica NCIM 3589. Mater. Lett. 63, 1231-1234. doi:10.1016/ j.matlet.2009.02.042

Ahmad, S., Tauseef, I., Haleem, K. S., Khan, K., Shahzad, M., Ali, M., et al. (2020). Synthesis of Silver Nanoparticles Using Leaves of Catharanthus Roseus and Their Antimicrobial Activity. Appl. Nanosci. 10, 4459-4464. doi:10.1007/ s13204-019-01221-z

Ahmed, A. S., Iqbal, A., Shafi, A., Qais, F. A., Ahamad, T., and Srivastava, S. (2020). Enhanced Removal of Crystal Violet Dye and Anti-biofilm Activity of Ti Doped CeO2 Nanoparticles Synthesized by Phoenix Dactylifera Mediated Green Method. J. Clust. Sci., 1-15. doi:10.1007/s10876-020-01925-1

Ahmed, S., Saifullah, M., Ahmad, B. L., and Ikram, S. (2016). Green Synthesis of Silver Nanoparticles Using Azadirachta indica Aqueous Leaf Extract. J. Radiat. Res. Appl. Sci. 9, 1-7. doi:10.1016/j.jrras.2015.06.006

Ajayi, E., and Afolayan, A. (2017). Green Synthesis, Characterization and Biological Activities of Silver Nanoparticles from Alkalinized Cymbopogon Citratus Stapf. Adv. Nat. Sci. Nanosci. Nanotechnol. 8, 015017. doi:10.1088/ 2043-6254/aa5cf7

Akhtar, M. S., Panwar, J., and Yun, Y.-S. (2013). Biogenic Synthesis of Metallic Nanoparticles by Plant Extracts. ACS Sustainable Chem. Eng. 1, 591-602. doi:10.1021/sc300118u

Al Aboody, M. S. (2019). Silver/silver Chloride (Ag/AgCl) Nanoparticles Synthesized from Azadirachta indica Lalex and its Antibiofilm Activity Against Fluconazole Resistant Candida tropicalis. Artif. Cell Nanomedicine, Biotechnol. 47, 2107-2113. doi:10.1080/21691401.2019.1620257

Al-Ansari, M., Alkubaisi, N., Gopinath, K., Karthika, V., Arumugam, A., and Govindarajan, M. (2019). Facile and Cost-Effective Ag Nanoparticles Fabricated by Lilium Lancifolium Leaf Extract: Antibacterial and Antibiofilm Potential. J. Clust. Sci. 30, 1081-1089. doi:10.1007/s10876-01901569-w

Al-Shabib, N. A., Husain, F. M., Ahmed, F., Khan, R. A., Ahmad, I., Alsharaeh, E., et al. (2016). Biogenic Synthesis of Zinc Oxide Nanostructures from Nigella Sativa Seed: Prospective Role as Food Packaging Material Inhibiting BroadSpectrum Quorum Sensing and Biofilm. Sci. Rep. 6, 36761. doi:10.1038/ srep36761

Al-Shabib, N. A., Husain, F. M., Hassan, I., Khan, M. S., Ahmed, F., Qais, F. A., et al. (2018). Biofabrication of Zinc Oxide Nanoparticle from Ochradenus baccatus Leaves: Broad-Spectrum Antibiofilm Activity, Protein Binding Studies, andIn VivoToxicity and Stress Studies. J. Nanomater. 2018, 1-14. doi:10.1155/2018/ 8612158

Al-Shabib, N. A., Husain, F. M., Qais, F. A., Ahmad, N., Khan, A., Alyousef, A. A., et al. (2020). Phyto-Mediated Synthesis of Porous Titanium Dioxide Nanoparticles from Withania Somnifera Root Extract: Broad-Spectrum Attenuation of Biofilm and Cytotoxic Properties Against HepG2 Cell Lines. Front. Microbiol. 11, 1680. doi:10.3389/fmicb.2020.01680

Alavi, M., Karimi, N., and Salimikia, I. (2019). Phytosynthesis of Zinc Oxide Nanoparticles and its Antibacterial, Antiquorum Sensing, Antimotility, and Antioxidant Capacities Against Multidrug Resistant Bacteria. J. Ind. Eng. Chem. 72, 457-473. doi:10.1016/j.jiec.2019.01.002

Ali, A., Zafar, H., Zia, M., ul Haq, I., Phull, A. R., Ali, J. S., et al. (2016). Synthesis, Characterization, Applications, and Challenges of Iron Oxide Nanoparticles. Nsa 9, 49-67. doi:10.2147/NSA.S99986

Ali, K., Ahmed, B., Ansari, S. M., Saquib, Q., Al-Khedhairy, A. A., Dwivedi, S., et al. (2019). Comparative In Situ ROS Mediated Killing of Bacteria with Bulk Analogue, Eucalyptus Leaf Extract (ELE)-capped and Bare Surface Copper Oxide Nanoparticles. Mater. Sci. Eng. C 100, 747-758. doi:10.1016/ j.msec.2019.03.012

Ali, K., Ahmed, B., Dwivedi, S., Saquib, Q., Al-Khedhairy, A. A., and Musarrat, J. (2015). Microwave Accelerated Green Synthesis of Stable Silver Nanoparticles with Eucalyptus Globulus Leaf Extract and Their Antibacterial and Antibiofilm Activity on Clinical Isolates. PLoS One 10, e0131178. doi:10.1371/journal.pone.0131178

Ali, S. G., Ansari, M. A., Alzohairy, M. A., Alomary, M. N., Alyahya, S., Jalal, M., et al. (2020). Biogenic Gold Nanoparticles as Potent Antibacterial and Antibiofilm Nano-Antibiotics Against Pseudomonas aeruginosa. Antibiotics 9, 100. doi:10.3390/antibiotics9030100
Ali, S. G., Ansari, M. A., Khan, H. M., Jalal, M., Mahdi, A. A., and Cameotra, S. S. (2018). Antibacterial and Antibiofilm Potential of Green Synthesized Silver Nanoparticles Against Imipenem Resistant Clinical Isolates of $P$. aeruginosa. BioNanoSci. 8, 544-553. doi:10.1007/s12668-018-0505-8

Almatroudi, A., Khadri, H., Azam, M., Rahmani, A. H., Al Khaleefah, F. K., Khateef, R., et al. (2020). Antibacterial, Antibiofilm and Anticancer Activity of Biologically Synthesized Silver Nanoparticles Using Seed Extract of Nigella Sativa. Processes 8, 388. doi:10.3390/PR8040388

Altaf, M., Manoharadas, S., and Zeyad, M. T. (2021). Green Synthesis of Cerium Oxide Nanoparticles Using Acorus calamus Extract and Their Antibiofilm Activity Against Bacterial Pathogens. Microsc. Res. Tech. 84, 1638-1648. doi:10.1002/jemt.23724

Amini, N., Amin, G., and Azar, Z. J. (2017). Green Synthesis of Silver Nanoparticles Using Avena Sativa L. Extract. Nanomedicine Res. J. doi:10.22034/ NMRJ.2017.23588

Amooaghaie, R., Saeri, M. R., and Azizi, M. (2015). Synthesis, Characterization and Biocompatibility of Silver Nanoparticles Synthesized from Nigella Sativa Leaf Extract in Comparison with Chemical Silver Nanoparticles. Ecotoxicology Environ. Saf. 120, 400-408. doi:10.1016/j.ecoenv.2015.06.025

Anastas, P. T., and Warner, J. C. (1998). Green Chemistry: Theory and Practice. New York, NY: Oxford Univ. Press.

Andleeb, A., Andleeb, A., Asghar, S., Zaman, G., Tariq, M., Mehmood, A., et al. (2021). A Systematic Review of Biosynthesized Metallic Nanoparticles as a Promising Anti-cancer-strategy. Cancers 13, 2818. doi:10.3390/ cancers 13112818

Ansari, M. A., and Asiri, S. M. M. (2021). Green Synthesis, Antimicrobial, Antibiofilm and Antitumor Activities of Superparamagnetic $\gamma$-Fe2O3 NPs and Their Molecular Docking Study with Cell wall Mannoproteins and Peptidoglycan. Int. J. Biol. Macromolecules 171, 44-58. doi:10.1016/ j.ijbiomac.2020.12.162

Arora, S., Rajwade, J. M., and Paknikar, K. M. (2012). Nanotoxicology and In Vitro Studies: The Need of the Hour. Toxicol. Appl. Pharmacol. 258, 151-165. doi:10.1016/j.taap.2011.11.010

Arsiya, F., Sayadi, M. H., and Sobhani, S. (2017). Green Synthesis of Palladium Nanoparticles Using Chlorella Vulgaris. Mater. Lett. 186, 113-115. doi:10.1016/ j.matlet.2016.09.101

Arya, G., Kumari, R. M., Sharma, N., Gupta, N., Kumar, A., Chatterjee, S., et al. (2019). Catalytic, Antibacterial and Antibiofilm Efficacy of Biosynthesised Silver Nanoparticles Using Prosopis Juliflora Leaf Extract Along with Their Wound Healing Potential. J. Photochem. Photobiol. B: Biol. 190, 50-58. doi:10.1016/j.jphotobiol.2018.11.005

Arya, G., Mankamna Kumari, R., Sharma, N., Chatterjee, S., Gupta, N., Kumar, A., et al. (2018). Evaluation of Antibiofilm and Catalytic Activity of Biogenic Silver Nanoparticles Synthesized from Acacia Nilotica Leaf Extract. Adv. Nat. Sci. Nanosci. Nanotechnol. 9, 045003. doi:10.1088/2043-6254/aae989

Ashajyothi, C., Harish, K. H., Dubey, N., and Chandrakanth, R. K. (2016). Antibiofilm Activity of Biogenic Copper and Zinc Oxide NanoparticlesAntimicrobials Collegiate Against Multiple Drug Resistant Bacteria: A Nanoscale Approach. J. Nanostruct Chem. 6, 329-341. doi:10.1007/s40097016-0205-2

Aziz, N., Fatma, T., Varma, A., and Prasad, R. (2014). Biogenic Synthesis of Silver Nanoparticles Using Scenedesmus Abundans and Evaluation of Their Antibacterial Activity. J. Nanoparticles 2014, 1-6. doi:10.1155/2014/689419

Azizi, S., Ahmad, M. B., Namvar, F., and Mohamad, R. (2014). Green Biosynthesis and Characterization of Zinc Oxide Nanoparticles Using Brown Marine Macroalga Sargassum Muticum Aqueous Extract. Mater. Lett. 116, 275-277. doi:10.1016/j.matlet.2013.11.038

Bai, H.-J., Yang, B.-S., Chai, C.-J., Yang, G.-E., Jia, W.-L., and Yi, Z.-B. (2011). Green Synthesis of Silver Nanoparticles Using Rhodobacter Sphaeroides. World J. Microbiol. Biotechnol. 27, 2723-2728. doi:10.1007/s11274-011-0747-x

Bakhtiari-Sardari, A., Mashreghi, M., Eshghi, H., Behnam-Rasouli, F., Lashani, E., and Shahnavaz, B. (2020). Comparative Evaluation of Silver Nanoparticles Biosynthesis by Two Cold-Tolerant Streptomyces Strains and Their Biological Activities. Biotechnol. Lett. 42, 1985-1999. doi:10.1007/s10529-020-02921-1

Banerjee, D., Shivapriya, P. M., Gautam, P. K., Misra, K., Sahoo, A. K., and Samanta, S. K. (2020). A Review on Basic Biology of Bacterial Biofilm Infections and Their Treatments by Nanotechnology-Based Approaches. Proc. Natl. Acad. Sci. India, Sect. B Biol. Sci. 90, 243-259. doi:10.1007/s40011-018-01065-7 
Baptista, P. V., McCusker, M. P., Carvalho, A., Ferreira, D. A., Mohan, N. M., Martins, M., et al. (2018). Nano-Strategies to Fight Multidrug Resistant Bacteria-"A Battle of the Titans". Front. Microbiol. 9, 1441. doi:10.3389/ fmicb.2018.01441

Barabadi, H., Mojab, F., Vahidi, H., Marashi, B., Talank, N., Hosseini, O., et al. (2021). Green Synthesis, Characterization, Antibacterial and Biofilm Inhibitory Activity of Silver Nanoparticles Compared to Commercial Silver Nanoparticles. Inorg. Chem. Commun. 129, 108647. doi:10.1016/j.inoche.2021.108647

Barbosa, D. B., Agostinho Hunt, A. M., Berretta, A., Rodrigues de Camargo, E., Gorup, L. F., Monteiro, D. R., et al. (2016). The Importance of Preventing and Controlling Biofilm in Wounds. Wound Healing Biomater. 2, 79-105. doi:10.1016/B978-1-78242-456-7.00004-0

Basumatari, M., Devi, R. R., Gupta, M. K., Gupta, S. K., Raul, P. K., Chatterjee, S., et al. (2021). Musa Balbisiana Colla Pseudostem Biowaste Mediated Zinc Oxide Nanoparticles: Their Antibiofilm and Antibacterial Potentiality. Curr. Res. Green Sustainable Chem. 4, 100048. doi:10.1016/j.crgsc.2020.100048

Bharathi, D., Vasantharaj, S., and Bhuvaneshwari, V. (2018). Green Synthesis of Silver Nanoparticles Using Cordia dichotoma Fruit Extract and its Enhanced Antibacterial, Anti-biofilm and Photo Catalytic Activity. Mater. Res. Express 5, 055404. doi:10.1088/2053-1591/aac2ef

Bhati-Kushwaha, H., and Malik, C. P. (2013). Biopotential of Verbesina Encelioides (Stem and Leaf Powders) in Silver Nanoparticle Fabrication. Turk J. Biol. 37, 645-654. doi:10.3906/biy-1212-7

Bhattacharya, P., Swarnakar, S., Ghosh, S., Majumdar, S., and Banerjee, S. (2019). Disinfection of Drinking Water via Algae Mediated Green Synthesized Copper Oxide Nanoparticles and its Toxicity Evaluation. J. Environ. Chem. Eng. 7, 102867. doi:10.1016/j.jece.2018.102867

Bhimba, B. V., Nath, N., and Sinha, P. (2011). Characterization and Antibacterial Analysis of Silver Nanoparticles Synthesized by the marine Fungi Hypocrea Lixii MV1 Isolated from Mangrove Sediment Soil. J. Pharm. Res. 4, 477-479.

Bhuyar, P., Rahim, M. H. A., Sundararaju, S., Ramaraj, R., Maniam, G. P., and Govindan, N. (2020). Synthesis of Silver Nanoparticles Using Marine Macroalgae Padina Sp. And its Antibacterial Activity towards Pathogenic Bacteria. Beni-suef Univ. J. Basic Appl. Sci. 9, 3. doi:10.1186/s43088-019-0031-y

Bing, W., Sun, H., Wang, F., Song, Y., and Ren, J. (2018). Hydrogen-producing Hyperthermophilic Bacteria Synthesized Size-Controllable Fine Gold Nanoparticles with Excellence for Eradicating Biofilm and Antibacterial Applications. J. Mater. Chem. B 6, 4602-4609. doi:10.1039/c8tb00549d

Borzenkov, M., Pallavicini, P., Taglietti, A., D’Alfonso, L., Collini, M., and Chirico, G. (2020). Photothermally Active Nanoparticles as a Promising Tool for Eliminating Bacteria and Biofilms. Beilstein J. Nanotechnol. 11, 1134-1146. doi:10.3762/BJNANO.11.98

Bosio, S., Leekha, S., Gamb, S. I., Wright, A. J., Terrell, C. L., and Miller, D. V. (2012). Mycobacterium Fortuitum Prosthetic Valve Endocarditis: A Case for the Pathogenetic Role of Biofilms. Cardiovasc. Pathol. 21, 361-364. doi:10.1016/ j.carpath.2011.11.001

Brackman, G., De Meyer, L., Nelis, H. J., and Coenye, T. (2013). Biofilm Inhibitory and Eradicating Activity of Wound Care Products Against Staphylococcus Aureus and Staphylococcus Epidermidis Biofilms in an In Vitro Chronic Wound Model. J. Appl. Microbiol. 114, 1833-1842. doi:10.1111/jam.12191

Brindha, S. G., Hansiya, V. S., Maheswari, P. U., and Geetha, N. (2020). A Comparative In Vitro Anti-biofilm Efficacy of Phytosynthesized Iron and Silver Nanoparticles. Int. J. App Pharm. 12, 72-76. doi:10.22159/ ijap.2020v12i1.35047

Brooking, J., Davis, S. S., and Illum, L. (2001). Transport of Nanoparticles Across the Rat Nasal Mucosa. J. Drug Target. 9, 267-279. doi:10.3109/ 10611860108997935

Bukhari, S. I., Hamed, M. M., Al-Agamy, M. H., Gazwi, H. S. S., Radwan, H. H., and Youssif, A. M. (2021). Biosynthesis of Copper Oxide Nanoparticles Using Streptomyces MHM38 and its Biological Applications. J. Nanomater. 2021, 1-16. doi:10.1155/2021/6693302

Camargo, L. F. A., Marra, A. R., Büchele, G. L., Sogayar, A. M. C., Cal, R. G. R., de Sousa, J. M. A., et al. (2009). Double-lumen Central Venous Catheters Impregnated with Chlorhexidine and Silver Sulfadiazine to Prevent Catheter Colonisation in the Intensive Care Unit Setting: A Prospective Randomised Study. J. Hosp. Infect. 72, 227-233. doi:10.1016/j.jhin.2009.03.018

Campbell, N. K., Fitzgerald, H. K., Fletcher, J. M., and Dunne, A. (2019). Plantderived Polyphenols Modulate Human Dendritic Cell Metabolism and
Immune Function via AMPK-dependent Induction of Heme Oxygenase-1. Front. Immunol. 10. doi:10.3389/fimmu.2019.00345

Castro-Longoria, E., Vilchis-Nestor, A. R., and Avalos-Borja, M. (2011). Biosynthesis of Silver, Gold and Bimetallic Nanoparticles Using the Filamentous Fungus Neurospora Crassa. Colloids Surf. B: Biointerfaces 83, 42-48. doi:10.1016/j.colsurfb.2010.10.035

Cauda, R. (2009). Candidaemia in Patients with an Inserted Medical Device. Drugs 69, 33-38. doi:10.2165/11315520-000000000-00000

Cherian, T., Ali, K., Fatima, S., Saquib, Q., Ansari, S. M., Alwathnani, H. A., et al. (2019). Myristica Fragrans Bio-Active Ester Functionalized ZnO Nanoparticles Exhibit Antibacterial and Antibiofilm Activities in Clinical Isolates. J. Microbiol. Methods 166, 105716. doi:10.1016/j.mimet.2019.105716

Cherian, T., Ali, K., Saquib, Q., Faisal, M., Wahab, R., and Musarrat, J. (2020). Cymbopogon Citratus Functionalized Green Synthesis of Cuo-Nanoparticles: Novel Prospects as Antibacterial and Antibiofilm Agents. Biomolecules 10, 169. doi:10.3390/biom10020169

Choi, J. S., Lee, J. W., Shin, U. C., Lee, M. W., Kim, D. J., and Kim, S. W. (2019). Inhibitory Activity of Silver Nanoparticles Synthesized Using Lycopersicon Esculentum Against Biofilm Formation in Candida Species. Nanomaterials 9, 1512. doi:10.3390/nano9111512

Chopade, B. A., Salunke, G. R., Ghosh, S., Santosh, R. J., Khade, S., Vashisth, P., et al. (2014). Rapid Efficient Synthesis and Characterization of Silver, Gold, and Bimetallic Nanoparticles from the Medicinal Plant Plumbago Zeylanica and Their Application in Biofilm Control. Ijn 9, 2635-2653. doi:10.2147/IJN.S59834

Ciofu, O., Tolker-Nielsen, T., Jensen, P. Ø., Wang, H., and Høiby, N. (2015). Antimicrobial Resistance, Respiratory Tract Infections and Role of Biofilms in Lung Infections in Cystic Fibrosis Patients. Adv. Drug Deliv. Rev. 85, 7-23. doi:10.1016/j.addr.2014.11.017

Costa, O. Y. A., Raaijmakers, J. M., and Kuramae, E. E. (2018). Microbial Extracellular Polymeric Substances: Ecological Function and Impact on Soil Aggregation. Front. Microbiol. 9, 1636. doi:10.3389/fmicb.2018.01636

Costerton, J. W., Geesey, G. G., and Cheng, K.-J. (1978). How Bacteria Stick. Sci. Am. 238, 86-95. doi:10.1038/scientificamerican0178-86

Cunha, F. A., Cunhada, M. d. C. S. O. C. S. O., da Frota, S. M., Mallmann, E. J. J., Freire, T. M., Costa, L. S., et al. (2018). Biogenic Synthesis of Multifunctional Silver Nanoparticles from Rhodotorula Glutinis and Rhodotorula Mucilaginosa: Antifungal, Catalytic and Cytotoxicity Activities. World J. Microbiol. Biotechnol. 34, 127. doi:10.1007/s11274-018-2514-8

Cusimano, M. G., Ardizzone, F., Nasillo, G., Gallo, M., Sfriso, A., MartinoChillura, D., et al. (2020). Biogenic Iron-Silver Nanoparticles Inhibit Bacterial Biofilm Formation Due to Ag+ Release as Determined by a Novel Phycoerythrin-Based Assay. Appl. Microbiol. Biotechnol. 104, 6325-6336. doi:10.1007/s00253-020-10686-w

Dan, S. O., and Khan, S. H. (2019). Green Coalescence and Characterization of Tio2 Nanoparticles and Evaluation of its Antibiofilm Activity. Rjc 12, 2252-2259. doi:10.31788/RJC.2019.1245341

Daraio, C., and Jin, S. (2012). Synthesis and Patterning Methods for Nanostructures Useful for Biological Applications, Nanotechnology for Biology and Medicine, 27, 44. doi:10.1007/978-0-387-31296-5_2

de Aragão, A. P., de Oliveira, T. M., Quelemes, P. V., Perfeito, M. L. G., Araújo, M. C., Santiago, J. d. A. S., et al. (2019). Green Synthesis of Silver Nanoparticles Using the Seaweed Gracilaria Birdiae and Their Antibacterial Activity. Arabian J. Chem. 12, 4182-4188. doi:10.1016/j.arabjc.2016.04.014

De Planque, M. R. R., Aghdaei, S., Roose, T., and Morgan, H. (2011). Electrophysiological Characterization of Membrane Disruption by Nanoparticles. ACS Nano 5, 3599-3606. doi:10.1021/nn103320j

DeAntonio, R., Yarzabal, J.-P., Cruz, J. P., Schmidt, J. E., and Kleijnen, J. (2016). Epidemiology of Otitis Media in Children from Developing Countries: A Systematic Review. Int. J. Pediatr. Otorhinolaryngol. 85, 65-74. doi:10.1016/ j.ijporl.2016.03.032

Del Pozo, J. L., and Patel, R. (2009). Infection Associated with Prosthetic Joints. N. Engl. J. Med. 361, 787-794. doi:10.1056/nejmcp0905029

Dhand, C., Dwivedi, N., Loh, X. J., Jie Ying, A. N., Verma, N. K., Beuerman, R. W., et al. (2015). Methods and Strategies for the Synthesis of Diverse Nanoparticles and Their Applications: A Comprehensive Overview. RSC Adv. 5, 105003-105037. doi:10.1039/c5ra19388e

Dhandapani, P., Maruthamuthu, S., and Rajagopal, G. (2012). Bio-mediated Synthesis of $\mathrm{TiO} 2$ Nanoparticles and its Photocatalytic Effect on Aquatic 
Biofilm. J. Photochem. Photobiol. B: Biol. 110, 43-49. doi:10.1016/ j.jphotobiol.2012.03.003

Dhillon, G. S., Brar, S. K., Kaur, S., and Verma, M. (2012). Green Approach for Nanoparticle Biosynthesis by Fungi: Current Trends and Applications. Crit. Rev. Biotechnol. 32, 49-73. doi:10.3109/07388551.2010.550568

Donaldson, K., Stone, V., Tran, C. L., Kreyling, W., and Borm, P. J. A. (2004). Nanotoxicology. Occup. Environ. Med. 61, 727-728. doi:10.1136/oem.2004.013243

Donlan, R. (2001). Biofilms and Device-Associated Infections. Emerg. Infect. Dis. 7, 277-281. doi:10.3201/eid0702.010226

Donlan, R. M. (2002). Biofilms: Microbial Life on Surfaces. Emerg. Infect. Dis. 8, 881-890. doi:10.3201/eid0809.020063

Donlan, R. M., and Costerton, J. W. (2002). Biofilms: Survival Mechanisms of Clinically Relevant Microorganisms. Clin. Microbiol. Rev. 15, 167-193. doi:10.1128/CMR.15.2.167-193.2002

Dos Santos Ramos, M. A., Da Silva, P., Spósito, L., De Toledo, L., Bonifácio, B., Rodero, C. F., et al. (2018). Nanotechnology-based Drug Delivery Systems for Control of Microbial Biofilms: A Review. Ijn 13, 1179-1213. doi:10.2147/ IJN.S146195

Doğan Çalhan, S., and Gündoğan, M. (2020). Biosynthesis of Silver Nanoparticles Using Onosmasericeum Willd. And Evaluation of Their Catalytic Properties and Antibacterial and Cytotoxic Activity. Turk J. Chem. 44, 1587-1600. doi:10.3906/kim-2007-1

Doğan, S. Ş., and Kocabaş, A. (2020). Green Synthesis of ZnO Nanoparticles with Veronica Multifida and Their Antibiofilm Activity. Hum. Exp. Toxicol. 39, 319-327. doi:10.1177/0960327119888270

Dreher, K. L. (2004). Health and Environmental Impact of Nanotechnology: Toxicological Assessment of Manufactured Nanoparticles. Toxicol. Sci. 77, 3-5. doi:10.1093/toxsci/kfh041

Drummer, S., Madzimbamuto, T., and Chowdhury, M. (2021). Green Synthesis of Transition-Metal Nanoparticles and Their Oxides: A Review. Materials 14, 2700. doi: $10.3390 / \mathrm{ma} 14112700$

Du, J., Singh, H., and Yi, T.-H. (2016). Antibacterial, Anti-biofilm and Anticancer Potentials of green Synthesized Silver Nanoparticles Using Benzoin Gum (Styrax Benzoin) Extract. Bioproc. Biosyst. Eng. 39, 1923-1931. doi:10.1007/ s00449-016-1666-x

Duan, H., Wang, D., and Li, Y. (2015). Green Chemistry for Nanoparticle Synthesis. Chem. Soc. Rev. 44, 5778-5792. doi:10.1039/c4cs00363b

Dunne, W. M. (2002). Bacterial Adhesion: Seen Any Good Biofilms Lately? Clin. Microbiol. Rev. 15, 155-166. doi:10.1128/CMR.15.2.155-166.2002

Durmus, N. G., Taylor, E. N., Kummer, K. M., and Webster, T. J. (2013). Enhanced Efficacy of Superparamagnetic Iron Oxide Nanoparticles Against AntibioticResistant Biofilms in the Presence of Metabolites. Adv. Mater. 25, 5706-5713. doi:10.1002/adma.201302627

Dwivedi, A. D., and Gopal, K. (2010). Biosynthesis of Silver and Gold Nanoparticles Using Chenopodium album Leaf Extract. Colloids Surf. A: Physicochemical Eng. Aspects 369, 27-33. doi:10.1016/j.colsurfa.2010.07.020

Dwivedi, S., Wahab, R., Khan, F., Mishra, Y. K., Musarrat, J., and Al-Khedhairy, A. A. (2014). Reactive Oxygen Species Mediated Bacterial Biofilm Inhibition via Zinc Oxide Nanoparticles and Their Statistical Determination. PLoS One 9, e111289. doi:10.1371/journal.pone.0111289

Dwivedy, A. K., Upadhyay, N., Asawa, S., Kumar, M., Prakash, B., and Dubey, N. K. (2019). Therapeutic Potential of Plant-Based Metal Nanoparticles. Algae Microorg. Concepts Controversies 2, 169-196. doi:10.1016/B978-0-12811488-9.00009-3

Elumalai, E. K., Prasad, T. N. V. K. V., Hemachandran, J., Viviyan Therasa, S., Thirumalai, T., and David, E. (2010). Extracellular Synthesis of Silver Nanoparticles Using Leaves of Euphorbia Hirta and Their Antibacterial Activities. J. Pharm. Sci. Res. 2, 549-554.

Emanuel, V., Adrian, V., and Diana, P. (2010). Microbial Biofilm Formation under the Influence of Various Physical-Chemical Factors. Biotechnol. Biotechnological Equipment 24, 1993-1996. doi:10.2478/V10133-010-0056-9

Erci, F., and Cakir-Koc, R. (2020). Rapid green Synthesis of Noncytotoxic Iron Oxide Nanoparticles Using Aqueous Leaf Extract of Thymbra Spicata and Evaluation of Their Antibacterial, Antibiofilm, and Antioxidant Activity. Inorg. Nano-Metal Chem. 51, 683-692. doi:10.1080/ 24701556.2020.1802754

Erci, F., Cakir-Koc, R., Yontem, M., and Torlak, E. (2020). Synthesis of Biologically Active Copper Oxide Nanoparticles as Promising Novel Antibacterial-
Antibiofilm Agents. Prep. Biochem. Biotechnol. 50, 538-548. doi:10.1080/ 10826068.2019.1711393

Erci, F., and Torlak, E. (2019). Antimicrobial and Antibiofilm Activity of Green Synthesized Silver Nanoparticles by Using Aqueous Leaf Extract of Thymus Serpyllum. Sak. Univ. J. Sci. 23, 1. doi:10.16984/saufenbilder.445146

Fakhari, S., Jamzad, M., and Kabiri Fard, H. (2019). Green Synthesis of Zinc Oxide Nanoparticles: A Comparison. Green. Chem. Lett. Rev. 12, 19-24. doi:10.1080/ 17518253.2018.1547925

Fesharaki, P. J., Nazari, P., Shakibaie, M., Rezaie, S., Banoee, M., Abdollahi, M., et al. (2010). Biosynthesis of Selenium Nanoparticles Using Klebsiella pneumoniae and Their Recovery by a Simple Sterilization Process. Braz. J. Microbiol. 41, 461-466. doi:10.1590/S1517-83822010000200028

Fijan, S., Frauwallner, A., Langerholc, T., Krebs, B., ter Haar, J. A., Heschl, A., et al. (2019). Efficacy of Using Probiotics with Antagonistic Activity Against Pathogens of Wound Infections: An Integrative Review of Literature. Biomed. Res. Int. 2019, 1-21. doi:10.1155/2019/7585486

Flemming, H.-C., Wingender, J., Szewzyk, U., Steinberg, P., Rice, S. A., and Kjelleberg, S. (2016). Biofilms: An Emergent Form of Bacterial Life. Nat. Rev. Microbiol. 14, 563-575. doi:10.1038/nrmicro.2016.94

Foroohimanjili, F., Mirzaie, A., Hamdi, S. M. M., Noorbazargan, H., Hedayati Ch, M., Dolatabadi, A., et al. (2020). Antibacterial, Antibiofilm, and Antiquorum Sensing Activities of Phytosynthesized Silver Nanoparticles Fabricated from Mespilus Germanica Extract against Multidrug Resistance of Klebsiella pneumoniae Clinical Strains. J. Basic Microbiol. 60, 216-230. doi:10.1002/ jobm.201900511

Gahlawat, G., and Choudhury, A. R. (2019). A Review on the Biosynthesis of Metal and Metal Salt Nanoparticles by Microbes. RSC Adv. 9, 12944-12967. doi:10.1039/c8ra10483b

Galedari, S., and Teimouri, M. (2020). Study of the Physicochemical Properties and Anti-biofilm Effects of Synthesized Zinc Oxide Nanoparticles Using Artemisia Plant. Int. J. Basic Sci. Med. 5, 101-107. doi:10.34172/ ijbsm.2020.18

Galié, S., García-Gutiérrez, C., Miguélez, E. M., Villar, C. J., and Lombó, F. (2018). Biofilms in the Food Industry: Health Aspects and Control Methods. Front. Microbiol. 9, 898. doi:10.3389/fmicb.2018.00898

Gardea-Torresdey, J. L., Gomez, E., Peralta-Videa, J. R., Parsons, J. G., Troiani, H., and Jose-Yacaman, M. (2003). Alfalfa Sprouts: A Natural Source for the Synthesis of Silver Nanoparticles. Langmuir 19, 1357-1361. doi:10.1021/ la020835i

Gebreyohannes, G., Nyerere, A., Bii, C., and Sbhatu, D. B. (2019). Challenges of Intervention, Treatment, and Antibiotic Resistance of Biofilm-Forming Microorganisms. Heliyon 5, e02192. doi:10.1016/j.heliyon.2019.e02192

Gong, J., Song, X., Gao, Y., Gong, S., Wang, Y., and Han, J. (2018). Microbiological Synthesis of Zinc Sulfide Nanoparticles Using Desulfovibrio Desulfuricans. Inorg. Nano-Metal Chem. 48, 96-102. doi:10.1080/15533174.2016.1216451

Goodrich, L. R. (2019). Osteomyelitis. In Equine Fracture Repair, 851-873. doi:10.1002/9781119108757.ch48

Govindappa, M., Tejashree, S., Thanuja, V., Hemashekhar, B., Srinivas, C., Nasif, O., et al. (2021). Pomegranate Fruit Fleshy Pericarp Mediated Silver Nanoparticles Possessing Antimicrobial, Antibiofilm Formation, Antioxidant, Biocompatibility and Anticancer Activity. J. Drug Deliv. Sci. Technology 61, 102289. doi:10.1016/j.jddst.2020.102289

Gowramma, B., Keerthi, U., Rafi, M., and Muralidhara Rao, D. (2015). Biogenic Silver Nanoparticles Production and Characterization from Native Stain of Corynebacterium Species and its Antimicrobial Activity. 3 Biotech. 5, 195-201. doi:10.1007/s13205-014-0210-4

Gudkov, S. V., Burmistrov, D. E., Serov, D. A., Rebezov, M. B., Semenova, A. A., and Lisitsyn, A. B. (2021). A Mini Review of Antibacterial Properties of $\mathrm{ZnO}$ Nanoparticles. Front. Phys. 9, 641481. doi:10.3389/fphy.2021.641481

Guiglia, R., Musciotto, A., Compilato, D., Procaccini, M., Russo, L., Ciavarella, D., et al. (2010). Aging and Oral Health: Effects in Hard and Soft Tissues. Cpd 16, 619-630. doi:10.2174/138161210790883813

Gupta, K., Barua, S., Hazarika, S. N., Manhar, A. K., Nath, D., Karak, N., et al. (2014a). Green Silver Nanoparticles: Enhanced Antimicrobial and Antibiofilm Activity with Effects on DNA Replication and Cell Cytotoxicity. RSC Adv. 4, 52845-52855. doi:10.1039/c4ra08791g

Gupta, K., Hazarika, S. N., Saikia, D., Namsa, N. D., and Mandal, M. (2014b). One Step Green Synthesis and Anti-microbial and Anti-biofilm Properties of 
Psidium Guajava L. Leaf Extract-Mediated Silver Nanoparticles. Mater. Lett. 125, 67-70. doi:10.1016/J.MATLET.2014.03.134

Gurunathan, S., Kalishwaralal, K., Vaidyanathan, R., Venkataraman, D., Pandian, S. R. K., Muniyandi, J., et al. (2009). Biosynthesis, Purification and Characterization of Silver Nanoparticles Using Escherichia coli. Colloids Surf. B: Biointerfaces 74, 328-335. doi:10.1016/j.colsurfb.2009.07.048

H Madkour, L. (2017). Ecofriendly green Biosynthesized of Metallic Nanoparticles: Bio-Reduction Mechanism, Characterization and Pharmaceutical Applications in Biotechnology Industry. Glob. Drugs Therap 3, 1-11. doi:10.15761/ gdt.1000144

Hall-Stoodley, L., and Stoodley, P. (2009). Evolving Concepts in Biofilm Infections. Cell. Microbiol 11, 1034-1043. doi:10.1111/j.1462-5822.2009.01323.x

He, S., Guo, Z., Zhang, Y., Zhang, S., Wang, J., and Gu, N. (2007). Biosynthesis of Gold Nanoparticles Using the Bacteria Rhodopseudomonas Capsulata. Mater. Lett. 61, 3984-3987. doi:10.1016/j.matlet.2007.01.018

Hector, A., Frey, N., and Hartl, D. (2016). Update on Host-Pathogen Interactions in Cystic Fibrosis Lung Disease. Mol. Cel. Pediatr. 3. doi:10.1186/s40348-0160039-5

Homøe, P., Bjarnsholt, T., Wessman, M., Sørensen, H. C. F., and Johansen, H. K. (2009). Morphological Evidence of Biofilm Formation in Greenlanders with Chronic Suppurative Otitis media. Eur. Arch. Otorhinolaryngol. 266, 1533-1538. doi:10.1007/s00405-009-0940-9

Hu, D., Si, W., Qin, W., Jiao, J., Li, X., Gu, X., et al. (2019). Cucurbita Pepo Leaf Extract Induced Synthesis of Zinc Oxide Nanoparticles, Characterization for the Treatment of Femoral Fracture. J. Photochem. Photobiol. B: Biol. 195, 12-16. doi:10.1016/j.jphotobiol.2019.04.001

Hulkoti, N. I., and Taranath, T. C. (2014). Biosynthesis of Nanoparticles Using Microbes-A Review. Colloids Surf. B: Biointerfaces 121, 474-483. doi:10.1016/ j.colsurfb.2014.05.027

Husseiny, M. I., El-Aziz, M. A., Badr, Y., and Mahmoud, M. A. (2007). Biosynthesis of Gold Nanoparticles Using Pseudomonas aeruginosa. Spectrochimica Acta A: Mol. Biomol. Spectrosc. 67, 1003-1006. doi:10.1016/j.saa.2006.09.028

Iravani, S., and Zolfaghari, B. (2013). Green Synthesis of Silver Nanoparticles UsingPinus eldaricaBark Extract. Biomed. Res. Int. 2013, 1-5. doi:10.1155/ 2013/639725

Isaac, R. S. R., Sakthivel, G., and Murthy, C. (2013). Green Synthesis of Gold and Silver Nanoparticles UsingAverrhoa bilimbiFruit Extract. J. Nanotechnology 2013, 1-6. doi:10.1155/2013/906592

Ishwarya, R., Vaseeharan, B., Kalyani, S., Banumathi, B., Govindarajan, M., Alharbi, N. S., et al. (2018). Facile Green Synthesis of Zinc Oxide Nanoparticles Using Ulva Lactuca Seaweed Extract and Evaluation of Their Photocatalytic, Antibiofilm and Insecticidal Activity. J. Photochem. Photobiol. B: Biol. 178, 249-258. doi:10.1016/j.jphotobiol.2017.11.006

İspirli, H., Sagdic, O., and Dertli, E. (2021). Synthesis of Silver Nanoparticles Prepared with a Dextran-type Exopolysaccharide from Weissella Cibaria MED17 with Antimicrobial Functions. Prep. Biochem. Biotechnol. 51, 112-119. doi:10.1080/10826068.2020.1795673

Jadoun, S., Arif, R., Jangid, N. K., and Meena, R. K. (2021). Green Synthesis of Nanoparticles Using Plant Extracts: A Review. Environ. Chem. Lett. 19, 355-374. doi:10.1007/s10311-020-01074-x

Jain, N., Bhargava, A., Majumdar, S., Tarafdar, J. C., and Panwar, J. (2011). Extracellular Biosynthesis and Characterization of Silver Nanoparticles Using Aspergillus flavusNJP08: A Mechanism Perspective. Nanoscale 3, 635-641. doi:10.1039/c0nr00656d

Jamal, M., Ahmad, W., Andleeb, S., Jalil, F., Imran, M., Nawaz, M. A., et al. (2018). Bacterial Biofilm and Associated Infections. J. Chin. Med. Assoc. 81, 7-11. doi:10.1016/j.jcma.2017.07.012

Jamkhande, P. G., Ghule, N. W., Bamer, A. H., and Kalaskar, M. G. (2019). Metal Nanoparticles Synthesis: An Overview on Methods of Preparation, Advantages and Disadvantages, and Applications. J. Drug Deliv. Sci. Technology 53, 101174. doi:10.1016/j.jddst.2019.101174

Janbon, G., Quintin, J., Lanternier, F., and d'Enfert, C. (2019). Studying Fungal Pathogens of Humans and Fungal Infections: Fungal Diversity and Diversity of Approaches. Genes Immun. 20, 403-414. doi:10.1038/s41435-019-0071-2

Jayabalan, J., Mani, G., Krishnan, N., Pernabas, J., Devadoss, J. M., and Jang, H. T. (2019). Green Biogenic Synthesis of Zinc Oxide Nanoparticles Using Pseudomonas Putida Culture and its In Vitro Antibacterial and Anti- biofilm Activity. Biocatal. Agric. Biotechnol. 21, 101327. doi:10.1016/ j.bcab.2019.101327

Jayaseelan, C., Rahuman, A. A., Roopan, S. M., Kirthi, A. V., Venkatesan, J., Kim, S.-K., et al. (2013). Biological Approach to Synthesize TiO2 Nanoparticles Using Aeromonas Hydrophila and its Antibacterial Activity. Spectrochimica Acta Part A: Mol. Biomol. Spectrosc. 107, 82-89. doi:10.1016/j.saa.2012.12.083

Jeevanandam, J., Chan, Y. S., and Danquah, M. K. (2016). Biosynthesis of Metal and Metal Oxide Nanoparticles. ChemBioEng Rev. 3, 55-67. doi:10.1002/ cben. 201500018

Jena, J., Pradhan, N., Prasad Dash, B., Behari Sukla, L., and kumar Panda Affiliations, P. (2013). Biosynthesis and Characterization of Silver Nanoparticles Using Microalga Chlorococcum Humicola and its Antibacterial Activity. Int. J. Nanomater. Biostructures. 3, 1-8.

Jennifer, M., and Maciej, W. (2013). Nanoparticle Technology as a Double-Edged Sword: Cytotoxic, Genotoxic and Epigenetic Effects on Living Cells. Jbnb 04, 53-63. doi:10.4236/jbnb.2013.41008

Jin, Y., Li, B., Saravanakumar, K., Hu, X., and Wang, M.-H. (2021). Phytogenic Titanium Dioxide (TiO2) Nanoparticles Derived from Rosa Davurica with Anti-bacterial and Anti-biofilm Activities. J. Clust. Sci., 1-9. doi:10.1007/ s10876-021-02024-5

Jo, J. H., Singh, P., Kim, Y. J., Wang, C., Mathiyalagan, R., Jin, C.-G., et al. (2016). Pseudomonas Deceptionensis DC5-mediated Synthesis of Extracellular Silver Nanoparticles. Artif. Cell Nanomedicine, Biotechnol. 44, 1576-1581. doi:10.3109/21691401.2015.1068792

Joshi, N., Jain, N., Pathak, A., Singh, J., Prasad, R., and Upadhyaya, C. P. (2018). Biosynthesis of Silver Nanoparticles Using Carissa Carandas Berries and its Potential Antibacterial Activities. J. Sol-gel Sci. Technol. 86, 682-689. doi:10.1007/s10971-018-4666-2

Kalabegishvili, T., Kirkesali, E., Rcheulishvili, A., Ginturi, E., Murusidze, I., Kuchava, N., et al. (2012). Synthesis of Gold Nanoparticles by Blue-Green Algae Spirulina Platensis. Adv. Sci. Eng. Med.

Kaler, A., Nankar, R., Bhattacharyya, M. S., and Banerjee, U. C. (2011). Extracellular Biosynthesis of Silver Nanoparticles Using Aqueous Extract of candida Viswanathii. $j$ bionanosci 5, 53-58. doi:10.1166/jbns.2011.1040

Kalishwaralal, K., BarathManiKanth, S., Pandian, S. R. K., Deepak, V., and Gurunathan, S. (2010a). Silver Nanoparticles Impede the Biofilm Formation by Pseudomonas aeruginosa and Staphylococcus Epidermidis. Colloids Surf. B: Biointerfaces 79, 340-344. doi:10.1016/j.colsurfb.2010.04.014

Kalishwaralal, K., Deepak, V., Ram Kumar Pandian, S., Kottaisamy, M., BarathManiKanth, S., Kartikeyan, B., et al. (2010b). Biosynthesis of Silver and Gold Nanoparticles Using Brevibacterium Casei. Colloids Surf. B: Biointerfaces 77, 257-262. doi:10.1016/j.colsurfb.2010.02.007

Kamble, S. P., and Shinde, K. D. (2018). Anti-biofilm Activity Against GramPositive Bacteria by Biologically Synthesized Silver Nanoparticles Using Curcuma Longa. Pnt 6, 165-170. doi:10.2174/ 2211738506666180629142001

Kanagasubbulakshmi, S., and Kadirvelu, K. (2017). Green Synthesis of Iron Oxide Nanoparticles Using Lagenaria Siceraria and Evaluation of its Antimicrobial Activity. Def. Life Sc. Jl. 2, 422. doi:10.14429/dlsj.2.12277

Kannan, S. K., and Sundrarajan, M. (2014). A Green Approach for the Synthesis of a Cerium Oxide Nanoparticle: Characterization and Antibacterial Activity. Int. J. Nanosci. 13, 1450018. doi:10.1142/S0219581X14500185

Kanwal, Z., Raza, M., Manzoor, F., Riaz, S., Jabeen, G., Fatima, S., et al. (2019). A Comparative Assessment of Nanotoxicity Induced by Metal (Silver, Nickel) and Metal Oxide (Cobalt, Chromium) Nanoparticles in Labeo Rohita. Nanomaterials 9, 309. doi:10.3390/nano9020309

Karade, V. C., Parit, S. B., Dawkar, V. V., Devan, R. S., Choudhary, R. J., Kedge, V. V., et al. (2019). A Green Approach for the Synthesis of $\alpha$-Fe2O3 Nanoparticles from Gardenia Resinifera Plant and It's In Vitro Hyperthermia Application. Heliyon 5, e02044. doi:10.1016/j.heliyon.2019.e02044

Karthik, C., and Radha, K. V. (2012). Biosynthesis and Characterization of Silver Nanoparticles Using enterobacter Aerogenes: A Kinetic Approach. Dig. J. Nanomater. Biostructures 7, 1007-1014.

Kathiraven, T., Sundaramanickam, A., Shanmugam, N., and Balasubramanian, T. (2015). Green Synthesis of Silver Nanoparticles Using marine Algae Caulerpa Racemosa and Their Antibacterial Activity Against Some Human Pathogens. Appl. Nanosci. 5, 499-504. doi:10.1007/s13204-014-0341-2 
Khatami, M., Pourseyedi, S., Khatami, M., Hamidi, H., Zaeifi, M., and Soltani, L. (2015). Synthesis of Silver Nanoparticles Using Seed Exudates of Sinapis Arvensis as a Novel Bioresource, and Evaluation of Their Antifungal Activity. Bioresour. Bioproc. 2, 19. doi:10.1186/s40643-015-0043-y

Khatoon, Z., McTiernan, C. D., Suuronen, E. J., Mah, T.-F., and Alarcon, E. I. (2018). Bacterial Biofilm Formation on Implantable Devices and Approaches to its Treatment and Prevention. Heliyon 4, e01067. doi:10.1016/ j.heliyon.2018.e01067

Khodashenas, B., and Ghorbani, H. R. (2014). Synthesis of Copper Nanoparticles : An Overview of the Various Methods. Korean J. Chem. Eng. 31, 1105-1109. doi:10.1007/s11814-014-0127-y

Kimber, R. L., Lewis, E. A., Parmeggiani, F., Smith, K., Bagshaw, H., Starborg, T., et al. (2018). Biosynthesis and Characterization of Copper Nanoparticles UsingShewanella Oneidensis: Application for Click Chemistry. Small 14, 1703145. doi:10.1002/smll.201703145

Kirmusaoglu, S. (2016). Staphylococcal Biofilms: Pathogenicity, Mechanism and Regulation of Biofilm Formation by Quorum-Sensing System and Antibiotic Resistance Mechanisms of Biofilm-Embedded Microorganisms. In Microbial Biofilms - Importance and Applications. doi:10.5772/62943

Kokare, C. R., Chakraborty, S., Khopade, A. N., and Mahadik, K. R. (2009). Biofilm: Importance and Applications. Indian J. Biotechnol. 8, 159-168.

Kora, A. J., Beedu, S. R., and Jayaraman, A. (2012). Size-controlled green Synthesis of Silver Nanoparticles Mediated by Gum Ghatti (Anogeissus Latifolia) and its Biological Activity. Org. Med. Chem. Lett. 2, 17. doi:10.1186/2191-2858-2-17

Korbekandi, H., Mohseni, S., Mardani Jouneghani, R., Pourhossein, M., and Iravani, S. (2016). Biosynthesis of Silver Nanoparticles usingSaccharomyces Cerevisiae. Artif. Cell Nanomedicine, Biotechnol. 44, 235-239. doi:10.3109/ 21691401.2014.937870

Korkmaz, N., Ceylan, Y., Hamid, A., Karadağ, A., Bülbül, A. S., Aftab, M. N., et al. (2020). Biogenic Silver Nanoparticles Synthesized via Mimusops Elengi Fruit Extract, a Study on Antibiofilm, Antibacterial, and Anticancer Activities. J. Drug Deliv. Sci. Technology 59, 101864. doi:10.1016/j.jddst.2020.101864

Kuhn, D. M., George, T., Chandra, J., Mukherjee, P. K., and Ghannoum, M. A. (2002). Antifungal Susceptibility of Candida Biofilms: Unique Efficacy of Amphotericin B Lipid Formulations and Echinocandins. Antimicrob. Agents Chemother. 46, 1773-1780. doi:10.1128/AAC.46.6.1773-1780.2002

Kulshrestha, S., Qayyum, S., and Khan, A. U. (2017). Antibiofilm Efficacy of green Synthesized Graphene Oxide-Silver Nanocomposite Using Lagerstroemia Speciosa floral Extract: A Comparative Study on Inhibition of GramPositive and Gram-Negative Biofilms. Microb. Pathogenesis 103, 167-177. doi:10.1016/j.micpath.2016.12.022

Kumar, P., Selvi, S. S., Prabha, A. L., Kumar, K. P., Ganeshkumar, R. S., and Govindaraju, M. (2012). Synthesis of Silver Nanoparticles from Sargassum Tenerrimum and Screening Phytochemicals for its Antibacterial Activity. Nano Biomed. Eng. 4, 12-16. doi:10.5101/nbe.v4i1.p12-16

Kumar, S., Lather, V., and Pandita, D. (2015). Green Synthesis of Therapeutic Nanoparticles: An Expanding Horizon. Nanomedicine 10, 2451-2471. doi:10.2217/nnm.15.112

Kuppusamy, P., Yusoff, M. M., Ichwan, S. J. A., Parine, N. R., Maniam, G. P., and Govindan, N. (2015). Commelina Nudiflora L. Edible weed as a Novel Source for Gold Nanoparticles Synthesis and Studies on Different Physical-Chemical and Biological Properties. J. Ind. Eng. Chem. 27, 59-67. doi:10.1016/ j.jiec.2014.11.045

Kuppusamy, P., Yusoff, M. M., Maniam, G. P., and Govindan, N. (2016). Biosynthesis of Metallic Nanoparticles Using Plant Derivatives and Their New Avenues in Pharmacological Applications - An Updated Report. Saudi Pharm. J. 24, 473-484. doi:10.1016/j.jsps.2014.11.013

Küünal, S., Rauwel, P., and Rauwel, E. (2018). Plant Extract Mediated Synthesis of Nanoparticles. In Emerging Applications of Nanoparticles and Architectural Nanostructures: Current Prospects and Future Trends, 411-446. doi:10.1016/ B978-0-323-51254-1.00014-2

Lahiri, D., Nag, M., Sheikh, H. I., Sarkar, T., Edinur, H. A., Pati, S., et al. (2021). Microbiologically-Synthesized Nanoparticles and Their Role in Silencing the Biofilm Signaling Cascade. Front. Microbiol. 12, 636588. doi:10.3389/ fmicb.2021.636588

Landini, P., Antoniani, D., Burgess, J. G., and Nijland, R. (2010). Molecular Mechanisms of Compounds Affecting Bacterial Biofilm Formation and Dispersal. Appl. Microbiol. Biotechnol. 86, 813-823. doi:10.1007/s00253-010-2468-8
Lanone, S., and Boczkowski, J. (2006). Biomedical Applications and Potential Health Risks of Nanomaterials: Molecular Mechanisms. Cmm 6, 651-663. doi: $10.2174 / 156652406778195026$

Lateef, A., Ojo, S. A., and Elegbede, J. A. (2016). The Emerging Roles of Arthropods and Their Metabolites in the Green Synthesis of Metallic Nanoparticles. Nanotechnol. Rev. 5, 601-622. doi:10.1515/ntrev-2016-0049

Lebeaux, D., Fernández-Hidalgo, N., Chauhan, A., Lee, S., Ghigo, J. M., Almirante, B., et al. (2014). Management of Infections Related to Totally Implantable Venous-Access Ports: Challenges and Perspectives. Lancet Infect. Dis. 14, 146-159. doi:10.1016/S1473-3099(13)70266-4

Lemfack, M.-C., Bahl, H., Piechulla, B., and Magnus, N. (2020). "The Domain of Bacteria and Their Volatile Metabolic Potential," in Bacterial Volatile Compounds as Mediators of Airborne Interactions, 1-38. doi:10.1007/978981-15-7293-7_1

Lesniak, A., Salvati, A., Santos-Martinez, M. J., Radomski, M. W., Dawson, K. A., and Åberg, C. (2013). Nanoparticle Adhesion to the Cell Membrane and its Effect on Nanoparticle Uptake Efficiency. J. Am. Chem. Soc. 135, 1438-1444. doi:10.1021/ja309812z

Lewis, K. (2001). Riddle of Biofilm Resistance. Antimicrob. Agents Chemother. 45 , 999-1007. doi:10.1128/AAC.45.4.999-1007.2001

Li, X., Xu, H., Chen, Z.-S., and Chen, G. (2011). Biosynthesis of Nanoparticles by Microorganisms and Their Applications. J. Nanomater. 2011, 1-16. doi:10.1155/2011/270974

Lombardi, A. T., and Garcia, O. (1999). An Evaluation into the Potential of Biological Processing for the Removal of Metals from Sewage Sludges. Crit. Rev. Microbiol. 25, 275-288. doi:10.1080/10408419991299239

Luo, Y.-H., Chang, L. W., and Lin, P. (2015). Metal-Based Nanoparticles and the Immune System: Activation, Inflammation, and Potential Applications. Biomed. Res. Int. 2015, 1-12. doi:10.1155/2015/143720

Macfarlane, S., and Dillon, J. F. (2007). Microbial Biofilms in the Human Gastrointestinal Tract. J. Appl. Microbiol. 102, 1187-1196. doi:10.1111/ j.1365-2672.2007.03287.x

Mahmoodi, S., Elmi, A., and Hallaj Nezhadi, S. (2018). Copper Nanoparticles as Antibacterial Agents. J. Mol. Pharm. Org. Process. Res. 6, 140. doi:10.4172/23299053.1000140

Majumdar, M., Khan, S. A., Biswas, S. C., Roy, D. N., Panja, A. S., and Misra, T. K. (2020). In Vitro and In Silico Investigation of Anti-biofilm Activity of Citrus Macroptera Fruit Extract Mediated Silver Nanoparticles. J. Mol. Liquids 302, 112586. doi:10.1016/j.molliq.2020.112586

Makarov, V. V., Love, A. J., Sinitsyna, O. V., Makarova, S. S., Yaminsky, I. V., Taliansky, M. E., et al. (2014). “Green” Nanotechnologies: Synthesis of Metal Nanoparticles Using Plants. Acta Naturae 6, 35-44. doi:10.32607/207582512014-6-1-35-44

Malaikozhundan, B., Vaseeharan, B., Vijayakumar, S., Sudhakaran, R., Gobi, N., and Shanthini, G. (2016). Antibacterial and Antibiofilm Assessment of Momordica Charantia Fruit Extract Coated Silver Nanoparticle. Biocatal. Agric. Biotechnol. 8, 189-196. doi:10.1016/j.bcab.2016.09.007

Malaikozhundan, B., Vinodhini, J., Kalanjiam, M. A. R., Vinotha, V., Palanisamy, S., Vijayakumar, S., et al. (2020). High Synergistic Antibacterial, Antibiofilm, Antidiabetic and Antimetabolic Activity of Withania Somnifera Leaf ExtractAssisted Zinc Oxide Nanoparticle. Bioproc. Biosyst. Eng. 43, 1533-1547. doi:10.1007/s00449-020-02346-0

Malhotra, V., Chandra, P., and Maurya, P. K. (2015). Control of Bacterial Biofilms in Industrial and Medical Settings. 6, 1-4.

Mandal, D., Bolander, M. E., Mukhopadhyay, D., Sarkar, G., and Mukherjee, P. (2006). The Use of Microorganisms for the Formation of Metal Nanoparticles and Their Application. Appl. Microbiol. Biotechnol. 69, 485-492. doi:10.1007/ s00253-005-0179-3

Manik, U. P., Nande, A., Raut, S., and Dhoble, S. J. (2020). Green Synthesis of Silver Nanoparticles Using Plant Leaf Extraction of Artocarpus Heterophylus and Azadirachta indica. Results Mater. 6, 100086. doi:10.1016/ j.rinma.2020.100086

Manju, S., Malaikozhundan, B., Vijayakumar, S., Shanthi, S., Jaishabanu, A., Ekambaram, P., et al. (2016). Antibacterial, Antibiofilm and Cytotoxic Effects of Nigella Sativa Essential Oil Coated Gold Nanoparticles. Microb. Pathogenesis 91, 129-135. doi:10.1016/j.micpath.2015.11.021

Mata, R., Nakkala, J. R., and Sadras, S. R. (2015). Biogenic Silver Nanoparticles from Abutilon Indicum: Their Antioxidant, Antibacterial and Cytotoxic Effects 
In Vitro. Colloids Surf. B: Biointerfaces 128, 276-286. doi:10.1016/ j.colsurfb.2015.01.052

Miglani, S., and Tani-Ishii, N. (2021). Biosynthesized Selenium Nanoparticles: Characterization, Antimicrobial, and Antibiofilm Activity Against Enterococcus faecalis. PeerJ 9, e11653. doi:10.7717/peerj.11653

Mirzaei, H., and Darroudi, M. (2017). Zinc Oxide Nanoparticles: Biological Synthesis and Biomedical Applications. Ceramics Int. 43, 907-914. doi:10.1016/j.ceramint.2016.10.051

Mittal, A. K., Chisti, Y., and Banerjee, U. C. (2013). Synthesis of Metallic Nanoparticles Using Plant Extracts. Biotechnol. Adv. 31, 346-356. doi:10.1016/j.biotechadv.2013.01.003

Mohammad Reza, S. (2018). Bacterial Biofilm and its Clinical Implications. Ann. Microbiol. Res. 2, 45-50. doi:10.36959/958/568

Mohammed, A. B. A., Hegazy, A. E., and Salah, A. (2021). Novelty of Synergistic and Cytotoxicity Activities of Silver Nanoparticles Produced by Lactobacillus Acidophilus. Appl. Nanosci., 1-8. doi:10.1007/s13204-021-01878-5

Mohanta, Y. K., Biswas, K., Jena, S. K., Hashem, A., Abd_Allah, E. F., and Mohanta, T. K. (2020). Anti-biofilm and Antibacterial Activities of Silver Nanoparticles Synthesized by the Reducing Activity of Phytoconstituents Present in the Indian Medicinal Plants. Front. Microbiol. 11, 1143. doi:10.3389/ fmicb.2020.01143

Moodley, J. S., Naidu Krishna, S. B., Pillay, K., and Govender, P. (2018). Production, Characterization and Antimicrobial Activity of Silver Nanoparticles Produced by Pediococcus Acidilactici. Dig. J. Nanomater. Biostructures 13, 77-86.

Mortimer, M., Kasemets, K., Vodovnik, M., Marinšek-Logar, R., and Kahru, A. (2011). Exposure to CuO Nanoparticles Changes the Fatty Acid Composition of Protozoa tetrahymena Thermophila. Environ. Sci. Technol. 45, 6617-6624. doi:10.1021/es201524q

Moulavi, P., Noorbazargan, H., Dolatabadi, A., Foroohimanjili, F., Tavakoli, Z., Mirzazadeh, S., et al. (2019). Antibiofilm Effect of green Engineered Silver Nanoparticles Fabricated fromArtemisia Scoporiaextract on the Expression oficaAandicaRgenes against Multidrug-resistantStaphylococcus Aureus. J. Basic Microbiol. 59, 701-712. doi:10.1002/jobm.201900096

Mrsic, Z., Hopkins, S. P., Antevil, J. L., and Mullenix, P. S. (2018). Valvular Heart Disease. Prim. Care Clin. Off. Pract. 45, 81-94. doi:10.1016/j.pop.2017.10.002

Muhammad, M. H., Idris, A. L., Fan, X., Guo, Y., Yu, Y., Jin, X., et al. (2020). Beyond Risk: Bacterial Biofilms and Their Regulating Approaches. Front. Microbiol. 11, 928. doi:10.3389/fmicb.2020.00928

Mukherjee, P., Ahmad, A., Mandal, D., Senapati, S., Sainkar, S. R., Khan, M. I., et al. (2001). Fungus-Mediated Synthesis of Silver Nanoparticles and Their Immobilization in the Mycelial Matrix: A Novel Biological Approach to Nanoparticle Synthesis. Nano Lett. 1, 515-519. doi:10.1021/nl0155274

Muthamil, S., Devi, V. A., Balasubramaniam, B., Balamurugan, K., and Pandian, S. K. (2018). Green Synthesized Silver Nanoparticles Demonstrating Enhanced In Vitro and In Vivo Antibiofilm Activity Against Candida Spp. J. Basic Microbiol. 58, 343-357. doi:10.1002/jobm.201700529

Muthuraman, M. S., Nithya, S., Vinoth kumar, V., Christena, L. R., Vadivel, V., Subramanian, N. S., et al. (2019). Green Synthesis of Silver Nanoparticles Using Nardostachys Jatamansi and Evaluation of its Anti-biofilm Effect against Classical Colonizers. Microb. Pathogenesis 126, 1-5. doi:10.1016/ j.micpath.2018.10.024

Nallasamy, P., M, V., Phuong, N.-T., Kathirvel, B., and A, P. (2021). Enhanced Antimicrobial, Antibiofilm and Anticancer Activities of Biocompatible Neem Gum Coated Palladium Nanoparticles. Prog. Org. Coat. 151, 106098. doi:10.1016/j.porgcoat.2020.106098

Nadell, C. D., Xavier, J. B., and Foster, K. R. (2009). The Sociobiology of Biofilms. FEMS Microbiol. Rev. 33, 206-224. doi:10.1111/j.1574-6976.2008.00150.x

Naidi, S. N., Khan, F., Tan, A. L., Harunsani, M. H., Kim, Y.-M., and Khan, M. M. (2021a). Green Synthesis of CeO2 and Zr/Sn-Dual Doped CeO2 Nanoparticles with Photoantioxidant and Antibiofilm Activities. Biomater. Sci. 9, 4854-4869. doi:10.1039/d1bm00298h

Naidi, S. N., Khan, F., Tan, A. L., Harunsani, M. H., Kim, Y.-M., and Khan, M. M. (2021b). Photoantioxidant and Antibiofilm Studies of green Synthesized SnDoped CeO2 Nanoparticles Using Aqueous Leaf Extracts of Pometia Pinnata. New J. Chem. 45, 7816-7829. doi:10.1039/d1nj00416f
Nam, N. H., and Luong, N. H. (2019). Nanoparticles: Synthesis and Applications. Materials for Biomedical Engineering 211-240. doi:10.1016/B978-0-08-1028148.00008-1

Namasivayam, S. K. R., and Chitrakala, K. (2011). Ecotoxicological Effect of Lecanicillium Lecanii (Ascomycota: Hypocreales) Based Silver Nanoparticles on Growth Parameters of Economically Important Plants. J. Biopestic. 4, 97-101.

Narayanan, M., Devi, P. G., Natarajan, D., Kandasamy, S., Devarayan, K., Alsehli, M., et al. (2021). Green Synthesis and Characterization of Titanium Dioxide Nanoparticles Using Leaf Extract of Pouteria Campechiana and Larvicidal and Pupicidal Activity on Aedes aegypti. Environ. Res. 200, 111333. doi:10.1016/ j.envres.2021.111333

Naseem, T., and Farrukh, M. A. (2015). Antibacterial Activity of Green Synthesis of Iron Nanoparticles UsingLawsonia inermisandGardenia jasminoidesLeaves Extract. J. Chem. 2015, 1-7. doi:10.1155/2015/912342

Naseer, M., Ramadan, R., Xing, J., and Samak, N. A. (2021). Facile green Synthesis of Copper Oxide Nanoparticles for the Eradication of Multidrug Resistant Klebsiella Pneumonia and Helicobacter pylori Biofilms. Int. Biodeterioration Biodegradation 159, 105201. doi:10.1016/j.ibiod.2021.105201

Nath, D., and Banerjee, P. (2013). Green Nanotechnology - A new hope for Medical Biology. Environ. Toxicol. Pharmacol. 36, 997-1014. doi:10.1016/ j.etap.2013.09.002

Neethirajan, S., Clond, M. A., and Vogt, A. (2014). Medical BiofilmsNanotechnology Approaches. J. Biomed. Nanotechnol. 10, 2806-2827. doi:10.1166/jbn.2014.1892

Neihaya, H. Z., and Zaman, H. H. (2018). Investigating the Effect of Biosynthesized Silver Nanoparticles as Antibiofilm on Bacterial Clinical Isolates. Microb. Pathogenesis 116, 200-208. doi:10.1016/j.micpath.2018.01.024

Nel, A., Xia, T., Mädler, L., and Li, N. (2006). Toxic Potential of Materials at the Nanolevel. Science 311, 622-627. doi:10.1126/science.1114397

Nesrin, K., Yusuf, C., Ahmet, K., Ali, S. B., Muhammad, N. A., Suna, S., et al. (2020). Biogenic Silver Nanoparticles Synthesized from Rhododendron Ponticum and Their Antibacterial, Antibiofilm and Cytotoxic Activities. J. Pharm. Biomed. Anal. 179, 112993. doi:10.1016/j.jpba.2019.112993

Noruzi, M. (2015). Biosynthesis of Gold Nanoparticles Using Plant Extracts. Bioproc. Biosyst. Eng. 38, 1-14. doi:10.1007/s00449-014-1251-0

Obeizi, Z., Benbouzid, H., Ouchenane, S., Yılmaz, D., Culha, M., and Bououdina, M. (2020). Biosynthesis of Zinc Oxide Nanoparticles from Essential Oil of Eucalyptus Globulus with Antimicrobial and Anti-biofilm Activities. Mater. Today Commun. 25, 101553. doi:10.1016/j.mtcomm.2020.101553

Oshiro, K. G. N., Rodrigues, G., Monges, B. E. D., Cardoso, M. H., and Franco, O. L. (2019). Bioactive Peptides against Fungal Biofilms. Front. Microbiol. 10. doi:10.3389/fmicb.2019.02169

Otari, S. V., Patil, R. M., Nadaf, N. H., Ghosh, S. J., and Pawar, S. H. (2012). Green Biosynthesis of Silver Nanoparticles from an Actinobacteria Rhodococcus Sp. Mater. Lett. 72, 92-94. doi:10.1016/j.matlet.2011.12.109

Ovais, M., Raza, A., Naz, S., Islam, N. U., Khalil, A. T., Ali, S., et al. (2017). Current State and Prospects of the Phytosynthesized Colloidal Gold Nanoparticles and Their Applications in Cancer Theranostics. Appl. Microbiol. Biotechnol. 101, 3551-3565. doi:10.1007/s00253-017-8250-4

Overman, P. R. (2000). Biofilm: A New View of Plaque. J. Contemp. Dent. Pract. 1, 37-44. doi:10.5005/jcdp-1-3-37

Pajkos, A., Deva, A. K., Vickery, K., Cope, C., Chang, L., and Cossart, Y. E. (2003) Detection of Subclinical Infection in Significant Breast Implant Capsules. Plast. Reconstr. Surg. 111, 1605-1611. doi:10.1097/01.PRS.0000054768.14922.44

Pal, G., Rai, P., and Pandey, A. (2019). "Green Synthesis of Nanoparticles: A Greener Approach for a Cleaner Future," in Green Synthesis, Characterization and Applications of Nanoparticles, 1-26. doi:10.1016/b978-0-08-1025796.00001-0

Paluch, E., Rewak-Soroczyńska, J., Jędrusik, I., Mazurkiewicz, E., and Jermakow, K. (2020). Prevention of Biofilm Formation by Quorum Quenching. Appl. Microbiol. Biotechnol. 104, 1871-1881. doi:10.1007/s00253-020-10349-w

Patil, M. P., and Kim, G.-D. (2017). Eco-friendly Approach for Nanoparticles Synthesis and Mechanism behind Antibacterial Activity of Silver and Anticancer Activity of Gold Nanoparticles. Appl. Microbiol. Biotechnol. 101, 79-92. doi:10.1007/s00253-016-8012-8 
Patra, J. K., and Baek, K.-H. (2014). Green Nanobiotechnology: Factors Affecting Synthesis and Characterization Techniques. J. Nanomater. 2014, 1-12. doi:10.1155/2014/417305

Paulkumar, K., Gnanajobitha, G., Vanaja, M., Rajeshkumar, S., Malarkodi, C., Pandian, K., et al. (2014). Piper nigrumLeaf and Stem Assisted Green Synthesis of Silver Nanoparticles and Evaluation of its Antibacterial Activity against Agricultural Plant Pathogens. Scientific World J. 2014, 1-9. doi:10.1155/2014/ 829894

Pelling, H., Nzakizwanayo, J., Milo, S., Denham, E. L., MacFarlane, W. M., Bock, L. J., et al. (2019). Bacterial Biofilm Formation on Indwelling Urethral Catheters. Lett. Appl. Microbiol. 68, 277-293. doi:10.1111/lam.13144

Percival, S. L., Hill, K. E., Williams, D. W., Hooper, S. J., Thomas, D. W., and Costerton, J. W. (2012). A Review of the Scientific Evidence for Biofilms in Wounds. Wound Repair Regen. 20, 647-657. doi:10.1111/j.1524475X.2012.00836.X

Perveen, K., Husain, F. M., Qais, F. A., Khan, A., Razak, S., Afsar, T., et al. (2021). Microwave-assisted Rapid green Synthesis of Gold Nanoparticles Using Seed Extract of Trachyspermum Ammi: Ros Mediated Biofilm Inhibition and Anticancer Activity. Biomolecules 11, 197. doi:10.3390/biom11020197

Pinto, R. M., Soares, F. A., Reis, S., Nunes, C., and Van Dijck, P. (2020). Innovative Strategies toward the Disassembly of the EPS Matrix in Bacterial Biofilms. Front. Microbiol. 11, 952. doi:10.3389/fmicb.2020.00952

Ponde, N. O., Lortal, L., Ramage, G., Naglik, J. R., and Richardson, J. P. (2021). Candida Albicans Biofilms and Polymicrobial Interactions. Crit. Rev. Microbiol. 47, 91-111. doi:10.1080/1040841X.2020.1843400

Prameela Devi, T., Kulanthaivel, S., Kamil, D., Borah, J. L., Prabhakaran, N., and Srinivasa, N. (2013). Biosynthesis of Silver Nanoparticles from Trichoderma Species. Indian J. Exp. Biol. 51, 543-547. doi:10.1016/j.colsurfb.2009.08.028

Prasad, A., Baker, S., Nagendra Prasad, M. N., Devi, A. T., Satish, S., Zameer, F., et al. (2019). Phytogenic Synthesis of Silver Nanobactericides for Anti-biofilm Activity Against Human Pathogen H. pylori. SN Appl. Sci. 1. doi:10.1007/ s42452-019-0362-2

Priyadarshini, E., Priyadarshini, S. S., and Pradhan, N. (2019). Heavy Metal Resistance in Algae and its Application for Metal Nanoparticle Synthesis. Appl. Microbiol. Biotechnol. 103, 3297-3316. doi:10.1007/s00253-019-09685-3

Punniyakotti, P., Panneerselvam, P., Perumal, D., Aruliah, R., and Angaiah, S. (2020). Anti-bacterial and Anti-biofilm Properties of green Synthesized Copper Nanoparticles from Cardiospermum Halicacabum Leaf Extract. Bioproc. Biosyst. Eng. 43, 1649-1657. doi:10.1007/s00449-020-02357-x

Qais, F. A., Ahmad, I., Altaf, M., and Alotaibi, S. H. (2021). Biofabrication of Gold Nanoparticles Using Capsicum Annuum Extract and its Antiquorum Sensing and Antibiofilm Activity Against Bacterial Pathogens. ACS Omega 6, 16670-16682. doi:10.1021/acsomega.1c02297

Qais, F. A., Shafiq, A., Ahmad, I., Husain, F. M., Khan, R. A., and Hassan, I. (2020). Green Synthesis of Silver Nanoparticles Using Carum Copticum: Assessment of its Quorum Sensing and Biofilm Inhibitory Potential against Gram Negative Bacterial Pathogens. Microb. Pathogenesis 144, 104172. doi:10.1016/ j.micpath.2020.104172

Qayyum, S., and Khan, A. U. (2016). Nanoparticles vs. Biofilms: a Battle against Another Paradigm of Antibiotic Resistance. Med. Chem. Commun. 7, 1479-1498. doi:10.1039/c6md00124f

Raad, I., Costerton, W., Sabharwal, U., Sadlowski, M., Anaissie, E., and Bodey, G. P. (1993). Ultrastructural Analysis of Indwelling Vascular Catheters: A Quantitative Relationship between Luminal Colonization and Duration of Placement. J. Infect. Dis. 168, 400-407. doi:10.1093/infdis/168.2.400

Rada, B. (2017). Interactions between Neutrophils and pseudomonas Aeruginosa in Cystic Fibrosis. Pathogens 6, 10. doi:10.3390/pathogens6010010

Radu, M., Munteanu, M. C., Petrache, S., Serban, A. I., Dinu, D., Hermenean, A., et al. (2010). Depletion of Intracellular Glutathione and Increased Lipid Peroxidation Mediate Cytotoxicity of Hematite Nanoparticles in MRC-5 Cells. Acta Biochim. Pol. 57, 355-360. doi:10.18388/abp.2010_2416

Raheman, F., Deshmukh, S., Ingle, A., Gade, A., and Rai, M. (2011). Silver Nanoparticles: Novel Antimicrobial Agent Synthesized from an Endophytic Fungus Pestalotia Sp. Isolated from Leaves of Syzygium Cumini (L). Nano Biomed. Eng. 3, 174-178. doi:10.5101/nbe.v3i3.p174-178

Rahimi, G., Alizadeh, F., and Khodavandi, A. (2016). Mycosynthesis of Silver Nanoparticles from Candida Albicans and its Antibacterial Activity against
Escherichia coli and Staphylococcus aureus. Trop. J. Pharm. Res. 15, 371. doi:10.4314/tjpr.v15i2.21

Rajeshkumar, S., Malarkodi, C., Gnanajobitha, G., Paulkumar, K., Vanaja, M., Kannan, C., et al. (2013). Seaweed-mediated Synthesis of Gold Nanoparticles Using Turbinaria Conoides and its Characterization. J. Nanostruct Chem. 3, 1-7. doi:10.1186/2193-8865-3-44

Rajkumari, J., Magdalane, C. M., Siddhardha, B., Madhavan, J., Ramalingam, G., Al-Dhabi, N. A., et al. (2019). Synthesis of Titanium Oxide Nanoparticles Using Aloe Barbadensis Mill and Evaluation of its Antibiofilm Potential against Pseudomonas aeruginosa PAO1. J. Photochem. Photobiol. B: Biol. 201, 111667. doi:10.1016/j.jphotobiol.2019.111667

Rajput, N., and Bankar, A. (2017). Bio-inspired Gold Nanoparticles Synthesis and Their Anti-biofilm Efficacy. J. Pharm. Invest. 47, 521-530. doi:10.1007/s40005016-0280-x

Ramage, G., and Williams, C. (2013). The Clinical Importance of Fungal Biofilms. Adv. Appl. Microbiol., 27-83. doi:10.1016/B978-0-12-407673-0.00002-3

Ramalingam, V., Dhinesh, P., Sundaramahalingam, S., and Rajaram, R. (2019). Green Fabrication of Iron Oxide Nanoparticles Using Grey Mangrove Avicennia marina for Antibiofilm Activity and In Vitro Toxicity. Surf. Inter. 15, 70-77. doi:10.1016/j.surfin.2019.01.008

Ramasamy, M., and Lee, J. (2016). Recent Nanotechnology Approaches for Prevention and Treatment of Biofilm-Associated Infections on Medical Devices. Biomed. Res. Int. 2016, 1-17. doi:10.1155/2016/1851242

Ramezani, F., Jebali, A., and Kazemi, B. (2012). A Green Approach for Synthesis of Gold and Silver Nanoparticles by Leishmania Sp. Appl. Biochem. Biotechnol. 168, 1549-1555. doi:10.1007/s12010-012-9877-3

Ravindran, D., Ramanathan, S., Arunachalam, K., Jeyaraj, G. P., Shunmugiah, K. P., and Arumugam, V. R. (2018). Phytosynthesized Silver Nanoparticles as Antiquorum Sensing and Antibiofilm Agent against the Nosocomial Pathogen Serratia marcescens : an In Vitro Study. J. Appl. Microbiol. 124, 1425-1440. doi:10.1111/jam.13728

Renner, L. D., and Weibel, D. B. (2011). Physicochemical Regulation of Biofilm Formation. MRS Bull. 36, 347-355. doi:10.1557/mrs.2011.65

Roopan, S. M., RohitMadhumitha, G., Madhumitha, A. A., Kamaraj, C., Bharathi, A., and Surendra, T. V. (2013). Low-cost and Eco-Friendly Phyto-Synthesis of Silver Nanoparticles Using Cocos Nucifera Coir Extract and its Larvicidal Activity. Ind. Crops Prod. 43, 631-635. doi:10.1016/j.indcrop.2012.08.013

Rumbaugh, K. P., and Sauer, K. (2020). Biofilm Dispersion. Nat. Rev. Microbiol. 18, 571-586. doi:10.1038/s41579-020-0385-0

Sadeghi, B., Rostami, A., and Momeni, S. S. (2015). Facile green Synthesis of Silver Nanoparticles Using Seed Aqueous Extract of Pistacia Atlantica and its Antibacterial Activity. Spectrochimica Acta Part A: Mol. Biomol. Spectrosc. 134, 326-332. doi:10.1016/j.saa.2014.05.078

Saleem, S., Ahmed, B., Khan, M. S., Al-Shaeri, M., and Musarrat, J. (2017). Inhibition of Growth and Biofilm Formation of Clinical Bacterial Isolates by NiO Nanoparticles Synthesized from Eucalyptus Globulus Plants. Microb. Pathogenesis 111, 375-387. doi:10.1016/j.micpath.2017.09.019

Santos, C. M. A., Pires, M. C. V., Leão, T. L., Hernández, Z. P., Rodriguez, M. L., Martins, A. K. S., et al. (2016). Selection of Lactobacillus Strains as Potential Probiotics for Vaginitis Treatment. Microbiol. (United Kingdom) 162, 1195-1207. doi:10.1099/mic.0.000302

Sarathi Kannan, D., Mahboob, S., Al-Ghanim, K. A., and Venkatachalam, P. (2021). Antibacterial, Antibiofilm and Photocatalytic Activities of Biogenic Silver Nanoparticles from Ludwigia Octovalvis. J. Clust. Sci. 32, 255-264. doi:10.1007/s10876-020-01784-w

Saravanan, M., Vemu, A. K., and Barik, S. K. (2011). Rapid Biosynthesis of Silver Nanoparticles from bacillus Megaterium (Ncim 2326) and Their Antibacterial Activity on Multi Drug Resistant Clinical Pathogens. Colloids Surf. B: Biointerfaces 88, 325-331. doi:10.1016/j.colsurfb.2011.07.009

Sathishkumar, Y., Devarayan, K., Ki, C., Rajagopal, K., and Soo Lee, Y. (2015). Shape-controlled Extracellular Synthesis of Silver Nanocubes by Mucor Circinelloides. Mater. Lett. 159, 481-483. doi:10.1016/j.matlet.2015.06.124

Sau, T. K., and Rogach, A. L. (2010). Nonspherical noble Metal Nanoparticles: Colloid-Chemical Synthesis and Morphology Control. Adv. Mater. 22, 1781-1804. doi:10.1002/adma.200901271

Schieber, M., and Chandel, N. S. (2014). ROS Function in Redox Signaling and Oxidative Stress. Curr. Biol. 24, R453-R462. doi:10.1016/j.cub.2014.03.034 
Schilder, A. G. M., Marom, T., Bhutta, M. F., Casselbrant, M. L., Coates, H., Gisselsson-Solén, M., et al. (2017). Panel 7: Otitis Media: Treatment and Complications. Otolaryngol. Head Neck Surg. 156, S88-S105. doi:10.1177/ 0194599816633697

Schrand, A. M., Rahman, M. F., Hussain, S. M., Schlager, J. J., Smith, D. A., and Syed, A. F. (2010). Metal-based Nanoparticles and Their Toxicity Assessment. WIREs Nanomed Nanobiotechnol 2, 544-568. doi:10.1002/wnan.103

Seshadri, S., Saranya, K., and Kowshik, M. (2011). Green Synthesis of lead Sulfide Nanoparticles by the lead Resistant marine Yeast, Rhodosporidium Diobovatum. Biotechnol. Prog. 27, 1464-1469. doi:10.1002/btpr.651

Shafiq, S. A., H, A. R., and Majeed, H. Z. (2016). Study of Biosynthesis Silver Nanoparticles by Fusarium Graminaerum and Test Their Antimicrobial Activity. Int. J. Innov. Appl. Stud. 15, 43-50.

Shah, M., Fawcett, D., Sharma, S., Tripathy, S., and Poinern, G. (2015). Green Synthesis of Metallic Nanoparticles via Biological Entities. Materials 8, 7278-7308. doi:10.3390/ma8115377

Shah, S., Gaikwad, S., Nagar, S., Kulshrestha, S., Vaidya, V., Nawani, N., et al. (2019). Biofilm Inhibition and Anti-Quorum Sensing Activity of Phytosynthesized Silver Nanoparticles against the Nosocomial Pathogen Pseudomonas aeruginosa. Biofouling 35, 34-49. doi:10.1080/ 08927014.2018 .1563686

Shakibaie, M., Forootanfar, H., Golkari, Y., Mohammadi-Khorsand, T., and Shakibaie, M. R. (2015). Anti-biofilm Activity of Biogenic Selenium Nanoparticles and Selenium Dioxide against Clinical Isolates of Staphylococcus aureus, Pseudomonas aeruginosa, and Proteus mirabilis. J. Trace Elem. Med. Biol. 29, 235-241. doi:10.1016/j.jtemb.2014.07.020

Shankar, S. S., Rai, A., Ahmad, A., and Sastry, M. (2004). Rapid Synthesis of Au, Ag, and Bimetallic Au Core-Ag Shell Nanoparticles Using Neem (Azadirachta indica) Leaf Broth. J. Colloid Interf. Sci. 275, 496-502. doi:10.1016/j.jcis.2004.03.003

Sharahi, J. Y., Azimi, T., Shariati, A., Safari, H., Tehrani, M. K., and Hashemi, A. (2019). Advanced Strategies for Combating Bacterial Biofilms. J. Cel. Physiol. 234, 14689-14708. doi:10.1002/jcp.28225

Sharma, D., Kanchi, S., and Bisetty, K. (2019). Biogenic Synthesis of Nanoparticles: A Review. Arabian J. Chem. 12, 3576-3600. doi:10.1016/j.arabjc.2015.11.002

Shkodenko, L., Kassirov, I., and Koshel, E. (2020). Metal Oxide Nanoparticles against Bacterial Biofilms: Perspectives and Limitations. Microorganisms 8, 1545. doi:10.3390/microorganisms 8101545

Simeonidis, K., Mourdikoudis, S., Moulla, M., Tsiaoussis, I., Martinez-Boubeta, C., Angelakeris, M., et al. (2007). Controlled Synthesis and Phase Characterization of Fe-Based Nanoparticles Obtained by thermal Decomposition. J. Magnetism Magn. Mater. 316, e1-e4. doi:10.1016/j.jmmm.2007.02.009

Singh, H., Du, J., Singh, P., and Yi, T. H. (2018a). Extracellular Synthesis of Silver Nanoparticles by Pseudomonas Sp. THG-LS1.4 and Their Antimicrobial Application. J. Pharm. Anal. 8, 258-264. doi:10.1016/j.jpha.2018.04.004

Singh, J., Dutta, T., Kim, K.-H., Rawat, M., Samddar, P., and Kumar, P. (2018b). 'Green' Synthesis of Metals and Their Oxide Nanoparticles: Applications for Environmental Remediation. J. Nanobiotechnol 16, 84. doi:10.1186/s12951018-0408-4

Singh, P., Kim, Y.-J., Zhang, D., and Yang, D.-C. (2016c). Biological Synthesis of Nanoparticles from Plants and Microorganisms. Trends Biotechnol. 34, 588-599. doi:10.1016/j.tibtech.2016.02.006

Singh, P., Kim, Y. J., Singh, H., Mathiyalagan, R., Wang, C., and Yang, D. C. (2015). Biosynthesis of Anisotropic Silver Nanoparticles byBhargavaea Indicaand Their Synergistic Effect with Antibiotics against Pathogenic Microorganisms. J. Nanomater. 2015, 1-10. doi:10.1155/2015/234741

Singh, P., Kim, Y. J., Wang, C., Mathiyalagan, R., and Yang, D. C. (2016a). Weissella Oryzae DC6-facilitated green Synthesis of Silver Nanoparticles and Their Antimicrobial Potential. Artif. Cell Nanomedicine, Biotechnol. 44, 1569-1575. doi:10.3109/21691401.2015.1064937

Singh, P., Kim, Y. J., and Yang, D. C. (2016b). A Strategic Approach for Rapid Synthesis of Gold and Silver Nanoparticles byPanax Ginsengleaves. Artif. Cell Nanomedicine, Biotechnol. 44, 1949-1957. doi:10.3109/21691401.2015.1115410

Singh, P., Singh, H., Kim, Y. J., Mathiyalagan, R., Wang, C., and Yang, D. C. (2016d). Extracellular Synthesis of Silver and Gold Nanoparticles by Sporosarcina Koreensis DC4 and Their Biological Applications. Enzyme Microb. Technology 86, 75-83. doi:10.1016/j.enzmictec.2016.02.005

Singh, R., and Lillard, J. W. (2009). Nanoparticle-based Targeted Drug Delivery. Exp. Mol. Pathol. 86, 215-223. doi:10.1016/j.yexmp.2008.12.004
Song, J. Y., and Kim, B. S. (2009). Rapid Biological Synthesis of Silver Nanoparticles Using Plant Leaf Extracts. Bioproc. Biosyst. Eng. 32, 79-84. doi:10.1007/s00449008-0224-6

Srinivasan, R., Vigneshwari, L., Rajavel, T., Durgadevi, R., Kannappan, A., Balamurugan, K., et al. (2018). Biogenic Synthesis of Silver Nanoparticles Using Piper Betle Aqueous Extract and Evaluation of its Anti-Quorum Sensing and Antibiofilm Potential against Uropathogens with Cytotoxic Effects: An In Vitro and In Vivo Approach. Environ. Sci. Pollut. Res. 25, 10538-10554. doi:10.1007/s11356-017-1049-0

Sujitha, M. V., and Kannan, S. (2013). Green Synthesis of Gold Nanoparticles Using Citrus Fruits (Citrus limon, Citrus Reticulata and Citrus Sinensis) Aqueous Extract and its Characterization. Spectrochimica Acta Part A: Mol. Biomol. Spectrosc. 102, 15-23. doi:10.1016/j.saa.2012.09.042

Sundrarajan, M., and Gowri, S. (2011). Green synthesis of titanium dioxide nanoparticles by nyctanthes arbor-tristis leaves extract. Chalcogenide Lett. 8 , $447-451$.

Suresh, Y., Annapurna, S., Singh, A. K., Chetana, A., Pasha, C., and Bhikshamaiah, G. (2016). Characterization and Evaluation of Anti-biofilm Effect of green Synthesized Copper Nanoparticles. Mater. Today Proc. 3, 1678-1685. doi:10.1016/j.matpr.2016.04.059

Suryavanshi, P., Pandit, R., Gade, A., Derita, M., Zachino, S., and Rai, M. (2017). Colletotrichum sp.- Mediated Synthesis of sulphur and Aluminium Oxide Nanoparticles and its In Vitro Activity against Selected Food-Borne Pathogens. LWT - Food Sci. Technology 81, 188-194. doi:10.1016/j.lwt.2017.03.038

Swamy, V. S., and Prasad, R. A. M. (2015). Green Synthesis of Silver Nanoparticles from the Leaf Extract of Santalum Album and its Antimicrobial Activity. J. Pharm. Sci. Res. 4, 53-59.

Tang, S., and Zheng, J. (2018). Antibacterial Activity of Silver Nanoparticles: Structural Effects. Adv. Healthc. Mater. 7, 1701503. doi:10.1002/ adhm. 201701503

Thakkar, K. N., Mhatre, S. S., and Parikh, R. Y. (2010). Biological Synthesis of Metallic Nanoparticles. Nanomedicine: Nanotechnology, Biol. Med. 6, 257-262. doi:10.1016/j.nano.2009.07.002

Thukkaram, M., Sitaram, S., Kannaiyan, S. K., and Subbiahdoss, G. (2014). Antibacterial Efficacy of Iron-Oxide Nanoparticles against Biofilms on Different Biomaterial Surfaces. Int. J. Biomater. 2014, 1-6. doi:10.1155/2014/ 716080

Tits, J., Cammue, B. P. A., and Thevissen, K. (2020). Combination Therapy to Treat Fungal Biofilm-Based Infections. Ijms 21, 8873. doi:10.3390/ ijms 21228873

Tripathi, A., Liu, S., Singh, P. K., Kumar, N., Pandey, A. C., Tripathi, D. K., et al. (2017). Differential Phytotoxic Responses of Silver Nitrate (AgNO 3 ) and Silver Nanoparticle (AgNps) in Cucumis Sativus L. Plant Gene 11, 255-264. doi:10.1016/j.plgene.2017.07.005

Uppuluri, P., Srinivasan, A., Ramasubramanian, A., and Lopez-Ribot, J. L. (2011). Effects of Fluconazole, Amphotericin B, and Caspofungin on Candida Albicans Biofilms under Conditions of Flow and on Biofilm Dispersion. Antimicrob. Agents Chemother. 55, 3591-3593. doi:10.1128/AAC.01701-10

Vandecandelaere, I., and Coenye, T. (2015). Microbial Composition and Antibiotic Resistance of Biofilms Recovered from Endotracheal Tubes of Mechanically Ventilated Patients. Adv. Exp. Med. Biol., 137-155. doi:10.1007/978-3-31911038-7_9

Vega-Jiménez, A. L., R. Vázquez-Olmos, A., Antonio Álvarez-Pérez, M., and Antonio Álvarez-Pérez, M. (2019). In vitroAntimicrobial Activity Evaluation of Metal Oxide Nanoparticles. In Nanoemulsions - Properties, Fabrications and Applications, 1-18. doi:10.5772/intechopen.84369

Velgosova, O., Mudra, E., and Vojtko, M. (2021). Preparing, Characterization and Anti-biofilm Activity of Polymer Fibers Doped by Green Synthesized AgNPs. Polymers 13, 605. doi:10.3390/polym13040605

Velsankar, K., R.M, A. K., R, P., V, M., and Sudhahar, S. (2020). Green Synthesis of $\mathrm{CuO}$ Nanoparticles via Allium Sativum Extract and its Characterizations on Antimicrobial, Antioxidant, Antilarvicidal Activities. J. Environ. Chem. Eng. 8, 104123. doi:10.1016/j.jece.2020.104123

Vestby, L. K., Grønseth, T., Simm, R., and Nesse, L. L. (2020). Bacterial Biofilm and its Role in the Pathogenesis of Disease. Antibiotics 9, 59. doi:10.3390/ antibiotics9020059

Vijayakumar, S., Malaikozhundan, B., Saravanakumar, K., Durán-Lara, E. F., Wang, M.-H., and Vaseeharan, B. (2019). Garlic Clove Extract Assisted 
Silver Nanoparticle - Antibacterial, Antibiofilm, Antihelminthic, Antiinflammatory, Anticancer and Ecotoxicity Assessment. J. Photochem. Photobiol. B: Biol. 198, 111558. doi:10.1016/j.jphotobiol.2019.111558

Vijayakumar, S., Vaseeharan, B., Malaikozhundan, B., Gopi, N., Ekambaram, P., Pachaiappan, R., et al. (2017). Therapeutic Effects of Gold Nanoparticles Synthesized Using Musa Paradisiaca Peel Extract against Multiple Antibiotic Resistant Enterococcus faecalis Biofilms and Human Lung Cancer Cells (A549). Microb. Pathogenesis 102, 173-183. doi:10.1016/j.micpath.2016.11.029

Vijayakumar, S., Vinoj, G., Malaikozhundan, B., Shanthi, S., and Vaseeharan, B. (2015). Plectranthus Amboinicus Leaf Extract Mediated Synthesis of Zinc Oxide Nanoparticles and its Control of Methicillin Resistant Staphylococcus aureus Biofilm and Blood Sucking Mosquito Larvae. Spectrochimica Acta Part A: Mol. Biomol. Spectrosc. 137, 886-891. doi:10.1016/j.saa.2014.08.064

Vijayaraghavan, K., and Ashokkumar, T. (2017). Plant-mediated Biosynthesis of Metallic Nanoparticles: A Review of Literature, Factors Affecting Synthesis, Characterization Techniques and Applications. J. Environ. Chem. Eng. 5, 4866-4883. doi:10.1016/j.jece.2017.09.026

Vinotha, V., Iswarya, A., Thaya, R., Govindarajan, M., Alharbi, N. S., Kadaikunnan, S., et al. (2019). Synthesis of $\mathrm{ZnO}$ Nanoparticles Using Insulin-Rich Leaf Extract: Anti-diabetic, Antibiofilm and Anti-oxidant Properties. J. Photochem. Photobiol. B: Biol. 197, 111541. doi:10.1016/ j.jphotobiol.2019.111541

Viola, G. M., and Darouiche, R. O. (2011). Cardiovascular Implantable Device Infections. Curr. Infect. Dis. Rep. 13, 333-342. doi:10.1007/s11908-011-0187-7

von Rosenvinge, E. C., O’May, G. A., Macfarlane, S., Macfarlane, G. T., and Shirtliff, M. E. (2013). Microbial Biofilms and Gastrointestinal Diseases. Pathog. Dis. 67, 25-38. doi:10.1111/2049-632X.12020

Wang, L., Hu, C., and Shao, L. (2017). The Antimicrobial Activity of Nanoparticles: Present Situation and Prospects for the Future. Ijn 12, 1227-1249. doi:10.2147/ IJN.S121956

Wang, W.-N., Tarafdar, J. C., and Biswas, P. (2013). Nanoparticle Synthesis and Delivery by an Aerosol Route for Watermelon Plant Foliar Uptake. J. Nanopart Res. 15, 1413-1417. doi:10.1007/s11051-013-1417-8

Watters, C., Fleming, D., Bishop, D., and Rumbaugh, K. P. (2016). Host Responses to Biofilm. Prog. Mol. Biol. Transl. Sci. 142, 193-239. doi:10.1016/ bs.pmbts.2016.05.007

Xavier, R. J., and Podolsky, D. K. (2007). Unravelling the Pathogenesis of Inflammatory Bowel Disease. Nature 448, 427-434. doi:10.1038/nature06005
Yadi, M., Mostafavi, E., Saleh, B., Davaran, S., Aliyeva, I., Khalilov, R., et al. (2018) Current Developments in green Synthesis of Metallic Nanoparticles Using Plant Extracts: a Review. Artif. Cell Nanomedicine, Biotechnol. 46, S336-S343. doi:10.1080/21691401.2018.1492931

Yin, W., Wang, Y., Liu, L., and He, J. (2019). Biofilms: The Microbial "Protective Clothing" in Extreme Environments. Ijms 20, 3423. doi:10.3390/ijms20143423

Zhang, M., Zhang, K., De Gusseme, B., Verstraete, W., and Field, R. (2014). The Antibacterial and Anti-biofouling Performance of Biogenic Silver Nanoparticles byLactobacillus Fermentum. Biofouling 30, 347-357. doi:10.1080/08927014.2013.873419

Zhang, X., Yan, S., Tyagi, R. D., and Surampalli, R. Y. (2011). Synthesis of Nanoparticles by Microorganisms and Their Application in Enhancing Microbiological Reaction Rates. Chemosphere 82, 489-494. doi:10.1016/j.chemosphere.2010.10.023

Zhu, Y., Wu, J., Chen, M., Liu, X., Xiong, Y., Wang, Y., et al. (2019). Recent Advances in the Biotoxicity of Metal Oxide Nanoparticles: Impacts on Plants, Animals and Microorganisms. Chemosphere 237, 124403. doi:10.1016/j.chemosphere.2019.124403

Zubair, M., Husain, F. M., Qais, F. A., Alam, P., Ahmad, I., Albalawi, T., et al (2021). Bio-fabrication of Titanium Oxide Nanoparticles from Ochradenus Arabicus to Obliterate Biofilms of Drug-Resistant Staphylococcus aureus and Pseudomonas aeruginosa Isolated from Diabetic Foot Infections. Appl. Nanosci. 11, 375-387. doi:10.1007/s13204-020-01630-5

Conflict of Interest: The authors declare that the research was conducted in the absence of any commercial or financial relationships that could be construed as a potential conflict of interest.

Publisher's Note: All claims expressed in this article are solely those of the authors and do not necessarily represent those of their affiliated organizations, or those of the publisher, the editors and the reviewers. Any product that may be evaluated in this article, or claim that may be made by its manufacturer, is not guaranteed or endorsed by the publisher.

Copyright $\odot 2021$ Das, Ghosh and Nayak. This is an open-access article distributed under the terms of the Creative Commons Attribution License (CC BY). The use, distribution or reproduction in other forums is permitted, provided the original author(s) and the copyright owner(s) are credited and that the original publication in this journal is cited, in accordance with accepted academic practice. No use, distribution or reproduction is permitted which does not comply with these terms. 